Aus der Abteilung Allgemeinmedizin

(Prof. Dr. med. M. M. Kochen, MPH, FRCGP)

im Zentrum Innere Medizin

der Medizinischen Fakultät der Universität Göttingen

\title{
Die Arzt-Patient-Beziehung in der ambulanten Schizophreniebehandlung und ihre Bedeutung für die Compliance aus Sicht der Patienten
}

\author{
Eine qualitative Untersuchung
}

\author{
Inaugural-Dissertation \\ zur Erlangung des Doktorgrades \\ der Medizinischen Fakultät \\ der Georg-August-Universität zu Göttingen
}

vorgelegt von

Anke Tenbieg

aus Hamburg

Göttingen 2007 
Dekan: Prof. Dr. med. C. Frömmel

1. Berichterstatter: Prof. Dr. disc. pol. W. Himmel

2. Berichterstatter/in:

3. Berichterstatter/in:

Tag der mündlichen Prüfung: 


\section{Inhaltsverzeichnis}

\begin{tabular}{lll}
\hline 1 & Einleitung & 9
\end{tabular}

2 Theoretischer Hintergrund und Stand der Forschung 10

2.1 Definition und Diagnosekriterien der Schizophrenie . . . . . . . . . . . . . . . 10

2.1 .1 Definition Schizophrenie . . . . . . . . . . . . . . . . . . . . . 10

2.1 .2 Diagnosekriterien der Schizophrenie . . . . . . . . . . . . . . . . . 11

2.2 Die ambulante Versorgungssituation schizophrener Patienten . . . . . . . . . 13

2.2 .1 Die Betreuung durch den Hausarzt . . . . . . . . . . . . . . . . . . 13

2.2 .2 Die kombinierte Betreuung durch Haus- und Facharzt . . . . . . . . . 15

2.2 .3 Die ambulante Versorgungssituation aus Sicht der Patienten . . . . . 16

2.3 Schizophrenie und Compliance . . . . . . . . . . . . . . . . . . . . . . . . . 19

2.3 .1 Definition Compliance . . . . . . . . . . . . . . . . . . . . . . . . 19

2.3.2 Klinische Relevanz von Compliance und Noncompliance . . . . . . . 20

2.3 .3 Determinanten der Medikamentencompliance. . . . . . . . . . . . . . 20

2.3.4 Die Bedeutung der Arzt-Patient-Beziehung für die Compliance . . . . 21

2.3 .5 Compliance aus Sicht der Patienten . . . . . . . . . . . . . . 22

\begin{tabular}{|lll}
\hline 3 & Fragestellung und Ziel & 23
\end{tabular}

4 Material und Methoden 24

4.1 Studiendesign des Rahmenprojekts . . . . . . . . . . . . . . . . . . . . . . 24

4.2 Methodische Anlage der Untersuchung . . . . . . . . . . . . . . . . 25

4.3 Teilnehmende Praxen und Patienten . . . . . . . . . . . . . . . . . . . . 26

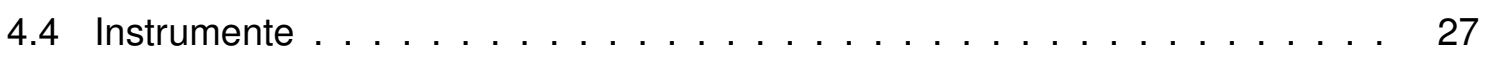

4.4 .1 Das qualitative Interview . . . . . . . . . . . . . . . . . . . 27

4.4 .2 Entwicklung des Leitfadens . . . . . . . . . . . . . . . . . . . 28

4.4 .3 Die Critical Incident Technique . . . . . . . . . . . . . . . . . . . . . . 29

4.5 Durchführung der Untersuchung $\ldots \ldots \ldots \ldots$. . . . . . . . . . . . . . 30

4.5 .1 Durchführung der Interviews . . . . . . . . . . . . . . . . . . 30

4.5 .2 Datenerfassung . . . . . . . . . . . . . . . . . . . . . . . . . . . . . . . . . . . . . .

4.6 Datenanalyse . . . . . . . . . . . . . . . . . . . . . . . . . . 31

4.6 .1 Materialorientiertes Erfassen von Themen . . . . . . . . . . . . . . . . 32

4.6 .2 Erstellung eines Kodierleitfadens (Codebook) . . . . . . . . . . . . . 33 
4.6 .3 Kodierung der Interviews . . . . . . . . . . . . . . . 33

$4.6 .4 \quad$ Erfassen von Inhalten und Beziehungen . . . . . . . . . . . . . 34

4.7 Datenschutz und Patientenaufklärung . . . . . . . . . . . . . . . . 35

5 Ergebnisse 36

5.1 Beschreibung der Stichprobe $\ldots \ldots \ldots \ldots \ldots \ldots \ldots$

5.2 Zuständigkeit des Hausarztes $\ldots \ldots \ldots \ldots . . \ldots \ldots$

5.2 .1 Der Hausarzt als Gatekeeper . . . . . . . . . . . . . . . . . . 38

5.2 .2 Der Hausarzt als Körperarzt . . . . . . . . . . . . . . . . . . . . . . 39

5.2.3 Weiterführen der Pharmakotherapie durch den Hausarzt. . . . . . . 40

5.2 .4 Der Hausarzt als Ansprechpartner für psychische Probleme. . . . . . 40

5.3 Zuständigkeit des Facharztes . . . . . . . . . . . . . . . . 41

5.3.1 Der Facharzt als Spezialist für die psychische Erkrankung . . . . . . . 41

5.3.2 Der Facharzt als Ansprechpartner für Probleme des Alltags . . . . . . 43

5.4 Einflussfaktoren auf Compliance . . . . . . . . . . . . . . 44

5.4.1 Persönliche Einstellung gegenüber Psychopharmaka . . . . . . . 44

5.4 .2 Erfahrungen mit Medikamenten und Compliance . . . . . . . . . 46

5.4 .3 Auswirkungen von aktuell auftretenden Nebenwirkungen . . . . . 49

5.4 .4 Informationen über Medikamente . . . . . . . . . . . . . . . . 50

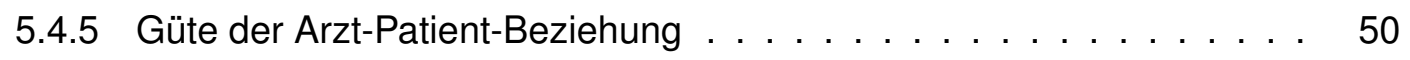

5.4 .6 Therapeutische Entscheidungen . . . . . . . . . . . . . 51

5.4 .7 Umgang mit Noncompliance in der therapeutischen Beziehung . . . . 53

5.4 .8 Ärztliche Faktoren . . . . . . . . . . . . . . . . . . . . . 55

5.4 .9 Äußere Einflüsse . . . . . . . . . . . . . . . . . . . . . . . 56

5.5 Einflussfaktoren einer guten Arzt-Patient-Beziehung . . . . . . . . 56

5.5 .1 Dauer und Kontakthäufigkeit der Beziehung . . . . . . . . . . 57

5.5 .2 Infrastruktur . . . . . . . . . . . . . . . . . . 59

5.5 .3 Persönlichkeit und Kompetenz des Arztes . . . . . . . . . . 60

5.5 .4 Autonomie . . . . . . . . . . . . . . . . 63

5.5 .5 Arzt-Patient-Passung und Kommunikation . . . . . . . . . . 63

5.5 .6 Soziales Umfeld . . . . . . . . . . . . . . . . . . . . 65

6 Diskussion 68

6.1 Die Rolle des Hausarztes in der ambulanten Schizophreniebehandlung . . . 68

6.2 Ressourcen und Defizite von Hausarzt und Facharzt . . . . . . . . . . . 72

6.3 Begünstigende Faktoren für eine tragfähige therapeutische Beziehung aus Sicht der Patienten . . . . . . . . . . . . . . . . . . . . 74

6.4 Auswirkung der Arzt-Patient-Beziehung auf die Compliance . . . . . . . 76

6.5 Persönliche Erfahrungen und Einstellungen als weitere Einflussfaktoren von 
6.6 Kritische Bilanz der methodischen Umsetzung . . . . . . . . . . . . . . 80

6.7 Schlussfolgerung . . . . . . . . . . . . . . . . . . . 81

\begin{tabular}{lll}
\hline 7 & Zusammenfassung & 83
\end{tabular}

\begin{tabular}{lll}
\hline 8 & Literaturverzeichnis & 85
\end{tabular}

\begin{tabular}{ll}
\hline Anhänge & 93
\end{tabular}

A.1 Informed Consent . . . . . . . . . . . . . . . . . . . . . . . . . . . . . . . . . 94

A.2 Patientendaten . . . . . . . . . . . . . . . . . . . . . . . . 95

A.3 Leitfaden Patienteninterview $\ldots \ldots$. . . . . . . . . . . . . . . . . . . . 96

A.4 Leitfaden Patienteninterview I $\ldots \ldots \ldots$. . . . . . . . . . . . . . 97

A.5 Codebook . . . . . . . . . . . . . . . . . . . . . . . . . . . . . 98

A.6 Definitonen der Codes . . . . . . . . . . . . . . . . . . . . . . . . . . . . . 99

A.7 Diagramm Codegruppen . . . . . . . . . . . . . . . . . . 100 


\section{Abbildungsverzeichnis}

4.1 Schematische Darstellung der zirkulären Strategie . . . . . . . . . . . . . . 26

$5.1 \quad$ Einflussfaktoren auf Compliance $\ldots \ldots \ldots \ldots$. . . . . . . . . . 45

6.1 Die Rolle des Hausarztes in der ambulanten Schizophreniebehandlung . . . 71

6.2 Beteiligung am therapeutischen Entscheidungsprozess . . . . . . . . . . 77 


\section{Tabellenverzeichnis}

2.1 Gegenüberstellung der Diagnosekriterien der Schizophrenie nach ICD-10 und DSM-IV . . . . . . . . . . . . . . . . . . . . 12

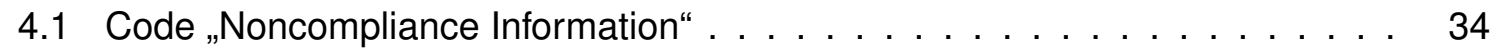

5.1 Patientenstichprobe $\ldots \ldots \ldots \ldots \ldots \ldots$ 


\section{Vorbemerkung}

In der folgenden Arbeit wird aufgrund der besseren Lesbarkeit vorwiegend die männliche Sprachform verwendet. Soweit nicht ausdrücklich anders hervorgehoben, sind hiermit beide Geschlechter gleichermaßen gemeint.

Der Begriff Hausarzt fasst praktische Ärzte, Fachärzte für Allgemeinmedizin und hausärztlich tätige Internisten zusammen. Der Begriff Facharzt bzw. Spezialist wird in Abgrenzung hierzu für Psychiater und Nervenärzte sowie Ärzte unterschiedlicher Fachrichtungen mit therapeutischer Zusatzausbildung benutzt. 


\section{Einleitung}

Die Schizophrenie ist eine psychische Erkrankung, die in der modernen Medizin spätestens seit Prägung des Begriffs durch Eugen Bleuler einen festen Platz hat. Von dieser schweren Erkrankung, deren Ursachen bis heute nicht vollständig geklärt sind, sind etwa $1 \%$ der Bevölkerung betroffen. Das sind alleine in Deutschland ca. 800.000 Erkrankte, ca. 10-15\% der Patienten beenden ihr Leben durch Suizid. Die Behandlung der Schizophrenie ist dauerhaft notwendig und erfordert in der Regel mindestens zeitweise eine fachärztliche Therapie. Dennoch ist auch ein Großteil der Hausärzte in die Betreuung schizophrener Patienten involviert, da die meisten von ihnen einen Hausarzt haben. Ein häufiges Problem in der Betreuung dieser Patienten ist - vor allem krankheitsbedingt - die Noncompliance. Erschwerend kommt hinzu, dass auch die Ausbildung vertrauensvoller (therapeutischer) Beziehungen erheblich beeinträchtigt ist.

In der Vergangenheit wurde die ambulante Schizophreniebehandlung überwiegend aus der Sicht der Ärzte beleuchtet. Gleiches gilt für die Complianceforschung auf diesem Gebiet. Sie war darauf ausgelegt, Ärzten Wissen und Werkzeuge an die Hand zu geben, um die Compliance zu verbessern. Diese Arbeit befasst sich mit der Thematik aus Sicht der Patienten. Sie möchte einen Einblick in die (subjektiv erlebte) ambulante Versorgungssituation schizophrener Patienten bieten und hofft, eine neue Perspektive auf das Thema zu eröffnen.

Stark beeinflusst ist die Studie durch die Idee des „Kompetenznetzes Schizophrenie“: Neben einer horizontalen Vernetzung der Forschungseinrichtungen soll vor allem auch durch eine vertikale Vernetzung der Forschungszentren mit Einrichtungen der Routineversorgung eine Verbesserung der Behandlung erreicht werden. Diese Arbeit ist innerhalb des mittlerweile abgeschlossenen Projekts „Optimierung der Schizophreniebehandlung in der Allgemeinarztpraxis“ des vom Bundesminister für Bildung und Forschung geförderten „Kompetenznetzes Schizophrenie" entstanden. 


\section{Theoretischer Hintergrund und Stand der Forschung}

Der Entwicklung des Studiendesigns und der Entscheidung über angewandte Methoden war eine eingehende theoretische Orientierung über den aktuellen Forschungsstand zum Thema der Arbeit vorgeschaltet. Hierbei wurde auf Literatur zurückgegriffen, die einerseits über das interne Suchsystem der Medizinischen Bibliothek, andererseits über allgemeine Suchmaschinen (http://www.google.de) und fachspezifische Literatur-Datenbanken (vornehmlich Medline; vgl. http://www.pubmed.com) im Internet ausfindig gemacht werden konnte. Als Suchbegriffe wurden unter anderem Schlüsselwörter wie „Schizophrenie“, „schizophrenia“, „treatment refusal“, „patient compliance“, „Arzt-Patient-Beziehung“, „physician patient relation“, „antipsychotic agents“, „psychotic disorders“, sowie ihre Kombinationen untereinander und mit „Hausarzt“ und „primary health care“ bzw. „family practice“ verwandt. Auch zur Erarbeitung einer angemessenen Methodik wurde über das Internet mit Suchbegriffen wie „qualitative Forschung“, „qualitative research“, „qualitative Methoden“, „qualitative methods“ u. ä. auf Literatur zugegriffen.

\subsection{Definition und Diagnosekriterien der Schizophrenie}

\subsubsection{Definition Schizophrenie}

Historisch betrachtet (MACHLEIDT 1999) wurde der Begriff Schizophrenie (griechisch: schízein = spalten; phrén = Zwerchfell, Geist, Gemüt) erstmals 1911 von dem Psychiater Eugen Bleuler (1857-1939) verwandt. Die Erscheinungsbilder der Erkrankung waren allerdings bereits vorher bekannt und von Emil Kraeplin (1856-1926) im Jahre 1898 unter dem Terminus „Dementia praecox" zusammengefasst. Noch heute bilden die Arbeiten dieser beiden Autoren zusammen mit dem Krankheitskonzept nach Kurt Schneider (1887-1967) den Grundstein einer modernen Definition des Begriffes „Schizophrenie“, die man in Anlehnung an die AMERICAN PSYCHIATRIC ASSOCIATION (1994) und SCHMIDT JU (2004) wie folgt zusammenfassen kann:

Schizophrenie beschreibt eine Gruppe von Krankheitsbildern, die sich den funktionellen (endogenen) Psychosen zuordnen lassen. Es tritt eine Beeinträchtigung von Fühlen, Denken, Wahrnehmen und Handeln auf, ohne dass dafür ein körperliches Korrelat gefunden werden kann. Charakteristisch ist eine erhöhte Durchlässigkeit der Ich-Umwelt-Grenzen und 
eine Desintegration der psychischen Elementarfunktionen, die mit Affektstörungen und sozialem Rückzug einhergehen. Typisch sind weiterhin Sinnestäuschungen und Wahnwahrnehmungen. Der Betroffene erlebt eine mit der äußeren Realität nicht mehr in Übereinstimmung zu bringende innere Wirklichkeit im Sinne einer doppelten Buchführung.

\subsubsection{Diagnosekriterien der Schizophrenie}

Die Diagnose „Schizophrenie“, im ICD-10 als F20 codiert (DILLING et al. 2004), orientiert sich an dem Vorhandensein klar umschriebener Symptome über einen bestimmten Zeitraum. Diese Merkmale werden in folgenden Gruppen zusammengefasst:

1. Gedankenlautwerden, Gedankeneingebung oder Gedankenentzug, Gedankenausbreitung

2. Kontrollwahn, Beeinflussungswahn, Gefühl des Gemachten, Wahnwahrnehmung, anhaltender, kulturell unangemessener und völlig unrealistischer Wahn von der eigenen Superexistenz

3. Kommentierende oder dialogische Stimmen

4. Anhaltende Halluzinationen jeder Sinnesmodalität, begleitet von Wahngedanken oder überwertigen Ideen

5. Gedankenabreißen oder Einschiebungen in den Gedankenfluss, was zu Zerfahrenheit, Danebenreden oder Neologismen führt

6. Katatone Symptome wie Erregung, Haltungsstereotypien oder Flexibilitas cerea (wächserne Biegsamkeit), Negativismus, Mutismus und Stupor

7. „Negative“ Symptome wie auffällige Apathie, Sprachverarmung, verflachte oder inadäquate Affekte mit sozialem Rückzug und Nachlassen der sozialen Leistungsfähigkeit.

Erforderlich für die Diagnose „Schizophrenie“ ist mindestens ein eindeutiges Symptom (zwei oder mehr, wenn weniger eindeutig) der oben genannten Gruppen 1-4 oder mindestens zwei Symptome der Gruppen 5-7. Diese Symptome müssen fast ständig während eines Monats oder länger deutlich vorhanden gewesen sein. Bei Auftreten der beschriebenen Symptome über einen kürzeren Zeitraum (behandelt oder unbehandelt) kann dagegen lediglich eine „akute schizophreniforme psychotische Störung“ (F23.2) diagnostiziert werden. Retrospektiv kann möglicherweise eine Prodromalphase identifiziert werden, die bereits Wochen oder Monate vor dem Auftreten psychotischer Symptome beginnen kann, jedoch keine diagnostische Wertigkeit besitzt. Eine Schizophrenie soll nicht bei ausgeprägten manischen oder depressiven Symptomen diagnostiziert werden, wenn diesen nicht schizophrene Symptome vorausgegangen sind. Genauso wenig zutreffend ist diese Diagnose bei eindeutiger Gehirnerkrankung, während einer Intoxikation oder während des Entzugs. 
Neben dem ICD-10 besteht als zweites operationalisiertes Diagnosesystem das Diagnostic and Statistical Manual of Mental Disorders (DSM-IV) (AMERICAN PSYCHIATRIC ASSOCIATION 1994), dessen Regelsystem trotz Unterschieden im Detail in großen Bereichen mit dem ICD-10 (DILLING et al. 2004) überlappt (Tabelle 2.1).

Tabelle 2.1: Gegenüberstellung der Diagnosekriterien der Schizophrenie nach ICD-10 und DSM-IV

\begin{tabular}{|c|c|}
\hline ICD-10 & DSM-IV \\
\hline $\begin{array}{l}\text { Kontrollwahn, Beeinflussungswahn, } \\
\text { Wahnwahrnehmung }\end{array}$ & Wahn, besonders bizarrer Wahn \\
\hline $\begin{array}{l}\text { Halluzinationen, besonders } \\
\text { kommentierende oder dialogisierende } \\
\text { Stimmen }\end{array}$ & $\begin{array}{l}\text { Stimmungsinkongruente Halluzinationen, } \\
\text { besonders kommentierende oder } \\
\text { dialogisierende Stimmen }\end{array}$ \\
\hline $\begin{array}{l}\text { Gedankenlautwerden, Gedankenentzug, } \\
\text { Gedankeneingebung, } \\
\text { Beeinflussungserlebnisse, Zerfahrenheit, } \\
\text { Gedankenabreißen u. a. }\end{array}$ & Zerfahrenheit \\
\hline Katatone Symptome & Katatone Symptome \\
\hline $\begin{array}{l}\text { Negative Symptome wie Apathie, } \\
\text { Sprachverarmung, verflachter Affekt }\end{array}$ & $\begin{array}{l}\text { Affektarmut, Antriebsmangel, sozialer } \\
\text { Rückzug }\end{array}$ \\
\hline & Verschlechterung der sozialen Adaptation \\
\hline $\begin{array}{l}\text { Charakteristische Symptomatik } \\
\text { mindestens } 1 \text { Monat }\end{array}$ & $\begin{array}{l}\text { Kontinuierliche Anzeichen der Erkrankung } \\
\text { mindestens } 6 \text { Monate }\end{array}$ \\
\hline Keine nachweisbare organische Ursache & Keine nachweisbare organische Ursache \\
\hline Keine nachweisliche Intoxikation & Keine nachweisliche Intoxikation \\
\hline
\end{tabular}

Klinisch wird die Schizophrenie traditionell noch in mehrere Subtypen unterschieden. So stehen bei dem paranoid-halluzinatorischen Typ Wahn und Halluzinationen im Vordergrund, während der hebephrene Typ, der fast ausschließlich im Jugendalter auftritt, von affektiven Störungen mit unreflektiert bedenkenloser Grundstimmung, formalen Denkstörungen sowie einem allgemein enthemmten Sozialverhalten bestimmt ist. Die anderen Symptome können hier weitestgehend fehlen bzw. in schwacher Ausprägung vorliegen. Typisch für die katatone Schizophrenie sind psychomotorische Störungen, die zwischen extremer Hyperkinese und Stupor schwanken, weiterhin treten Befehlsautomatismen, Negativismen, Haltungsstereotypien etc. auf. Bei dieser Form besteht die Gefahr des Übergangs in eine lebensbedrohliche perniziöse Katatonie mit Stupor, Hyperthermie und anderen vegetativen Entgleisungen. 
Beim Residualtyp prägt eine Persönlichkeitsveränderung im Sinne von Antriebsmangel, Affektarmut und sozialem Rückzug (Minussymptomatik) das Bild. Bei der Schizophrenia simplex wird dieses Residualsyndrom ohne vorausgegangene Phasen mit Positivsymptomatik (Wahn, Halluzinationen) erreicht (MöLLER et al. 1996, S. 139 f.).

\subsection{Die ambulante Versorgungssituation schizophrener Patienten}

Eine an Schizophrenie erkrankte Person benötigt dauerhaft und regelmäßig medizinische Betreuung. Im Rahmen eines mehrdimensionalen Konzeptes beinhaltet die Therapie der Erkrankung neben der Pharmakotherapie, die primär Aufgabe des Arztes ist, auch eine Soziound Psychotherapie.

In der Richtlinie Schizophrenie der Arbeitsgemeinschaft der Wissenschaftlichen Medizinischen Fachgesellschaften (AWMF) sollen gemäß der Deutschen Gesellschaft für Psychiatrie, Psychotherapie und Nervenheilkunde (DGPPN) in der medikamentösen Therapie standardmäßig typische und atypische Neuroleptika eingesetzt werden, die bei Bedarf durch anxiolytisch und sedativ wirkende Benzodiazepine ergänzt werden (DGPPN 2005). Die starken unerwünschten Wirkungen insbesondere der Neuroleptika, die eine genaue Einstellung auf die individuelle Optimaldosis notwendig machen, erfordern einen regelmäßigen Kontakt zwischen Arzt und Patient, um Fehldosierungen so früh zu erkennen, dass keine bleibenden Schäden in Form von Spätdyskinesien auftreten. Dieselbe Richtlinie sieht als Behandlungsinstitutionen in der akuten Episode neben stationären Einrichtungen auch Tageskliniken, Ambulanzen / Polikliniken und Praxen niedergelassener Fachärzte vor. Für die Versorgung im Intervall sind dann neben anderen ambulanten Einrichtungen auch explizit Allgemeinarztpraxen aufgeführt (DGPPN 2005).

\subsubsection{Die Betreuung durch den Hausarzt}

Obwohl die Spezifität der Betreuung nahe legt, dass die Patienten zumindest bezüglich ihrer psychischen Erkrankung von einem Psychiater oder Nervenarzt betreut werden, trifft dies in der Dauertherapie nicht unbedingt zu. Nach einer Studie in Deutschland, in der HAASEN und STARK (1997) 77 Patienten in stationärer Behandlung über ihre ambulante Arztwahl befragten, sucht immerhin ein Drittel (31\%) für die Behandlung der psychiatrischen Erkrankung ausschließlich den Hausarzt auf. Korrespondierend gaben 66,1\% der befragten Allgemeinärzte an, zwischen einem und 30 schizophrene Patienten zu betreuen (im Mittel 3,3 Patienten pro Arzt). Lediglich 33,9\% betreuten demnach keine schizophrenen Patienten (HAASEN et al. 1993). Diese Daten beruhen auf einer retrospektiven Befragung (mittels Fragebogen und / oder telefonischer Befragung) von 594 Allgemeinärzten in Hamburg.

Die Zahlen zeigen die Bedeutung des Hausarztes in der Betreuung schizophrener Patien- 
ten, wobei sich, wie oben beschrieben, die akute Episode der Schizophrenie in der Regel der Betreuung durch den Allgemeinarzt entzieht. In den Aufgabenbereich des Hausarztes fällt hingegen die Intervention zu Beginn eines akuten Schubs der Erkrankung, die - abhängig von dem Schweregrad des Verlaufs - aus einer Anpassung bereits bestehender Medikation oder der Zuweisung zur stationären bzw. fachärztlichen Behandlung besteht. Diese Aufgaben werden also auch in der ambulanten Betreuung schizophrener Patienten erfüllt, wobei die AWMF-Richtlinie die Allgemeinarztpraxis in der Remissionsphase explizit als Behandlungsinstitution aufführt (DGPPN 2005). Außerdem ist der Hausarzt auch oftmals der erste Arzt, der durch seinen regelmäßigen Kontakt zum Patienten die Symptome der psychischen Erkrankung sieht oder von innen berichtet bekommt. Ihm kommt daher auch eine wichtige Rolle in der Früherkennung der Schizophrenie zu (CARR 1997). Somit wird der Hausarzt zum Bindeglied zwischen dem Patienten und den psychiatrischen Versorgungseinrichtungen (SPIESSL und CORDING 1999), zumal seine primäre Therapie und sein Überweisungsverhalten Einfluss auf den weiteren Verlauf der Erkrankung nehmen. In dieser Phase der Vermittlung in fachärztliche Betreuung profitiert der Hausarzt von einem „Vertrauenskredit“ (EICH 1990, S. 228), den er bei den Patienten und - als Familienarzt - oft auch bei dessen Angehörigen genießt. Außerdem hat er in der Regel bessere Kenntnis über die lokalen, sozialen und familiären Ressourcen als seine Kollegen aus speziellen Fachgebieten. Dies gibt inm die Chance, Langzeitentwicklungen des Patienten in dessen spezifischem Milieu wahrzunehmen und zu beeinflussen (BÖKER 1988). Gerade wegen dieser Vertrautheit mit dem Patienten ist der Hausarzt oftmals die erste Anlaufstation auch und gerade für psychische Probleme, insbesondere für diejenigen Patienten, die eine Stigmatisierung im Zusammenhang mit ihrer Erkrankung befürchten (SPIESSL und CORDING 2000). Es liegt nun an ihm, die fachärztliche Behandlung individuell und vertrauensvoll einzuleiten (HAASEN und STARK 1997). Die rechtzeitige Überweisung an den Facharzt und die Vorbereitung auf die dort bevorstehende Behandlung ist auch ein wesentlicher Bestandteil der Erwartungen, mit denen Patienten ihren Hausarzt aufsuchen (KLINGENBERG et al. 1996). Elne solche zeitnahe Überweisung an den Fachkollegen erfolgt insbesondere dann, wenn er für den Allgemeinarzt im Bezug auf Erreichbartkeit und Verfügbarkeit von Terminen gut zugängig ist (CHRISTENSEN et al. 1989).

Neben diesen Aspekten der Betreuung psychischer Erkrankung übernimmt der Hausarzt aber selbstverständlich auch die Behandlung somatischer Krankheiten, die unabhängig oder aber als Begleiterkrankung der Schizophrenie (z. B. komorbide Sucht, unerwünschte Arzneimittelwirkungen etc.) auftreten können (HAASEN et al. 1993). Vor allem wegen seiner Vertrautheit mit Patient und Angehörigen wird der Hausarzt immer stärker bei der Entwicklung therapeutischer Konzepte in Kooperation mit niedergelassenen Fachkollegen sowie psychiatrischen Kliniken eingebunden. Dabei „sollte der behandelnde Arzt seine Kompetenzen ausschöpfen, jedoch auch seine eigenen Grenzen kennen, sich vor Überengagement schützen und die ausreichende Abgrenzung auf der persönlichen Ebene sicherstellen" (EICH 1990, S. 231). Gemäß der DGPPN (1997) besteht sollte der Hausarzt psychische Störungen Erken- 
nen, die Patienten bereaten und begleiten sowie zum Teil auch primär behandeln (z. B. bei depressiven Störungen). Außerdem besteht seine Aufgabe im rechtzeitigen Weiterleiten an den Facharzt bei diagnostischer Unklarheit oder Therapieresistenz und die Rückübernahme und Weiterbehandlung bei erfolgter Diagnostik und indizierter Langzeitbehandlung.

Neben den Untersuchungen zur Bedeutung der Hausärzte in der Betreuung psychiatrischer und speziell schizophrener Patienten in Deutschland zeigten auch internationale Studien den hohen Stellenwert der Allgemeinärzte bei der Versorgung dieser Patienten. So schätzten Hausärzte in einer amerikanischen Befragung, dass durchschnittlich $22,6 \%$ ihrer erwachsenen Patienten unter signifikanten emotionalen und psychiatrischen Problemen litten, die Aufklärung und Behandlung rechtfertigen (ORLEANS et al. 1985). Ausgewertet wurden 350 Fragebögen (Rücklauf 57\%) von Hausärzten mit der Fachbezeichnung „Family Practitioner"einer landesweiten amerikanischen Stichprobe. In der Befragung von 1.005 Patienten einer großen, amerikanischen Praxis, die überwiegend Einwohner einer Großstadt mit niedrigem sozioökonomischem Status und Bildungsniveau betreute, fanden OLFSEN et al. (2002) sogar eine Prävalenz von 30,7\% für psychische Störungen. Sie identifizierten diese mit Hilfe des Patient Health Questionnaire for the Primary Care Evaluation of Mental Disorders (DSM-IV Version). Jeder fünfte der von innen befragten Patienten $(20,9 \%)$ gab an, in kürzerer Vergangenheit eines oder mehrere der erfragten psychotischen Symptome erlebt zu haben.

\subsubsection{Die kombinierte Betreuung durch Haus- und Facharzt}

Wie bereits erwähnt, legt die spezifische Therapie der Schizophrenie, die klassischerweise der Psychiatrie zugeordnet wird, nahe, dass die Patienten durch einen entsprechenden Facharzt wie etwa Psychiater oder Nervenarzt zumindest mitbetreut werden. Dies bestätigten auch Hausärzte bei einer Befragung in Hamburg für 69,0\% der von ihnen betreuten Schizophrenen (HAASEN et al. 1993). Es ist genau diese Gruppe, die laut einer weiteren Studie von HAASEN und STARK (1997) die beste ärztliche Betreuung erfährt. Als Qualitätskriterium legten sie einen errechneten Quotienten aus der Dauer seit der Ersthospitalisation und der Zahl der stationären Aufenthalte zugrunde, der letztendlich die durchschnittliche Zeit in Jahren zwischen Hospitalisationen darstellt. Hier sei jedoch anzumerken, dass die Zahlen durch den erheblichen Anteil an erstmals hospitalisierten Patienten (36\%) an Aussagekraft verlieren, da es zumeist diese Patienten sind, die ausschließlich durch den Hausarzt betreut werden. Außerdem wird nicht zwischen unterschiedlichen Schweregraden der Erkrankung differenziert, welcher bei der Wahl zwischen Hausarzt oder Facharzt durchaus von Bedeutung sein könnte.

Mit der Kooperation zwischen Hausärzten und psychiatrischen Facheinrichtungen beschäftigten sich SPIESSL und CORDING (1999). Sie gehen hierbei davon aus, dass Allgemeinärzte und Nervenärzte zu gleichen Teilen psychiatrische Leistungen im Rahmen der kassenärztlichen Versorgung erbringen und berufen sich auf eine bayrische Studie, nach 
der Hausärzte sogar mehr psychisch Kranke behandeln als Nervenärzte, zu einem nicht unerheblichen Teil (25\% der Patienten) gänzlich ohne psychiatrische Konsultation (MELLER et al. 1989). Auch sie ordnen dem Hausarzt dabei eine Funktion im Sinne des Gatekeepers zu (vgl. Abschnitt 2.2.1.

Das Konzept vom Hausarzt als Gatekeeper (engl. für Torhüter) geht davon aus, dass er in der Regel die erste Anlaufstelle bei gesundheitlichen Problemen ist und daher mit einem breiten Spektrum von Patienten und Erkrankungen in Kontakt kommt. Hieraus ergibt sich die Aufgabe, „banale“ von schwerwiegenden Krankheiten zu unterscheiden und die Patienten entsprechend in die notwendige - ambulante oder stationäre - fachspezifische Behandlung weiterzuleiten (ABHOLZ und KOCHEN 2006).

In dieser wichtigen Schlüsselposition erfahren Allgemeinärzte jedoch häufig sowohl fachlich als auch zeitlich ihre Grenzen. So geben $80 \%$ der Hausärzte an, zu wenig Zeit für die Behandlung psychisch Kranker zu haben, und $90 \%$ wünschen sich in der Betreuung dieser Patienten die Zusammenarbeit mit einem Facharzt (FALLOON et al. 1996). Hierbei legen sie insbesondere Wert auf eine rasche, offene und persönliche Kommunikation mit dem Fachkollegen, schnelle Zusendung der entsprechenden Unterlagen (z. B. Entlassungsbrief) sowie eine klare Diagnose mit konkreten Therapievorschlägen (SPIESSL und CORDING 1999). Die Kommunikation scheint also das zentrale Thema in der Zusammenarbeit zu sein und wird in 43\% aller Aussagen von niedergelassenen Nervenärzten / Psychiatern und Allgemeinärzten angesprochen (SPIESSL und CORDING 2000). Zudem steht der Zugang, insbesondere die Wartezeiten bis zur Konsultation, wiederum im direkten Zusammenhang zur Kommunikation zwischen Hausarzt und Facharzt (MONALTO et al. 1994). Dementsprechend wurden auch als häufigste Gründe für Unzufriedenheit in der Zusammenarbeit mangelnder Informationsfluss (verzögerte Übermittlung der Arztbriefe und fehlende telefonische Erreichbarkeit des Ansprechpartners) und unzureichendes Feedback angegeben (SPIESSL und CORDING 2000).

\subsubsection{Die ambulante Versorgungssituation aus Sicht der Patienten}

Setzt man sich mit der Versorgungssituation für ein spezielles Patientenkollektiv auseinander, so ist es nahe liegend, die Thematik auch aus dessen Sicht zu betrachten, insbesondere die Ansprüche, Erwartungen und Zufriedenheit sowie die Determinanten, die diese Aspekte bestimmen. Dies ist zwar in einer Reihe von Studien für primärmedizinisch relevante Patientengruppen erfolgt, eine spezielle Betrachtung schizophrener Patienten fehlt jedoch weitgehend. Sie ist aber für die Ziele des „Kompetenznetzes Schizophrenie“ durchaus interessant.

\section{Erwartungen an die hausärztliche Versorgung}

In einer groß angelegten Studie (EUROPEP = European Project on Patient Evaluation of General Practice Care) beschäftigten sich Wissenschaftler aus mehreren europäischen Län- 
dern zunächst mit den Erwartungen der Patienten an den Hausarzt und dann mit der Beurteilung der Hausärzte und ihrer Praxen durch die Patienten. Anhand eines selbständig auszufüllenden Fragebogens wurden Patienten $(n=3540)$ zu Aspekten der hausärztlichen Versorgung und deren Gewichtung befragt. Aus diesen Daten konnten GROL et al. (1999) eine Rangliste der zehn wichtigsten Erwartungen, die die Patienten an ihren Hausarzt hatten, zusammenstellen. Ein Hausarzt sollte:

1. genügend Zeit zum Zuhören, Reden und Erklären haben

2. im Notfall schnell zur Verfügung stehen

3. alle Informationen über seine Patienten vertraulich behandeln

4. den Patienten alles sagen, was sie über ihre Krankheit wissen wollen

5. es den Patienten ermöglichen, offen über Probleme zu reden

6. auch kurzfristig einen Termin vergeben

7. sich regelmäßig fortbilden, um auf dem neuesten Stand zu bleiben

8. nicht nur Krankheiten heilen, sondern auch Prävention betreiben

9. den Nutzen von Medikamenten und ärztlichem Rat kritisch abwägen

10. den Grund für Untersuchungen und Behandlungen detailliert erklären.

Die große Bedeutung dieser Aspekte findet sich auch in einer englischen Studie (OGDEN et al. 1997): Zwar sahen sowohl Patienten als auch Ärzte die Behandlung von Erkrankungen als Hauptaufgabe des Arztes, aber auch Aspekte des zwischenmenschlichen Umganges spielten eine bedeutende Rolle. So gehörte es für $64,3 \%$ der Patienten zur Rolle des Arztes, „ein Freund zu sein“. Allgemeiner betrachtet zeigten SHERBOURNE et al. (1999), dass Gesundheit aus der Sicht der Patienten nicht nur die körperliche, sondern auch die seelische und soziale Gesundheit mit einschließt. Folglich müsste auch der Arzt, diese Aspekte von Gesundheit in die Betreuung seiner Patienten einbeziehen.

Sucht man nun nach Erwartungen, die speziell von schizophrenen Patienten an ihre Hausärzte herangetragen werden, stößt man in der Literatur lediglich auf die Gründe, weshalb der Arzt aufgesucht wurde, und auf eine knappe Bewertung des Kontakts. So gaben 59\% der schizophrenen Patienten als Hauptgrund für den Besuch ihres Hausarztes somatische Beschwerden an, nur 22\% kamen, weil es innen psychisch schlechter ging, 15\%, weil sie ein Rezept für Psychopharmaka brauchten (HAASEN und STARK 1997). Weder aus diesen Motiven für den Arztbesuch, noch aus der Einschätzung der Zufriedenheit mit dem Arzt kann man jedoch auf die konkreten Erwartungen, die diese Patienten an ihren Arzt haben, Rückschlüsse ziehen. 


\section{Bewertung der hausärztlichen Versorgung}

Die Bewertung der ärztlichen Versorgung durch die Patienten hat in den letzten Jahren insbesondere unter Aspekten der Qualitätssicherung an Bedeutung gewonnen. Wenn die Zufriedenheit der Patienten auch kein objektives Maß für die medizinischen Bedürfnisse und die Qualität der Versorgung ist (SCHMITTDIEL et al. 1997), so ist sie als subjektive Wahrnehmung jedoch im Hinblick auf eine tragfähige therapeutische Beziehung mit allen ihren positiven Konsequenzen (WILLIAMS 1994) nicht zu unterschätzen. Die Exploration ihrer Determinanten kann also helfen, Schwächen in der Versorgung aufzuzeigen (KLINGENBERG et al. 1996).

Eben diese Bewertung war Inhalt des zweiten Teils der bereits erwähnten EUROPEPStudie. Erwartungsgemäß wurden sehr hohe Werte bei der allgemeinen Zufriedenheit mit dem Hausarzt erzielt. So sahen 95,4\% der Befragten keinen Grund für einen Arztwechsel (KLINGENBERG et al. 1999). Dies bestätigt auch die recht hohe allgemeine Zufriedenheit, eines großen amerikanischen Patientenkollektivs einer kalifornischen Health Maintenance Organization ( $n=10.205$ ). Mittels Fragebogen bewerteten $76,9 \%$ der Patienten, die ihren Arzt frei gewählt hatten, ihre Zufriedenheit mit „exzellent" oder „sehr gut“ (SCHMITTDIEL et al. 1997). Einzelne Aspekte der Versorgung schnitten aber durchaus unterschiedlich ab. Vergleichsweise gute Bewertung erhielten die vertrauliche Behandlung der Patientendaten (73,4\% „ausgezeichnet“) und das Zuhören und die Gründlichkeit des Arztes (65,1\%). Weit seltener hingegen wurden die Einbeziehung der Befragten in medizinische Entscheidungen $(38,3 \%)$ als „ausgezeichnet“bewertet, ebenso die Erinnerung des Arztes an frühere Gespräche $(23,1 \%)$. Häufig unbeantwortet blieb die Frage nach der Vorbereitung auf einen Facharztbesuch oder Krankenhausaufenthalt: 29,5\% der Befragten gaben hier keine Antwort. Von denen, die antworteten, fühlten sich 53,3\% „ausgezeichnet“ vorbereitet. Die Bewertung „schlecht“ (unteres Ende der Skala) wurde insgesamt sehr wenig vergeben, am häufigsten für die Wartezeiten mit 2,6\%.

Betrachtet man die Bewertung des Arztes nun wiederum aus der Sicht schizophrener Patienten, fehlt hier eine ähnlich differenzierte Aufschlüsselung der einzelnen Aspekte, wie sie oben für das Gesamtkollektiv primärärztlicher Patienten beschrieben ist. HAASEN und STARK (1997) konnten lediglich zeigen, dass Patienten, die ausschließlich von ihrem Hausarzt betreut wurden, mit diesem deutlich zufriedener waren als diejenigen, die von Hausarzt und Facharzt versorgt wurden. Auf einer Skala von 1 („gar nicht zufrieden“) bis 7 („,sehr zufrieden“) vergaben sie die Note 5,9 im Gegensatz zu 4,8 bei Doppelbetreuung. Am schlechtesten schnitten die Nervenärzte ab (Note 3,4), bei denen die Patienten in ausschließlicher Betreuung waren. Dieses Ergebnis könnte jedoch durch den wahrscheinlich unterschiedlichen Schweregrad der Erkrankung verzerrt sein, da vermutlich die ausschließlich hausärztlich betreuten Patienten weniger stark belastet waren. 


\subsection{Schizophrenie und Compliance}

\subsubsection{Definition Compliance}

Das englische Wort Compliance bedeutet in der wörtlichen Übersetzung „Erfüllung“, „Befolgung“ oder „Einhaltung“. Im medizinisch-therapeutischen Kontext definierten HAYNES et al. (1982, S. 12) den Begriff vor dem Hintergrund einer eher autoritär-hierarchisch geprägten Arzt-Patient-Beziehung in den 80er Jahren als:

"... den Grad , in dem das Verhalten einer Person in Bezug auf die Einnahme eines Medikamentes, das Befolgen einer Diät oder die Veränderung des Lebensstils mit dem ärztlichen oder gesundheitlichen Rates korrespondiert. Auch der Begriff „konsequentes Befolgen“ könnte gleichbedeutend an die Stelle des Begriffs „Compliance“ treten."

Der moderne Compliance-Begriff hingegen wird eher als Bereitschaft des Patienten definiert, eine Behandlung einzugehen und aktiv mitzutragen. Sie ist somit das Einverständnis mit therapeutischen Interventionen (ALBUS 1995). Parallel wird häufig der Begiff Adherence (deutsch: Adhärenz) verwendet. Die World Health Organization (WHO) hebt in Ihrer Definition hervor, dass - in Abgrenzung zu Compliance - das Einverständnis des Patienten mit dem ärztlichen Rat als Grundvoraussetzung gegeben sein muss, um überhaupt von Adhärenz sprechen zu können. Sie sehen sowohl den Arzt als auch den Patienten als aktive Partner in einer therapeutischen Beziehung. Auf dieser Basis bezeichnet der Begriff Adherence das Ausmaß, in dem das Verhalten eines Patienten bei der Medikamenteneinnhame mit den Empfehlungen des Arztes korrespondiert (WHO 2003). In der Literatur findet man häufig beide Begriffe synonym verwendet. Dies gilt auch im Weiteren für diese Arbeit. $\mathrm{PE}-$ TERMANN (1998, S. 74) hebt noch hervor, dass es sich bei Compliance nicht um ein stabiles Persönlichkeitsmerkmal handele, vielmehr sei es ein komplexes, situationsabhängiges und dynamisches Phänomen, welches im Behandlungsverlauf variabel sei. Dementsprechend gibt es eine Vielzahl von Faktoren, die Einfluss auf die Compliance haben. Hierzu gehören Krankheitsmerkmale (z. B. Misstrauen im Rahmen der paranoiden Psychose), Persönlichkeitsmerkmale des Patienten, Art der Therapie (z. B. komplizierte Therapiepläne, Einsatz von Hilfsmitteln für das Einhalten des Therapieplans), Wirkungen und Nebenwirkungen der Therapie, die Arzt-Patient-Beziehung, psychosoziale Faktoren (MARDER 2003, BUCHANAN 1992), sowie eine möglicherweise bestehende comorbide Alkohol- oder Drogenabhängigkeit (KASHNER et al. 1991). Neben diesen genannten faktoren hebt BEBBINGTON (1995) noch hervor, dass auch der Verlust von sekundärem Krankheitsgewinn bei erfolgreicher Therapie zur Noncompliance führen kann.

Wie oben dargestellt, bezieht sich Compliance auf alle Aspekte einer Behandlung, welche neben der medikamentösen Therapie auch Psychotherapie, Physiotherapie, Diät etc. enthalten kann. In dieser Untersuchung wird das Augenmerk jedoch primär auf die Medikamenten- 
Compliance und die Aufrechterhaltung eines regelmäßigen therapeutischen Kontakts zwischen Arzt und Patient gerichtet sein.

\subsubsection{Klinische Relevanz von Compliance und Noncompliance}

Noncompliance ist einer der Hauptfaktoren, die den Verlauf der Schizophrenie negativ beeinflussen (FERNANDO et al. 1990, CASPAR und REGAN 1993, SCHOOLER 2003). Allein dies ist Grund genug, sich mit der Medikamentencompliance zu befassen. Darüber hinaus hat Noncompliance auch aus ökonomoscher Sicht negative Folgen.

Mehrere Studien haben gezeigt, dass die Medikamentencompliance im Allgemeinen eher gering ist. SACKETT und SNOW (1979) gehen von einer durchschnittlichen Compliancerate von $50 \%$ bei Langzeittherapien unterschiedlicher Erkrankungen aus. Ein aktuellerer Review, der sich mit dieser Thematik innerhalb der Forschung der letzten 50 Jahre befasst, kommt auf eine Non-Adherence-Rate von 25-40\% (DIMATTEO 2004). Auch bei schizophrenen Patienten liegt der Wert innerhalb dieses weitgefassten Bereichs. FENTON et al. (1997) errechneten bei einer Metaanalyse aus 15 verschiedenen Studien eine mittlere Noncompliancerate von $55 \%$ bei Schizophrenen, wobei sowohl Patienten mit oraler als auch Patienten mit Depot-Neuroleptikatherapie in die Untersuchung einbezogen, jedoch nicht differenziert wurden. Dies deckt sich auch mit dem Ergebnis eines aktuelleren Literatur-Reviews (LACRO et al. 2002), in dem die Noncompliance-Rate schizophrener Patienten im Bezug auf ihre antipsychotische Therapie bei $40-50 \%$ lag. Für den Krankheitsverlauf ist jedoch nicht alleine die Tatsache, ob ein Wirkstoff zugeführt wird oder nicht, entscheidend, sondern Compliance an sich - also als Verhalten - ist ein beeinflussender Faktor. EPSTEIN (1984) wertete mehrere placebokontrollierte Studien vor diesem Hintergrund aus und konnte nicht nur zeigen, dass diejenigen, die das Verum erhielten, bessere Therapieergebnisse als die Placebo- Gruppe erzielten. Interessanterweise zeigte auch die therapietreue Placebo-Gruppe deutlich bessere Ergebnisse als die Gruppen von Patienten, die sich nicht an den Therapieplan hielten, unabhängig von Wirkstoff oder Placebo. Dieser Effekt ließ sich bei der Analyse von insgesamt fünf Studien mit unterschiedlichen Erkrankungen und medikamentösen Therapien bestätigen, was als Hinweis auf ein allgemeines Phänomen gedeutet werden kann.

\subsubsection{Determinanten der Medikamentencompliance}

Vielfältige Aspekte können Einfluss auf die Compliance haben. FENTON et al. (1997) teilten diese in verschiedene Gruppen ein:

- patientenabhängige Faktoren, z. B. soziodemographische Merkmale

- krankheitsabhängige Faktoren, z. B. die Krankengeschichte, der Schweregrad und der Subtyp der Erkrankung sowie krankheitsbedingte Einschränkung von Kognition und Erinnerung und die Krankheitseinsicht 
- medikamentenabhängige Faktoren, z. B. unerwünschte Wirkungen oder die Komplexität des Therapieplans

- Umweltfaktoren, z. B. die familiäre Unterstützung oder praktische Hindernisse (z. B. ein weiter Weg zum Arzt)

- Beziehungsaspekte, z. B. ein gutes Vertrauensverhältnis zwischen Arzt und Patient oder schlechte Erfahrungen und Misserfolge in der Behandlung

- Komorbidität, insbesondere Suchterkrankungen wie Alkoholismus oder anderer Drogenabusus (KASHNER et al. 1991).

Besondere Aufmerksamkeit kommt in dieser Arbeit der Arzt-Patient-Beziehung zu. Sie ist eine wichtige Säule jeder Therapie, welche im Idealfall als ein gemeinsames Projekt von Arzt und Patient verstanden wird.

\subsubsection{Die Bedeutung der Arzt-Patient-Beziehung für die Compliance}

Der Einfluss der Arzt-Patient-Beziehung auf die Compliance war schon Inhalt einer Reihe von Studien, die alle die Bedeutung der Art der Beziehung für das Erreichen guter Adherence zeigen. So weist BEBBINGTON (1995) in seiner Abhandlung über Inhalt und Kontext von Compliance darauf hin, dass ein vertrauensvolles Verhältnis dem Arzt bessere Überzeugungsmöglichkeit bietet. Wichtig sei auch, den Patienten in seiner Persönlichkeit ernst zu nehmen, um die Asymmetrie der therapeutischen Beziehung zu verringern und die Therapie zu einem gemeinsamen Anliegen zu machen. In einer Befragung von 370 Patienten in Hausarztpraxen nahmen diejenigen, die Vertrauen und Übereinstimmung mit ihrem Hausarzt als gut bezeichneten, mit etwa 30\% höherer Wahrscheinlichkeit die verordneten Medikamente auch ein (NGAIRE et al. 2004). Bereits 20 Jahre früher zeigten NELSON et al. (1975), dass der einzig herausragende Einflussfaktor auf Compliance die Wahrnehmung der Patienten war, ob der Arzt an innen als Personen interessiert war oder nicht. Einen direkten Zusammenhang zwischen der therapeutischen Beziehung und der Compliance zeigte eine amerikanische Studie über die Arzt-Patient-Beziehungen von 143 nicht-chronisch schizophrenen Patienten (FRANK und GUNDERSON 1990). Die Autoren richteten ihre Aufmerksamkeit dabei auf den Beginn und Aufbau der Beziehungen. Sie konnten einerseits zeigen, dass nur eine Minderheit schizophrener Patienten gute therapeutische Beziehungen aufbaut, dass aber genau diese Minderheit mit deutlich erhöhter Wahrscheinlichkeit in der Therapie verblieb, bessere Medikamentencompliance zeigte und einen insgesamt besseren Verlauf der Erkrankung erzielte. Um so beunruhigender ist es, dass KLINGENBERG et al. (1999) in einer Patientenbefragung im Rahmen der EUROPEP-Studie herausfanden, dass $64,7 \%$ der Patienten in hausärztlicher Behandlung sich nicht immer ganz sicher waren, wie wichtig die Befolgung der ärztlichen Ratschläge tatsächlich sei. Gründe für diese Unsicherheit von Seiten der Patienten wurden leider nicht weitergehend erfragt. 


\subsubsection{Compliance aus Sicht der Patienten}

In der Mehrzahl der Studien wurde das Thema Compliance aus der Sicht der Ärzte untersucht, thematisiert wurde zum Beispiel ihr Umgang mit Noncompliance (HESZEN-KLEMENS 1987) und ihre Möglichkeiten, eine bessere Compliance der Patienten zu erreichen (HAEBERLIN THOMAS et al. 1992, FENTON et al. 1997, HORNUNG et al. 1998). Die Perspektive der Patienten fand jedoch insbesondere in der aktuellen Forschung wenig bis keine Beachtung. Die Entwicklung des „Health-Belief-Model of Compliance" etwa lässt sich schon bis ins Jahr 1975 zurückverfolgen (BECKER und MAINMAN 1975). Es setzt sich mit der Entscheidungsfindung des Patienten bezüglich Compliance oder Noncompliance und mit den Faktoren, die den Prozess beeinflussen, auseinander. Noch konsequenter aus der Patientenperspektive befasste sich STIMSON (1974) mit dem Thema: Ausgehend von einem hierarchischen Modell, das der Forschung über Compliance zugrunde läge („der Arzt gibt Anweisungen und der Patient befolgt sie"), stellte er hingegen den Patienten in den Mittelpunkt und warf folgende Fragen auf: Was erwartet der Patient? Was tut er, um seine Erwartungen erfült zu sehen? Werden seine Erwartungen erfüllt? Wie nehmen beide Seiten Einfluss auf den Verlauf der Interaktion? Auf diese Fragen lassen sich bis heute in der Literatur keine klaren Antworten finden, insbesondere nicht für psychisch kranke Menschen und speziell nicht für schizophrene Patienten.

Alle hier zum Thema Compliance vorgestellten Studien unterstreichen die Bedeutung einer tragfähigen Arzt-Patient-Beziehung für eine erfolgreiche Therapie. Es ist daher sinnvoll, sich mit den Determinanten für eine solche Beziehung weiter auseinanderzusetzen. 


\section{Fragestellung und Ziel}

Neben niedergelassenen Fachärzten für Psychiatrie und Nervenheilkunde sind Hausärzte wesentlich an der ambulanten Betreuung schizophrener Patienten beteiligt. Da wenig darüber bekannt ist, welche Aufgabenschwerpunkte, Stärken und Schwächen ihnen diese Patienten zuschreiben, untersucht die vorliegende Arbeit aus Patientensicht, welche Rolle der Hausarzt - in Abgrenzung zum Facharzt - in der ambulanten Schizophreniebehandlung einnimmt. Da die Medikamentencompliance bei antipsychotischer Medikation den Verlauf der Erkrankung (FERNANDO et al. 1990, CASPAR und REGAN 1993) und die Häufigkeit der Rehospitalisationen (SCHOOLER 2003) erheblich beeinflusst und wesentlich durch eine vertrauensvolle therapeutische Beziehung mit angemessenem Informationsfluss gefördert wird (GAEBEL 1998, FENTON et al. 1997, BEBBINGTON 1995), soll es in dieser Arbeit auch um Faktoren gehen, die vor allem bei an Schizophrenie erkrankten Menschen zur Ausbildung einer guten Arzt-Patient-Beziehung beitragen. Im Einzelnen geht es daher um folgende Fragestellungen:

- Welche Rolle nimmt der Hausarzt im Vergleich zum Facharzt in der ambulanten Schizophreniebehandlung ein und welche Merkmale der hausärztlichen bzw. fachärztlichen ambulanten Betreuung werden durch schizophrene Patienten als Stärken und Schwächen wahrgenommen?

- Welche Aspekte empfinden die Patienten in ihrer aktuellen ambulanten Betreuung als förderlich für die Medikamentencompliance und die Güte der Arzt-Patient-Beziehung?

- Wie kann aus Sicht der Patienten eine bessere Arzt-Patient-Beziehung und damit einhergehend eine bessere Compliance erreicht werden?

Ziel der Arbeit ist die Aufdeckung spezifischer Ressourcen und Defizite von Hausarzt und Facharzt in der ambulanten Schizophreniebehandlung aus Sicht der Patienten. Dies schließt auch die Frage nach bestehenden und möglichen Aufgabenbereichen des jeweiligen Arztes in der ambulanten Schizophreniebehandlung mit ein. Auf der Basis dieser Ergebnisse könnten in weiterführenden Schritten Interventionen erarbeitet werden, um die Betreuung der Patienten längerfristig zu optimieren. 


\section{Material und Methoden}

\subsection{Studiendesign des Rahmenprojekts}

Diese Arbeit war in ein Forschungsvorhaben des Kompetenznetzes Schizophrenie (siehe auch: http://kompetenznetz-schizophrenie.de) eingebettet. Ein Teilprojekt dieses Forschungsvorhabens - die „Optimierung der Schizophreniebehandlung in der Allgemeinarztpraxis" - wurde in der Abteilung Allgemeinmedizin der Universität Göttingen durchgeführt. Das Kompetenznetz Schizophrenie ist ein bundesweiter Forschungsverbund, der vom Bundesministerium für Bildung und Forschung gefördert wurde. Ziel des Netzes ist es, Diagnostik, Prävention, Akut- und Langzeittherapie sowie Rehabilitation schizophrener Störungen zu optimieren. Dies soll durch gezielte, praxisnahe Forschungsprojekte geschehen, die vom Netzwerkgedanken unterstützt werden, welcher eine horizontale und vertikale Vernetzung zwischen Forschungs- und Versorgungseinrichtungen durch den Auf- und Ausbau von Kommunikations- und Kooperationsstrukturen beinhaltet. Neben der in dieser Arbeit behandelten Fragestellung geht es in dem Teilprojekt „Optimierung der Schizophreniebehandlung in der Allgemeinarztpraxis“ auch um die Erwartungen, Wünsche und Anliegen der Angehörigen schizophrener Patienten an die hausärztliche Versorgung.

In Hildesheim und Kassel wurde im Jahr 2000 bereits eine Vorerhebung durchgeführt, bei der sämtliche Fachärzte für Allgemeinmedizin, praktische Ärzte, hausärztlich tätige Internisten und Ärzte ohne Gebietsbezeichnung einen Fragebogen zu folgenden Themen erhielten: Anzahl der betreuten schizophrenen Patienten, Neuzugänge, Kontakthäufigkeit zwischen Arzt und schizophrenem Patient, Schwierigkeiten in der Behandlung, bestehende Hilfsangebote / Kooperationen und Einschätzung ihrer Effektivität, Einschätzung der eigenen Kompetenz und Teilnahmebereitschaft an der eigentlichen Untersuchung. In Kassel wurden 149 Bögen an 18 Ärzte ohne Gebietsbezeichnung, 78 Allgemeinärzte, 43 Internisten und 10 praktische Ärzte versandt. Der Rücklauf betrug lediglich 22,82\% (34 Fragebögen). Bei 74 in Hildesheim angeschriebenen Ärzten (3 Ärzte ohne Gebietsbezeichnung, 44 Allgemeinärzte, 20 Internisten, 7 praktische Ärzte) betrug die Rücklaufquote 32,43\% (24 Bögen), so dass insgesamt 58 verwertbare Fragebögen vorlagen.

In der Rekrutierungsphase für diese Untersuchung wurde die angeschriebene Gruppe von Hausärzten auf die Landkreise Hildesheim und Kassel erweitert. Außerdem wurden niedergelassene Fachärzte für Psychiatrie und Nervenheilkunde sowie die in den Ambulanzen der psychiatrischen Universitätsklinik und des Landeskrankenhauses Göttingen tätigen Ärzte kontaktiert. Es wurde zusätzlich versucht, die Teilnahmebereitschaft zu steigern, indem 
im telefonischen Kontakt die Studie noch einmal kurz vorgestellt und mögliche Fragen der Ärzte beantwortet wurden.

Um Patienten und Angehörige zur Teilnahme an der Studie zu gewinnen, wurden - neben den oben erwähnten Hausärzten - auch Mitglieder einer Selbsthilfegruppe für Psychoseerfahrene in Gießen direkt angesprochen. Weiterhin konnten auch über den Kontakt zu einer Selbsthilfegruppe für Angehörige Patienten für das Projekt gewonnen werden. So sollte ein möglichst breites Spektrum an Patienten und deren Angehörigen einbezogen werden.

\subsection{Methodische Anlage der Untersuchung}

Nach der erwähnten Vorerhebung (Abschnitt 4.1) musste davon ausgegangen werden, dass schizophrene Patienten - entgegen der Literatur - kaum ausschließlich vom Hausarzt betreut, sondern zumeist parallel von einem Facharzt versorgt werden. Schon dabei wurde deutlich, dass elementare Basisdaten über die Versorgungsrealität ambulanter Schizophreniebehandlung fehlten. Daraufhin wurde das Studiendesign modifiziert und die Forschungsziele wurden neu definiert (vgl. Kapitel 3). Da nun die subjektiv wahrgenommene Realität und die Bedürfnisse schizophrener Patienten in der Gesundheitsversorgung in möglichst breiter Streuung erfasst werden sollten, eignete sich ein qualitatives Studiendesign in besonderer Weise (WRIGHT et al. 1998). Die Fragestellung vereint soziale Aspekte (die therapeutische Beziehung und ihre Einflüsse auf die Compliance) und klinische Interessen (Erfolg der Therapie durch Compliance). Dies ist nach BERKWITS und ARONOWITZ (1995) ideal für den Einsatz eines qualitativen Forschungsdesigns.

Der Ansatz ist vor allem deskriptiv, wobei durch eine strukturierte Auswertung und Darstellung der Informationen auch Zusammenhänge hypothetisch angedeutet werden können. Es geht also darum, einen Sachverhalt, der primär unbekannt ist, zu beschreiben und dadurch eine Informationsgrundlage für weitere Schritte (z. B. weitere Forschung mit dem Ziel der Generalisierbarkeit oder der Entwicklung von Interventionen zur Verbesserung der Versorgungssituation) zu schaffen. Für diese Exploration unbekannter Bereiche ist ebenfalls der Einsatz qualitativer Methoden geeignet (FLICK 2000). Um größtmögliche Offenheit zu wahren, wurde eine zirkuläre Forschungsstrategie gewählt (Abbildung 4.1). So wurde der Interviewleitfaden zunächst basierend auf dem in der Literatur verfügbaren Wissen zum Thema erstellt, dann jedoch nach den Erkenntnissen aus den ersten Interviews strukturell und inhaltlich modifiziert, indem neue Aspekte eingearbeitet wurden. Ähnliches gilt auch für den Kodierleitfaden sowie die Datenanalyse angelehnt an MILLER und CRABTREE (1994).

Zusammenfassend kann man also sagen, dass die deskriptive Zielsetzung des Projekts und die sozialen, interaktiven und weitgehend unbekannten Elemente des Forschungsgegenstands eine explorative Offenheit im Forschungsdesign erforderten, wie sie besonders bei einem qualitativen Ansatz mit zirkulärer Forschungsstrategie gegeben ist. 


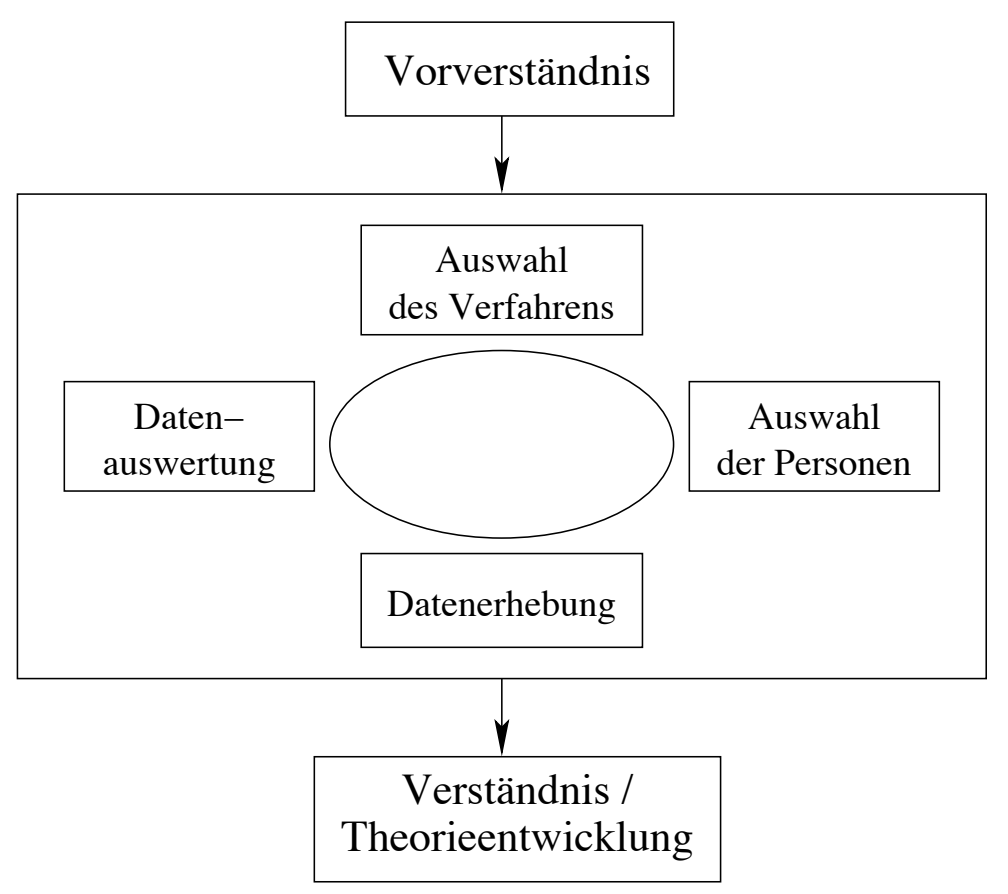

Abbildung 4.1: Schematische Darstellung der zirkulären Strategie

\subsection{Teilnehmende Praxen und Patienten}

Die Rekrutierung schizophrener Patienten als Interviewpartner erfolgte über Hausärzte sowie Selbsthilfegruppen in den Städten Hildesheim, Kassel, Gießen und Göttingen. Die Region wurde so großflächig gewählt, um trotz des geringen Rücklaufs eine ausreichend große Stichprobe zu erhalten. Den schizophrenen Personen wurde von den behandelnden Ärzten oder Therapeuten bzw. in den Selbsthilfegruppen ein Informationsblatt zu der Untersuchung und ein Informed Consent-Bogen (s. Anhang A.1) ausgehändigt. Diesen konnten sie mit Angabe ihrer Telefonnummer direkt an die Abteilung Allgemeinmedizin zurücksenden. Eine Mitarbeiterin des Projekts stellte dann den persönlichen Kontakt her und vereinbarte ein Treffen. Als Aufwandsentschädigung erhielten die Teilnehmer 25 Euro.

Die Rekrutierung erfolgte auch noch parallel zur Datenerhebung und -analyse, bis eine inhaltliche „Sättigung“ des Datenmaterials gegeben war. Die angestrebte Heterogenität der Daten wurde berücksichtigt, indem Patienten zum einen über Hausärzte (8 Interviews), zum anderen über Selbsthilfegruppen für Patienten (8 Interviews) oder Angehörige (4 Interviews) rekrutiert wurden.

Alle teilnehmenden Patienten sollten über 18 Jahre alt sein und bereits eine bekannte Diagnose Schizophrenie oder Psychose aus dem schizophrenen Formenkreis haben, die den Kriterien des ICD-10 (F20) entsprach (DILLING et al. 2004). Vorausgesetzt war außerdem, dass sie den Informed Consent-Bogen selbständig ausfüllen konnten. Ausschlusskriterien für die Teilnahme waren eine akute psychotische Episode, akute Suizidgefährdung, Depres- 
sion und Substanzmissbrauch.

\subsection{Instrumente}

Die Daten wurden jeweils mit einem Fragebogen zur Erfassung personenbezogener Daten und einem Interview erhoben. Der Fragebogen Anhang A.2 sollte in erster Linie dazu dienen, die Stichprobe soziodemographisch zu beschreiben. Weiterhin wurden krankheitsspezifische Informationen (Medikation, Länge der Arzt-Patient-Beziehung und Diagnose) erfragt. Mit dem Interview hingegen wurde das inhaltlich relevante Datenmaterial erhoben. In der Entwicklung des Leitfadens und der Durchführung des Interviews wurde die Critical Incident Technique (vgl. Abschnitt 4.4.3) berücksichtigt.

\subsubsection{Das qualitative Interview}

Als Methode zur Datenerhebung wurde das persönliche Interview gewählt. Im wissenschaftlichen Sinne handelt es sich hierbei um ein planmäßiges Vorgehen mit wissenschaftlicher Zielsetzung, bei dem die „Versuchsperson“ durch eine Reihe gezielter Fragen oder mitgeteilter Stimuli zu verbalen Informationen angeregt werden soll. Zur Anwendung kommt das persönliche Interview in einer frühen Forschungsphase zu einem bestimmten Thema, in der zunächst Hypothesen generiert werden sollen. Geeignet ist das Interview auch zur Aufdeckung komplexer Strukturen im Bezug auf hochgradig subjektive emotional besetzte Gegenoder Umstände geht, in denen auch ein hohes Maß an Ambivalenz auftreten kann $(\mathrm{HOHL}$ 2000, S. 144).

Für diese Untersuchung wurde als spezielle Ausprägung des qualitativen Interviews das semistrukturierte Leitfadeninterview angewandt. Es handelt sich dabei um ein ermittelndes Interview (LAMNEK 1995, S. 38), in dem der Informationsfluss einseitig vom Befragten zum Interviewer gerichtet ist. Differenziert man diese Intention des Interviews weiter, so handelt es sich um ein analytisches Interview nach KOOLWIJK (1974, S. 16). Bei dieser Form des Interviews liegt der Schwerpunkt auf der Erfassung sozialer Sachverhalte und Zusammenhänge. Es erfolgt eine Analyse der Äußerungen im Interview vor dem Hintergrund eines theoretischen Grundwissens über den Sachverhalt. Die Auseinandersetzung mit dem sozialen Phänomen der Arzt-Patient-Beziehung und ihren Determinanten fällt somit in den klassischen Anwendungsbereich dieser Methode.

Der bestimmende Faktor für die Standardisierung der Interviews war der Leitfaden. Er gab einen thematischen Rahmen vor, der einerseits weitestgehend eingehalten werden sollte, andererseits aber so offen gehalten war, dass er den Befragten nicht zu sehr eingeschränkte. Der Befragte sollte dadurch Themenbereiche, die nicht direkt vorgesehen waren, inm aber relevant erschienen, ansprechen können. Zwar ist auch das Leitfadeninterview von der Asymmetrie der Gesprächssituation, die durch die Richtung des Informationsflusses entsteht, geprägt, sie erreicht jedoch nicht das Ausmaß wie in anderen Befragungs- 
formen, z. B. dem standardisierten Interview. Vielmehr entsteht eine Asymmetrie, „wie sie auch für Alltagsgespräche nicht untypisch ist" (LAMNEK 1995, S. 40). Diese möglichst natürliche Situation spiegelt sich auch im Kommunikationsstil des Interviews wider. Es wird ein weicher bis neutraler Stil angestrebt, der von Empathie und einem Vertrauensverhältnis geprägt sein sollte, um so dem Befragten die Möglichkeit zu geben, offen zu berichten. Erst so können verlässliche und gültige Informationen erhoben werden (LAMNEK 1995, S. 57f). Der Interviewer nimmt dabei eine passive Rolle ein und greift lediglich bei Themenwechseln ein (KOOLWIJK 1974, S. 17).

Außerdem wichtig für das Gespräch ist die Sprache, derer sich der Interviewer bedient. Er muss sich zum Ziel setzen, die Sprache seines Gegenübers zu sprechen, damit dieser ihn verstehen und frei aus seiner Lebenswelt berichten kann (HOFFMANN-RIEM 1980). Hier soll also eine Trennung von wissenschaftlicher Auseinandersetzung und Erfassung einer persönlichen Situation erfolgen. Die Verwendung wissenschaftlich theoretischer Begriffe und das direkte Verwenden von Forschungsfragen (z. B. „Was sind die Determinanten der Beziehung zu ihrem Arzt?") verbieten sich daher (HERMANNS 2000, S. 367f).

Zusammenfassend kann man das gewählte Erhebungsinstrument als ein semistrukturiertes, persönliches Leitfadeninterview beschreiben, in dem unter der Verwendung überwiegend offener Fragen ein möglichst weicher Kommunikationsstil angestrebt wurde. In der Interviewsituation sollte eine empathisch vertrauensvolle Atmosphäre geschaffen werden, die es den schizophrenen Patienten ermöglichte, offen und möglichst detailliert über ihre persönlichen Erfahrungen, Meinungen und Wünsche im Umgang mit ihren Ärzten zu berichten.

\subsubsection{Entwicklung des Leitfadens}

Zur Durchführung der Interviews wurde ein Leitfaden entwickelt, der zunächst als Orientierung in der Interviewsituation dienen und dann zu einer besseren Vergleichbarkeit der Interviews bei der Auswertung beitragen sollte (HOHL 2000, S. 146). Der Leitfaden ist das Resultat einer wissenschaftlichen Erarbeitung, die unter Einbeziehung relevanter wissenschaftlicher Literatur zur inhaltlich und systematisch begründeten Fragestellung führt (SCHMIDTGRUNERT 1990, S. 43). Eine erste Version berücksichtigte folgende Elemente des Gesprächsverlaufs und Themenkomplexe (vgl. Anhang A.3):

- kurze erklärende Einleitung

- offener Beginn, in dem der Patient gebeten wurde, sich an herausragende Situationen in der Behandlung zu erinnern und diese zu beschreiben (Daten zur Anwendung der Critical Incident Technique, vgl. Abschnitt 4.4.3

- Betreuungssituation (Wer betreut was, wie häufig?)

- Arzt-Patient-Beziehung 
- Motivation zum Arztbesuch

- Erwartungen

- Zufriedenheit

- abschließende Formalitäten (Patientenvergütung etc.).

Nach der Durchführung einiger Interviews und der Reflektion über erste Erfahrungen mit dem Leitfaden innerhalb der Forschungsgruppe wurden im Sinne der Prozessorientierung (SCHMIDT-GRUNERT 1990, S. 42) einige Änderungen vorgenommen. Formell wurde der Leitfaden verkürzt und weniger konkret gehalten. Damit wurde die von HOPF (2000, S. 358) beschriebene „Leitfadenbürokratie“- die Tendenz den Leitfaden in der Interviewsituation oberflächlich abzuhaken - noch weiter eingedämmt. Außerdem wurde die Bitte an den Patienten, die letzten Arztbesuche zu beschreiben, an das Ende gestellt. In Bezug auf den Inhalt ergaben sich im Prozess der Interviewführung und parallelen Durchsicht der ersten Interviews neue Aspekte, die unter Einbeziehung der entsprechenden Fachliteratur, zur Aufnahme des Themenkomplexes „Medikamente“ führten. Über einige Zwischenschritte ergab sich somit ein Leitfaden Anhang A.4, der wie folgt gegliedert war:

- kurze erklärende Einleitung

- Krankheitsverlauf / Behandlungssituation (weitgehend offener Teil)

- Themenkomplex „therapeutische Beziehung“

- Themenkomplex „Medikamente“

- Zurückliegender Arztbesuch (Critical Incident Technique)

- Abschluss (Patientenvergütung etc.).

An diesem Punkt soll noch einmal betont werden, dass der Leitfaden lediglich als Richtlinie für das Gespräch angesehen wurde. Er diente nicht als starre Vorgabe, die Interviewer und Befragte zu sehr einengen sollte. Die Reihenfolge und Gewichtung der Themen wurden vielmehr von dem natürlichen Gesprächsverlauf vorgegeben.

\subsubsection{Die Critical Incident Technique}

Die Critical Incident Technique (CIT) ist eine Methode in der qualitativen Forschung, die 1954 von John Flanagan entwickelt wurde (FLANAGAN 1954). Sie ist ein Instrument zur systematischen Erfassung und Identifikation von Verhaltensweisen, die in einer spezifischen Situation maßgeblich für den Erfolg oder Misserfolg von Einzelpersonen oder Gruppen sind (EMMUS 1999). Es werden dabei retrospektive Daten erhoben, d. h. die betreffenden Personen werden nach einem Ereignis schriftlich oder im Interview befragt, welche Verhaltensweisen vor, 
während und nach dem Ereignis das Gelingen oder Misslingen beeinflusst haben. Diese Critical Incidents (engl. für „kritische Vorfälle“) können außerdem durch Dritte beobachtet werden, zum Beispiel einem Angehörigen, der den Patienten beim Arztbesuch begleitet oder einem geschulten Beobachter, der mit der Situation und mit der Methode vertraut ist. Zur Auswertung werden die Incidents in definierte Kategorien gefasst, um sie dann nach unterschiedlich komplexen Schemata zu bewerten. Typisch wäre etwa eine Analyse der Häufigkeit ihres Vorkommens innerhalb der Stichprobe (EMMUS 1999, FLANAGAN 1954). So sollen Defizite und / oder Stärken des Systems deutlich werden, um dort mit konkreten Veränderungen ansetzen zu können. Die Stärken der CIT liegen in ihrem direkten Bezug zu realen Situationen und in ihrer Spezifität: sie erfasst wichtige erlebte Elemente in definierten Situationen, nicht vage Vermutungen (BRADLEY 1992). Der Nachteil hingegen besteht darin, dass diese Methode abhängig ist von dem menschlichen Erinnerungsvermögen, $d$. $h$. die Wiedergabe kann schnell verzerrt und / oder unvollständig sein. Außerdem werden in der Regel nur herausragende Wahrnehmungen berichtet, seltene und negative Ereignisse sind daher tendenziell überrepräsentiert.

In dieser qualitativen Studie zur Beschreibung der Determinanten einer tragfähigen therapeutischen Beziehung wurden die Patienten durch die CIT angeregt, sich an ganz bestimmte Situation zu erinnern, in denen sich ärztliches und eigenes Verhalten auf die Beziehung zueinander ausgewirkt hat. Was hat zum Beispiel verärgert oder erfreut? Was waren die entscheidenden Verhaltensweisen des Arztes, die ihn als vertrauenswürdig erscheinen ließen, seine (fachliche) Autorität stützten oder entkräfteten? Entsprechende Ansätze wurden bei der Konzipierung des Leitfadens und der Durchführung der Interviews berücksichtigt.

\subsection{Durchführung der Untersuchung}

\subsubsection{Durchführung der Interviews}

Nach Erhalt der unterzeichneten Informed Consent-Bögen wurde telefonischer Kontakt zu den Patienten aufgenommen, um sie noch einmal kurz persönlich über den Ablauf des Interviews zu informieren und einen Termin und Treffpunkt zu vereinbaren. Die Wahl bezüglich des Orts des Treffens wurde dabei den Patienten überlassen. Dies sollte helfen, einen höchstmöglichen Grad an Wohlbefinden und Natürlichkeit für die Patienten zu erhalten (LAMNEK 1995, S. 57 f.). Die Interviews, die eine umfangreiche Kenntnis der Thematik, Fragestellung und Zielen des Projekts voraussetzten (HOPF 2000, S. 358), haben die Autorin dieser Arbeit und eine Mitarbeiterin des Projekts (Dipl.-Psych. A. Rogausch) durchgeführt.

Vor Beginn des eigentlichen Interviews wurden in einem kurzen Fragebogen Anhang A.2 personenbezogene und krankheitsspezifische Daten erhoben. Das Interview selbst wurde in seiner vollen Länge auf einer Audiokassette aufgezeichnet.

Der Verlauf des Interviews war an den Leitfaden angelehnt, jedoch ergaben sich Reihenfolge und Gewichtung der Themen aus dem natürlichen Gesprächsverlauf. So hatte der Pati- 
ent die Möglichkeit, inm relevant erscheinende Aspekte frei zu artikulieren und zu gewichten. Dies betrifft Aspekte, die zwar nicht antizipiert waren, aber dennoch interessant und wichtig für die Forschungsfrage sein können (MERTON und KENDALL 1993, S. 196; HOPF 2000, S. 359). Der Interviewer passte sich also dem Interviewten an, der das Gespräch inhaltlich und sprachlich gestaltete (LAMNEK 1995, S. 96). Nur bei Bedarf wurde gezielt nachgefragt und nach Erreichen des höchsten Antwortniveaus (Kompetenz) des Gesprächpartners das Thema gewechselt (AUFENANGER 1991, S. 43).

Nach Beendigung des Interviews hatten die Patienten Gelegenheit, Rückfragen zu stellen. Abschließend wurden die Formalitäten für die zu zahlende Aufwandsentschädigung erledigt.

\subsubsection{Datenerfassung}

Die Interviews wurden vollständig und im Originalwortlaut transkribiert. Die Transkription beinhaltete auch Notationszeichen, die Pausen, unverständliche Sprechpassagen und heraushebende Betonung einzelner Abschnitte erfassten (vgl. KOWAL und O'CONNELL 2000, S. 439), Namen von Personen und Orten wurden anonymisiert. Die Transkripte wurden doppelt, von verschiedenen Personen Korrektur gehört und gelesen, um eine möglichst hohe Übereinstimmung mit der Tonbandaufnahme zu erreichen. Nach der Korrektur wurden die entsprechend formatierten Transkripte in das Software Programm ATLAS.ti (MUHR 1997) zur Verwaltung und qualitativen Datenanalyse entwickelt eingelesen.

Eine Kopie des Transkripts wurden dem jeweiligen Patienten auf Wunsch zugesandt. Er hatte so auch die Möglichkeit zur Stellungnahme.

\subsection{Datenanalyse}

Datenerhebung und Datenanalyse sind in der qualitativen Forschung eng miteinander verknüpft. So haben zum einen die Auswertungsschritte im Rahmen der zirkulären Strategie direkten Einfluss auf die Datenerhebung, zum anderen ist die Auswahl der Auswertungsstrategien eng an das Datenmaterial, die Fragestellung und die methodische Anlage der Untersuchung gebunden (SCHMIDT C 2000, S. 447). Daraus ergibt sich, dass es nicht "die“ Methode zur Auswertung des Datenmaterials gibt, vielmehr muss eine gewisse Flexibilität erhalten bleiben, die Methode den Daten anzupassen, nicht die Daten der Methode. ROGERS et al. (1997, S. 41) fassen dies wie folgt zusammen:

"In the context of attention to ,meaning' qualitative methods need to be able to be responsive to circumstances as they exist, rather than attempt to create a situation in which the variable of interest can be controlled and their relationships examined. For this reason the hallmark of good qualitative methodology is its flexibility rather than its standardisation." 
Dennoch ist es erforderlich, ein gewisses $M a ß$ an regelgeleitetem Vorgehen einzuhalten und die einzelnen Schritte explizit darzulegen, um das Gütekriterium der Nachvollziehbarkeit qualitativer Forschung zu erfüllen (STEINKE 2000, S. 324 f.). Es wurde also in Anlehnung an existierende Theorien eine Auswertungsstrategie für diese Untersuchung entwickelt, die sich eng an einem Vorgehen von SCHMIDT C (2000, S. $447 \mathrm{ff}$.) orientiert und sich in folgende Schritte gliedern lässt:

- Materialorientiertes Erfassen von Themen

- Erstellung eines Kodierleitfadens (Codebook)

- Kodierung der Interviews

- Erfassen und Darstellen von Inhalten und Beziehungen

- Zusammenfassung der sich ergebenden Erkenntnisse.

Die Analyse erfolgte im ständigem Dialog innerhalb eines multiprofessionellen Teams (Medizinerin, Anthropologin, Psychologin). So konnten unterschiedliche, an die Persönlichkeit der Forscher gebundene Interpretationen abgeglichen, ergänzt und durch argumentative Auseinandersetzung definiert und gestützt werden.

Wesentlicher Bestandteil des analytischen Prozesses waren außerdem so genannte „analytische Memos“. Während des gesamten Vorgehens wurden Aspekte, die ins Auge fielen, Fragen aufwarfen, Interesse weckten und / oder einen möglichen Interpretationsansatz boten, formlos festgehalten, um später darauf zurückgreifen zu können.

\subsubsection{Materialorientiertes Erfassen von Themen}

In einem ersten Schritt der Auswertung wurden einige transkribierte Interviews wiederholt gelesen. Textpassagen, die für die Forschungsfrage relevant erschienen, wurden markiert und die sich aus dem Text ergebenden Themen wurden festgehalten (vgl. TYLOR und BOGDAN 1998, S. 143). Lenkend war hierbei das theoretische Vorverständnis zur Fragestellung. So entstand ein erster Eindruck davon, welche Aspekte aus Sicht der Patienten bedeutungsvoll waren. Für den weiteren Prozess stellten diese zunächst noch recht vage formulierten Kategorien die Grundlage zur Überarbeitung des Interviewleitfadens und erste Anhaltspunkte zur Erstellung eines Codebooks dar. SCHMIDT C (2000, S. 448) bezeichnet diese erste Phase als „materialorientierte Bildung von Auswertungskategorien“. Diese Gruppierung von Textbausteinen oder Zitaten ist als reduktiver Schritt zu verstehen, in dem eine erste thematische Gliederung vorgenommen und das Datenmaterial dadurch leichter handhabbar gemacht wird.

Daraufhin wurden die Transkripte in das Softwareprogramm ATLAS.ti (MUHR 1997) eingelesen. Hierbei handelt es sich um ein Programm zur Organisation qualitativer Daten im 
Interpretationsprozess. Seit nunmehr einigen Jahren wird der Einsatz von Computerprogrammen zur Unterstützung qualitativer Forschung empfohlen, um das Suchen und Navigieren in großen Datenmengen zu vereinfachen und insgesamt den Auswertungsprozess zu professionalisieren (FIELDING und LEE 1991, HUBER 1992).

\subsubsection{Erstellung eines Kodierleitfadens (Codebook)}

Grundlage für die Erstellung des Kodierleitfadens war die vorangegangene thematische Durchsicht der Interviews. Gemäß dieser Themen wurden weitere Transkripte durchgesehen und mit dem Computerprogramm markiert. Hierbei wurden selbstverständlich auch neu auftretende Themen aufgenommen. Die Themenliste wurde erneut gegliedert und in einem Kodierleitfaden zusammengefasst, welcher dann an einigen Interviews getestet und dem Bedarf entsprechend modifiziert wurde (CRABTREE und MILLER 1992, S. 95). Auch hier findet sich die zirkuläre Strategie wieder, da im Verlauf der Kodierung immer wieder materialorientiert Modifikationen vorgenommen wurden. Um eine möglichst große Reliabilität zu erreichen, wurde sowohl das Codebook als auch seine Anpassungen wiederholt in der multidisziplinären Gruppe diskutiert (HARDING und GANTLEY 1998). Die endgültige Form des Kodierleitfadens und die Definitionen der Codes finden sich in Anhang A.5 und A.6.

Die Erarbeitung des Codebooks und die genaue Definition der Codes ist als induktiver Schritt zu verstehen. Hierdurch lassen sich Textpassagen eindeutig zuordnen und die Strukturierungsarbeit präzisieren (MAYRING 1995, S. 213). Der Kodierleitfaden stellt außerdem die Grundlage für eine weitere Bearbeitung des Materials, der Strukturierung in Form des Kodierens, dar.

\subsubsection{Kodierung der Interviews}

Beim Kodieren wurden die vorher aus dem Material gewonnenen Auswertungskategorien wieder auf das Material angewandt. Anhand des Kodierleitfadens wurden alle Transkripte wiederholt durchgesehen und die relevanten Textpassagen markiert und den Kategorien zugeordnet (SCHMIDT C 2000, S. 452). Dies geschah einerseits auf Textebene in den vollständigen Interviews, andererseits auf Zitatebene, indem die kodierten Zitate durchgesehen und ihre Zuordnungen überprüft und angepasst wurden. Im Rahmen dieser Zuordnung bzw. der sich ergebenden Unsicherheiten mit einzelnen Passagen wurde auch hier wieder der Kodierleitfaden überarbeitet, auf die Forschungsfrage zugespitzt und mit Hilfe von Diagrammen (Anhang A.7) die Verhältnisse der Kategorien zueinander und zum Forschungsziel dargestellt (TYLOR und BOGDAN 1998, S. 148 f.). Abschließend wurden noch einmal alle Interviews gelesen und anhand der letzten Fassung des Kodierleitfadens verschlüsselt.

Während der Kodierung wird bewusst ein Informationsverlust hingenommen, der jedoch umso geringer bleibt, je klarer und differenzierter die Kategorien definiert werden (SCHMIDT) C 2000, S. 453). 


\subsubsection{Erfassen von Inhalten und Beziehungen}

Als nächster Schritt wurden die den Codes zugeordneten Zitate noch einmal losgelöst aus ihrem Kontext betrachtet und inhaltlich entsprechend ihrer Aussagen und Dimensionen gruppiert. Mit dieser Tätigkeit entstanden im Rahmen eines Codes eine Reihe von Subcodes, denen die Zitate zugeordnet werden konnten. Anschließend wurden die Zitate der Subcodes noch einmal betrachtet und ihre Zuordnung überprüft. Dabei war nicht entscheidend, dass eine Aussage von möglichst vielen Zitaten gestützt werden sollte, vielmehr ging es darum die gesamte Bandbreite der Aussagen zu erfassen. Tabelle 4.1 zeigt dies exemplarisch auf.

Tabelle 4.1: Code „Noncompliance Information“

\begin{tabular}{|l|l|}
\hline Code & „Noncompliance und Information“ \\
\hline Definition & $\begin{array}{l}\text { Erfahrungen mit Compliance / Noncompliance und deren Folgen, die Einfluss } \\
\text { auf die weitere Compliance haben. Eigene Erlebnisse und Erfahrungen im } \\
\text { Zusammenhang mit dem speziellen Medikament, der Medikamentenklasse } \\
\text { oder Medikamenten im Allgemeinen. }\end{array}$ \\
\hline Inhalt & $\begin{array}{l}\text { - Der Patient sollte über die Wirkungsweise des Medikaments informiert sein. } \\
\text { - Der Patient sollte über die unerwünschten Wirkungen informiert sein. (Sie } \\
\text { verlieren daher einen Teil ihrer Bedrohlichkeit.) } \\
\text { - Die Informationen, die der Beipackzettel enthält, sind in Umfang und Ver- } \\
\text { ständlichkeit ausreichend. } \\
\text { - Voneinander abweichende Informationen aus unterschiedlichen Quellen } \\
\text { verunsichern. } \\
\text { - Der Arzt sollte therapeutische Alternativen aufzeigen. } \\
\text { - Die Gründe für die Verordnung der Medikamente sollten vom Arzt transpa- } \\
\text { rent gemacht werden. }\end{array}$ \\
& \\
\hline
\end{tabular}

Die feinere Untergliederung der Codes hatte eine deutlich verbesserte Handhabung des Datenmaterials zur Folge und machte so den nächsten Auswertungsschritt möglich: Um die erfassten Inhalte mit einer Bedeutung belegen zu können, wurden sie zu den zentralen Fragestellungen und zueinander in Beziehung gesetzt. Dies geschah in Anlehnung an TYLOR und BOGDAN (1998, S. 148) in Form von Diagrammen unterschiedlicher Art. In diesem Schritt der Analyse lenken die oben erwähnten Memos (Abschnitt 4.6 die Aufmerksamkeit beim Betrachten von Zusammenhängen; zeitgleich wird das Datenmaterial vor dem Hintergrund der Memos noch einmal genauer betrachtet. Unterschiedliche Beispiele wurden gegenübergestellt und nach stützenden bzw. kontrastierenden Argumenten gesucht.

Abschließend werden die sich aus den vorangegangenen Schritten ergebenden Erkenntnisse als Ergebnisse zusammengefasst. In diesem Schritt kommt es zur Generierung vorläufiger Hypothesen. 


\subsection{Datenschutz und Patientenaufklärung}

Die Studie, die neben der hier durchgeführten Befragung der Patienten auch eine Befragung Angehöriger umfasste, wurde durch die Ethik-Kommission der Medizinischen Fakultät der Georg-August-Universität Göttingen genehmigt (Antragsnummer 15/3/02). Die Einhaltung des Datenschutzes war durch Entkopplung der Patienten von den vermittelnden Praxen und Einrichtungen sowie durch die anonymisierte Dokumentation und Archivierung der Interviews gewährleistet. Die Patienten erhielten im Vorfeld ein Informationsblatt mit Einverständniserklärung (Informed Consent, Anhang A.1), in dem das Forschungsprojekt mit seinen zentralen Fragestellungen vorgestellt wurde. Ihr Einverständnis beruhte auf Freiwilligkeit und konnte jederzeit widerrufen werden. 


\section{Ergebnisse}

\subsection{Beschreibung der Stichprobe}

In die Untersuchung wurden 20 Patienten (9 Frauen, 11 Männer) mit der Diagnose Schizophrenie aufgenommen. Tabelle 5.1 listet die interviewten Personen auf. ${ }^{1}$

Tabelle 5.1: Patientenstichprobe

\begin{tabular}{|c|c|c|c|c|c|c|c|c|}
\hline Atlas & $\begin{array}{c}\text { Patient } \\
\text { Nr. }\end{array}$ & $\begin{array}{r}\text { Datum } \\
\text { Interview }\end{array}$ & $\begin{array}{c}\text { Alter } \\
\text { [Jahre] }\end{array}$ & Geschlecht & $\begin{array}{c}\begin{array}{c}\text { Krankheits- } \\
\text { dauer }\end{array} \\
\text { [Jahre] }\end{array}$ & $\begin{array}{c}\text { Hausarzt } \\
\text { bekannt } \\
\text { seit } \\
\text { [Jahren] }\end{array}$ & $\begin{array}{c}\text { Facharzt } \\
\text { bekannt } \\
\text { seit } \\
\text { [Jahren] }\end{array}$ & $\begin{array}{l}\text { Diagnose } \\
\text { (eigene Worte) }\end{array}$ \\
\hline 1 & 105 & 16.03 .02 & 52 & $\mathrm{~m}$ & 35 & 1,5 & 10 & Psychose \\
\hline 2 & 113 & 06.06 .02 & 38 & $\mathrm{~m}$ & 12 & 8 & 2 & manische Psychose \\
\hline 3 & 114 & 06.12 .01 & 73 & w & 43 & 20 & - & Nervenkrankheit \\
\hline 4 & 109 & 08.03 .02 & 30 & $\mathrm{~m}$ & 04 & 25 & 0,5 & paranoide Schizophrenie \\
\hline 5 & 106 & 16.03 .02 & 55 & w & 26 & 39 & 10 & Psychose \\
\hline 6 & 107 & 08.03 .02 & 36 & $\mathrm{~m}$ & 25 & 1,5 & 3 & $\begin{array}{l}\text { latente Schizophrenie mit } \\
\text { psychotischen Episoden }\end{array}$ \\
\hline 8 & 115 & 09.07.02 & 38 & w & 11 & 6 & 0,2 & $\begin{array}{l}\text { Psychose aus schizo- } \\
\text { phrenem Formenkreis }\end{array}$ \\
\hline 9 & 111 & 11.05 .02 & 26 & $\mathrm{~m}$ & 12 & 7 & 7 & Schizophrenie \\
\hline 10 & 110 & 09.03.02 & 62 & $\mathrm{~m}$ & 44 & 20 & 3 & Schizophrenie \\
\hline 11 & 103 & 03.03.02 & 23 & w & 01 & 5 & 0,4 & Psychose \\
\hline 14 & 116 & 10.07.02 & 36 & $\mathrm{~m}$ & 16 & 6 & 6 & $\begin{array}{l}\text { paranoide halluzinatori- } \\
\text { sche Psychose aus schi- } \\
\text { zophrenem Formenkreis }\end{array}$ \\
\hline 15 & 102 & 20.02 .02 & 79 & $\mathrm{~m}$ & 22 & 40 & - & Nervenkrankheit \\
\hline 16 & 101 & 04.02 .02 & 50 & w & 05 & 5 & 5 & Psychose \\
\hline 17 & 112 & 27.05 .02 & 49 & $\mathrm{~m}$ & 26 & 5 & 1 & Schizophrenie \\
\hline 18 & 117 & 11.09 .02 & 54 & w & 16 & 4 & 6 & $\begin{array}{l}\text { Psychose aus dem Be- } \\
\text { reich der paranoiden } \\
\text { Schizophrenie }\end{array}$ \\
\hline 19 & 104 & 16.03 .02 & 35 & w & 15 & 2 & 4 & Psychose \\
\hline 20 & 100 & 04.02 .02 & 61 & w & 45 & 18 & 20 & $\begin{array}{l}\text { schizophren mit Angstzu- } \\
\text { ständen }\end{array}$ \\
\hline 21 & 108 & 08.03 .02 & 22 & w & 03 & 5 & 0,5 & schizophrene Psychose \\
\hline 22 & 118 & 14.10 .02 & 44 & $\mathrm{~m}$ & 24 & - & 10 & Psychose \\
\hline 23 & 119 & 22.10 .02 & 41 & $\mathrm{~m}$ & 22 & 6 & 4 & Psychose \\
\hline
\end{tabular}

\footnotetext{
${ }^{1}$ In ATLAS.ti wurden die Nummern 7, 12 und 13 nicht vergeben, da die ursprünglich unter diesen Nummern eingelesenen Transkripte nicht dem erforderlichen Format zur weiteren Handhabung entsprachen. Die Transkripte wurden nach der Anpassung des Formats unter anderen Nummern erneut in das Programm eingelesen. Die „Patientenummern“ ermöglichen eine Zuordnung der Interviews zu der anonymisierten Archivierung als Audiokassette und Transkript außerhalb von ATLAS.ti.
} 
Das Durchschnittsalter betrug 45,2 Jahre (Range [R]: 22-79 J.; Standardabweichung [SD]: 15,78), die mittlere Krankheitsdauer 20,4 Jahre (R: 1-45 J.; SD: 13,52). Im Mittel hatten diese Patienten 7,5 stationäre Aufenthalte (R: 2-19; SD: 4,94) hinter sich gebracht. Die meisten Patienten wurden sowohl vom Hausarzt als auch vom Facharzt betreut. Lediglich drei Patienten konsultierten nur einen Arzt, in einem Fall war dies der Psychiater, in zweien der Hausarzt. Jedoch wurden alle Patienten auch zwischenzeitlich stationär behandelt, was die angegebene Versorgungssituation relativiert. Der Hausarzt war im Durchschnitt seit 11,8 Jahren bekannt (R: 1,5 -40 J.; SD: 11,96), der Psychiater seit 5,1 Jahren (R: 0,2-20; SD: 5,2). Die Hälfte der Patienten kannte ihren Hausarzt schon vor Beginn der psychischen Erkrankung, während der Kontakt zum Psychiater erst mit der Erkrankung hergestellt wurde. Im Falle einer Doppelversorgung war die Kontakthäufigkeit mit dem Hausarzt geringer. Als Ansprechpartner für psychische Probleme wurde stets der Psychiater angegeben.

Medikamentös waren neun Patienten mit einem atypischen Neuroleptikum eingestellt, drei erhielten ein typisches Neuroleptikum und fünf ein nicht weiter bezeichnetes Depotpräparat. Drei Patienten erhielten zum Zeitpunkt der Erhebung keinerlei antipsychotische Medikation.

Tabelle 5.1 ist auch Grundlage der im Folgenden zitierten Interviewausschnitte. Zum Beispiel setzt sich die Bezeichnung Q 1:2 wie folgt zusammen: Q steht für Quotation (engl. Zitat), 1 für die Nummer des Interviews in ATLAS.ti und 2 für die Zitatnummer innerhalb des Interviews. Zudem sind jeweils Alter und Geschlecht angegeben. Innerhalb der Zitate bezeichnet „P“ die Aussagen des Patienten, „।“ Fragen bzw. Kommentare des Interviewers. Außerdem wurden die folgenden Notationszeichen verwendet:

- - - = Sprechpause

- _ = Satzabbruch durch den Sprecher

- $(\ldots)=$ unverständliche Passage auf dem Tonband

- $[\ldots]=$ durch die Autorin vorgenommene Auslassungen

- $[$ Wort $]=$ durch die Autorin eingefügte Wörter.

\subsection{Zuständigkeit des Hausarztes}

Wie im Vorfeld beschrieben, wurden auch die interviewten Patienten in den meisten Fällen sowohl vom Facharzt als auch vom Hausarzt betreut. In der Regel hatten sie recht genaue Vorstellungen davon, welche Aufgaben der jeweilige Arzt übernehmen und welche Kompetenzen er haben sollte. Patienten, die nur ein Arzt betreute, vereinten diese Zuständigkeitsbereiche mit unterschiedlicher Gewichtung auf eine Person. 


\subsubsection{Der Hausarzt als Gatekeeper}

Für viele der Patienten war der Hausarzt im Zentrum ihrer Gesundheitsversorgung als Gatekeeper angesiedelt. Er war für sie erster Anlaufpunkt bei gesundheitlichen Problemen, leitete sie in die eventuell notwendige fachärztliche Betreuung weiter und erhielt dann Rückmeldungen von den Fachkollegen. Die Patienten wünschten sich daher von ihm auch ausreichende Kenntnis der Psychopathologie, damit er psychische Störungen erkennen und adäquat darauf reagieren könnte.

[P:] Und ich sage deswegen schlechte Erfahrungen, weil ich die, äh, Feststellung machen konnte, äh, dass diese beiden Hausärzte bei denen, die mich damals behandelt haben, sich überhaupt offenbar nicht genügend in Psychopathologie ausgekannt haben. Beispielsweise geht das allein schon daraus hervor, [...] dass die mich beide wegen psychischer Beschwerden zum Neurologen schicken wollten. Statt zum Psychologen, Psychiater und Psychotherapeuten. (Q 1:76; 52 J., m)

[P:] ...dass der Hausarzt mehr Verantwortung auch trägt, für die einzelnen Patienten jetzt, als Ansprechpartner, als Vermittler zu Fachärzten und so -- das finde ich schon wichtig.

I: Könnten Sie sich das vorstellen?

$P$ : Ja, ja. Ich glaube, das ist schon notwendig. Außerdem können die vorab schon viel mehr abchecken vielleicht - - äh --, was vielleicht für Ursachen vorliegen auch. Grad bei psychischen Erkrankungen, ne. - - Dass man sagen kann, der hat vielleicht das jetzt, oder Depressionen, dass man das besser erkennt. Das wissen manche Ärzte vielleicht auch nicht so genau, wie man das erkennen kann. (Q 6:93; $36 \mathrm{~J} ., \mathrm{m})$

[P:] Weil ich denke es landen halt viele - - also gerade bei so Ersterkrankungen, da landet man auch häufig dann beim Hausarzt. - - [...] Ansonsten ist es halt ne nette Sache, wenn die Hausärzte dann nicht irgendwie anfangen herumzubasteln, und es funktioniert halt nicht. Also ich weiß auch nicht, ob Hausärzte jetzt unbedingt zum Beispiel Psychopharmaka verschreiben sollten, weil sie da auch häufig nicht so viel Ahnung von haben. (Q 9:94; $26 \mathrm{~J} ., \mathrm{m})$

Aus dieser Schlüsselposition ergab sich auch die wichtige Aufgabe der Kommunikation mit dem Patienten einerseits und mit weiteren an der Therapie beteiligten Fachpersonen andererseits. Der Hausarzt sollte über die anderweitigen Behandlungen seines Patienten informiert sein und die inm zur Verfügung stehenden Informationen im professionellen Umfeld kommunizieren. Im Idealfall hatte der Hausarzt ein Gesamtbild der individuellen Gesundheitssituation. Außerdem sollte er - soweit erforderlich - dem Patienten fachliche Informationen verständlich mitteilen.

P: Ja, ja. Der Hausarzt kennt meine ganze Geschichte. Und auch meine Psychiaterin schickt irgendwas zu meinem Hausarzt. Also Unterlagen. Ja. Dass ein Arzt alles zusammen hat.[...] Das gefällt mir gut, ja. Ich brauch nur zu meinem Hausarzt gehen und der hat die ganzen Unterlagen. (Q 5:43; $55 \mathrm{~J} ., \mathrm{w})$ 
P: Also was ich vom Hausarzt verlangen kann, ist vielleicht, dass er sich ein bisschen um die Kommunikation zwischen den behandelnden Ärzten kümmert, und dass er vielleicht auch guckt, dass wenn der Kranke nicht in der Lage ist, sich selbst zu organisieren, dass er einen guten Betreuer kriegt. Das kann der Arzt. Das ist vielleicht noch vom Arzt zu erwarten, dass er da guckt, dass der Mensch nicht allein auf sich alleine gestellt ist. (Q 19:14; 35 J., w)

[P:] Es ist ja teilweise so, dass viele Ärzte, Hausärzte, gar keine Ahnung haben, wo die Patienten hingehen, und das finde ich nicht so gut. Weil letztlich kann die optimale Behandlung ja gar nicht stattfinden. (Q 19:16; 35 J., w)

\subsubsection{Der Hausarzt als Körperarzt}

Für eine große Anzahl der Patienten nahm der Hausarzt primär die Rolle eines Körperarztes ein. Patienten, die in regelmäßiger psychiatrischer oder psychotherapeutischer Behandlung waren, formulierten dies oft auch als alleinigen Anspruch an den Hausarzt. Die Mehrzahl der Patienten ging mit einem konkreten somatischen Problem zum Hausarzt und sah seine Aufgabe in dessen Behandlung. Auch wenn es in der Situation durchaus zu einem persönlicheren Gespräch kommen konnte, so war das Bedürfnis danach nicht der primäre Anlass des Besuchs.

P: Also wenn ich zum Hausarzt gehe, habe ich konkret in der Regel ein körperliches Problem. Das heißt, ich hab Durchfall oder Magenschmerzen, eine Erkältung. Ich habe auch nie so konkret größere Sachen. Ich muss halt regelmäßig gehen, weil ich halt Probleme mit der Schilddrüse hab, und eigentlich sind das meine regelmäßigen Arztbesuche. Und wenn ich dann zu dieser Zeit irgend ein Problem hab, dann spreche ich es an. (Q 19:12; $35 \mathrm{~J} ., \mathrm{w})$

In einigen Fällen empfanden es die Patienten so, dass sich der Hausarzt vollständig aus der psychischen Betreuung zurückzog. Dies wurde zum Teil bedauert, zum Teil aber auch so gewünscht.

[P:] Könnte sich das eine oder andere Mal ruhig etwas mehr Zeit nehmen und - - noch zu einem kleinen Gespräch, aber ansonsten will ich das vielleicht auch meistens gar nicht. Dass er mir eben hilft, die Beschwerden, die grade existieren, halt zu lindern, und damit ist es auch dann gut so, würde ich mal sagen. (Q 23:10; $41 \mathrm{~J} ., \mathrm{m})$

[P:] - - ich hab schon manchmal das Bedürfnis, mit ihm bisschen mehr noch zu reden. Ich trau mich dann aber auch nicht so - - ich will dem Arzt dann auch nicht so dauernd was erzählen, und - - na ja, es ist - -

I: Aus welchem Grund genau?

$P$ : Ich weiß manchmal auch nicht, wie ich das sagen soll. Ich hab manchmal auch so das Gefühl, manche Ärzte die wollen auch gar nicht so viel mit mir darüber reden, die denken, die haben die Diagnose, und der Arzt, der Psychiater, wird sich schon drum kümmern, der hat ja genug Zeit zum Reden. (Q 6:90; 36 J., m) 


\subsubsection{Weiterführen der Pharmakotherapie durch den Hausarzt}

Im Rahmen der Schizophreniebehandlung war der Hausarzt oftmals in die Pharmakotherapie eingebunden. Bei einer Patientin führte er sie selbständig durch, passte Dosierungen an oder stellte auf ein anderes Medikament um, bis Schwierigkeiten auftraten, die das Hinzuziehen eines Fachkollegen notwendig machten.

[P:] Ich war erst ein bisschen geknickt, als er meinte, ich sollte jetzt noch einen Facharzt aufsuchen, [...]. Das hatte er mir aber auch schon ganz am Anfang unserer Betreuung gesagt, als ich gesagt, äh, gefragt habe: Können Sie mich denn mit dem Krankheitsbild auch behandeln? - da hatte er gleich gesagt: Ja, aber wenn es irgendwie, wenn jetzt irgendwelche Schwierigkeiten auftreten oder so, dann müssten wir noch einen Facharzt hinzuziehen. Und so ist das jetzt praktisch auch gekommen. (Q 8:68; $38 \mathrm{~J} ., \mathrm{w})$

In den meisten Fällen führte jedoch der Hausarzt die vom Psychiater verordnete Therapie weiter. Seine Aufgaben waren dann das Ausstellen von Folgerezepten sowie die Überwachung und ggf. Eindämmung von unerwünschten Wirkungen. Er kontrollierte somatische Parameter wie Blutbild, Blutdruck, Plasmaspiegel des Medikaments etc. Dies war insbesondere bei der Weiterbehandlung im Anschluss an einen stationären Aufenthalt und bei Patienten, die ein psychologischer Psychotherapeut oder anderes Fachpersonal ambulant betreuten, der Fall.

I: Also Sie sagen der Hausarzt hat mit der Sache eigentlich nichts zu tun oder nicht so viel zu tun.

P: Eigentlich nix. Also ich war einmal da wegen der Überweisung für die Institutsambulanz und einmal für das Rezept für meine Neuroleptika. (Q 11:32; $23 \mathrm{J.}$, w)

I: Und wie oft gehen sie ungefähr hin? [zum Hausarzt]

P: Wenn die Tabletten weg sind, gehe ich wieder hin und lass mir sie verschreiben. Und dann auch [...] Blut abnehmen muss. Weil ich die Tablette nehme, muss das Blut kontrolliert werden und zum Blutdruck messen, Zucker messen. (Q 3:34; $73 \mathrm{~J} .$, w)

Eine Patientin nahm ihre Hausärztin zudem noch in Anspruch, um eine zweite Meinung über die vom Facharzt verordnete Therapie einzuholen.

I: Ah, ja! Was besprechen Sie dann mit der Hausärztin?

$P$ : Ob sie meint, dass die Tabletten mir wirklich gut tun, und es ist aber nichts Gegenteiliges gesagt worden, also die Tabletten, meine Psychiaterin hat mir gesagt, dass die mir gut helfen, und meine Hausärztin auch. Und wenn ich zwei höre, gefällt mir das besser. (Q 5:55; 55 J., w)

\subsubsection{Der Hausarzt als Ansprechpartner für psychische Probleme}

Neben seinen Aufgaben für die somatische Gesundheit des Patienten spielte der Hausarzt aber auch für die psychische Gesundheit eine Rolle. Dies lag zum einen daran, dass 
Patienten einen Zusammenhang zwischen körperlichem und seelischem Wohlbefinden sahen, zum anderen, dass der Hausarzt Informationen über die psychische Behandlung erhielt und zum Teil auch darin involviert war (beispielsweise in die Pharmakotherapie; vgl. AbSchnitt 5.2.3). Insgesamt bestand also von Seiten der Patienten Bedarf, mit dem Hausarzt auch über persönliche und psychische Probleme sprechen zu können.

[P:] Ich bin immer nur, wenn ich kleine körperliche Beschwerden hatte, zu ihm gegangen. Ich würde mir aber wünschen --, ähm, mal mit dem Hausarzt ein längeres Gespräch über meine psychischen Probleme zu führen. Und, äh, ich hatte eigentlich immer einen Hausarzt gesucht, der sich für diese Dinge etwas Zeit nimmt. Da lege ich allergrößten Wert darauf. Ja. (Q 1:90; 52 J., m)

Eine besondere Rolle kam dem Hausarzt auch bei Erkrankungsbeginn zu. Während der Patient sich in der neuen Situation zurechtfinden musste, war der Hausarzt Informationsund Orientierungshilfe im Lernprozess zur Bewältigung der Erkrankung. Hier war neben der Funktion als Gatekeeper auch „menschlich-persönliche“ Kompetenz gefragt. Dies traf einerseits für diejenigen Hausärzte zu, die dem Patienten schon vor Erkrankungsbeginn bekannt waren, jedoch auch für diejenigen, die vom Patienten im Anschluss an einen stationären psychiatrischen Aufenthalt zur Weiterbehandlung aufgesucht wurden.

[P:] Sie hat halt weiterhin für mich ein, sag ich mal, ein angenehmes Klima gehabt, wo ich mich halt auch -- aussprechen konnte, und ich muss dann zu Anfang, weil diese Krankheit für mich auch was ganz Neues war und sehr schwer umwälzend, musste ich mich natürlich zuerst mal auf ihre Aussagen und Empfehlungen verlassen. (Q 4:63; $30 \mathrm{~J} ., \mathrm{m})$

[P:] Und ich habe gelernt immer mehr_ - - Frühzeichen zu erkennen und - - äh - und ich denke mir, da war in der gewissen Zeit, mein Hausarzt hatte da eine wichtige Funktion, denn er hat mit mir geguckt, ob das Frühzeichen gibt. Oder wenn ich mal nicht wusste, ist das jetzt das schon ein bisschen spinderich oder ist das schon gefährlich, dann habe ich ihm, habe ich ihn aufgesucht und der hat mir das dann eben_ _- er musste mich dann auch sehen. (Q 8:73; $38 \mathrm{~J} ., \mathrm{w})$

\subsection{Zuständigkeit des Facharztes}

Ähnlich wie beim Hausarzt, sahen die Patienten auch bei ihrem Facharzt Zuständigkeiten in verschiedenen Bereichen. Die Mehrzahl der in den Interviews benannten Aufgaben entstanden aus seiner fachlichen Spezialisierung, aber auch für alltäglichen Fragen und Probleme war er für die Patienten von Bedeutung.

\subsubsection{Der Facharzt als Spezialist für die psychische Erkrankung}

Der Facharzt ist Spezialist für psychische Erkrankungen und folglich sahen die Patienten seine primäre Aufgabe in der Betreuung ihrer Schizophrenie. Dabei ließ sich die Betreuung 
grob in einen schulmedizinischen und einen (psycho-)therapeutischen Anteil aufteilen.

Im Rahmen der psychotherapeutischen Betreuung war der Facharzt zum einen Ansprechpartner für psychische Probleme, zum anderen fanden therapeutische Gespräche allein oder in der Gruppe statt. Die Behandlung beim Psychiater war primär auf die psychische Problematik ausgelegt, d. h., er hatte in der Regel mehr Zeit für Gespräche bzw. der Kontakt bestand aus geplanten Gesprächen. Diese Ausrichtung und das Verständnis vom Facharzt als Spezialist für die Psyche war auch Voraussetzung dafür, dass eine Atmosphäre entstehen konnte, in welcher der Patient sich auch auf einer sehr privaten Ebene öffnen konnte. In diesem Teil der Behandlung gab es häufige Überschneidungen mit den Aufgaben von anderen an der Therapie beteiligten Personen, wie etwa psychologischen Psychotherapeuten oder Pflegepersonal mit Zusatzausbildung, zum Teil wurde diese Behandlung auch ganz von ihnen übernommen.

[P:] Und dann Probleme eigentlich so im Alltag oder außerhalb der Erkrankungsschübe sind eigentlich auch nicht da, mein Leben geht halt mehr so ein bisschen gleichförmig irgendwie, und was mir während der Woche halt auffällt, Psychisches so an Konflikten oder so, das hebe ich mir halt dann auf für die Sitzung mit dem psychologischen Psychotherapeuten. (Q 4:58; $30 \mathrm{~J} ., \mathrm{m})$

[P:] [...] Beim Psychiater geht es in die Vollen, gleich in die Gespräche und so um die innersten Gefühle und -- das kostet ja, da braucht man ja auch ein bisschen Zeit dafür, um sich zu öffnen. Selbst in einer Stunde, die man dann Zeit hat. Dass man auch nicht gleich in den ersten Minuten gleich loslegen [kann] und das ist dann beim Hausarzt grade die Grenze, wo dann da vielleicht der nächste Patient schon wieder kommt, und da kann man dann sich auch nicht so - - sich so auslassen. (Q 6: 24; $36 \mathrm{~J} ., \mathrm{m}$ )

I: Und wenn Sie zum Psychiater gehen, ist es anders? Oder?

P: Nein. Schon. Der hat halt seine Fachausbildung und-- und er hat auch Zeit zum Zuhören, sozusagen. Und kennt mich auch entsprechend besser, aufgrund der vielen Sitzungen, die wir schon zusammen_ und Gespräche halt, ne, die wir zusammen geführt haben. (Q 23:11; 41 J., m)

Andererseits war der Facharzt für die medikamentöse Seite der Psychose-Behandlung zuständig. Er klärte auf, führte die Pharmakotherapie durch und betreute den Patienten, wenn unerwünschte Wirkungen auftraten. Seine Aufgabe sahen einige der Befragten auch darin, den Verlauf der Erkrankung zu begleiten und positiv zu beeinflussen. So könnten etwa die Erkennung von Frühzeichen eines drohenden Schubes und entsprechende Interventionen helfen, eine Hospitalisation zu verhindern. Außerdem konnte auch der Psychiater Ansprechpartner für körperliche Probleme sein. Dies gaben vor allem die Patienten an, die einen regelmäßigen Kontakt mit dem Psychiater, jedoch einen eher spärlichen mit dem Hausarzt hatten. Hier kam es also zu Überschneidungen mit den Aufgaben des Hausarztes, die bei einem Patienten sogar soweit gingen, dass der Psychiater als „Allgemeinarzt“, d. h. als jemand, der etwas von Medizin versteht, bezeichnet wurde. 
[P:] Aufgabe? Aufklären vielleicht, inwieweit er sich mit Nebenwirkungen auskennt, weil mit Nebenwirkungen hatte ich auch schon ein paar unangenehme Erfahrungen gemacht. Bei meinem jetzt, sagte er mir, müsste alle zwei Monate das Blutbild äh --kontrolliert werden. Da war ich erst ein bisschen beunruhigt, aber er hat mich gleich aufgeklärt, dass er--dieses Medikament viel einsetzt und--damit eigentlich_, davon noch nie was mitgekriegt hat. (Q 8:86; $38 \mathrm{~J} ., \mathrm{w})$

[P:] Aber er hat mir ja einfach aufgezeigt, was auch eine Aufgabe sein könnte, wenn man, wenn das nicht zuviel erwartet ist, also Frühsymptome äh - - zu erkennen, [...]-auch wenn es noch nicht - - - akut ist, so eine Phase, sondern davor, um eben äh - so was abzufangen und das - - da sieht sich ja jetzt grade die Institutsambulanz und andere Fachärzte vielleicht auch, um eben einen Krankenhausaufenthalt zu verhindern. (Q 8:84; $38 \mathrm{~J} ., \mathrm{w})$

[P:] wenn ich sowieso bei meinem Psychiater bin und eine medizinische Frage habe, den dann auch frage. Ist ja Allgemeinmediziner, also - - hat ja Medizin studiert und hat dann ja auch Ahnung von anderen Sachen, nicht nur von Psychiatrie. [...] (Q 9:92; 26 J., m)

In seiner Funktion als Fachmann auf dem speziellen Gebiet der psychiatrischen Erkrankungen arbeitete der Facharzt in der Regel nur in einem eingeschränkten Bereich der Gesundheitsversorgung des Patienten, an der auch andere Personen beteiligt waren. Die Patienten wünschten daher, dass er auch eine gute Kommunikation zu den anderen Behandelnden pflegte.

P: Oh, das weiß_kann ich Ihnen jetzt gar nicht sagen, ob da überhaupt Kontakt existiert.

I: Oder wie würden Sie sich das wünschen?

P: Zwischen Hausarzt und, Gott, sicher. Ich habe da in sofern keine Geheimnisse. Wenn es nach mir ginge, dürften die ruhig meine Daten austauschen. Diese beiden speziellen Personen. [...] (Q 23: 13; $41 \mathrm{~J} ., \mathrm{m})$

\subsubsection{Der Facharzt als Ansprechpartner für Probleme des Alltags}

Neben der fachärztlichen Betreuung war der Facharzt für einige Befragte auch jemand, der sie in den alläglichen und zum Teil auch persönlichen Belangen unterstützte. Er kannte die Lebenssituation des Patienten und war Ansprechpartner bei auftretenden Schwierigkeiten im Privatleben, in der Familie oder im Beruf. Ebenso unterstützte er auch in offiziellen Angelegenheiten, z. B. bei Krankschreibungen oder behördlichen Angelegenheiten.

[P:] Dann geht es zum Teil um die häusliche Situation, wie ich wohne und ob ich die Wohnung über einen langen Zeitraum behalten kann. Und dass ich die finanzielle Situation (...). Das brauche ich auch nicht, das kann ich am Arbeitsplatz mit meinem Vorgesetzten machen. Und die körperliche Situation, dass ich belastbar bin und die Belastung vertragen kann und einteile. Dass ich mich dabei wohl fühle und dergleichen mehr. (Q 22:12; $44 \mathrm{~J} ., \mathrm{m})$ 
[P:] Ansonsten gehe ich halt zum Psychiater, wenn irgendwas Amtliches zu erledigen ist, wegen Uni oder Schule oder sonst was da - - solche Sachen, das regele ich halt da.

- - Irgendwelche Krankschreibungen, das mache ich eigentlich nur über den Psychiater.

(Q 9:65; $26 \mathrm{~J} ., \mathrm{m})$

\subsection{Einflussfaktoren auf Compliance}

Aus den Interviews ergaben sich drei Hauptaspekte, die Einfluss auf die Medikamentencompliance der schizophrenen Patienten hatten: patientengebundene Faktoren, ärztliche Faktoren und äußere Einflüsse. In Abbildung 5.1 werden sie graphisch zueinander in Beziehung gesetzt.

\subsubsection{Persönliche Einstellung gegenüber Psychopharmaka}

Unter dem Begriff „Einstellungen gegenüber Psychopharmaka“ werden persönliche Meinungen und Vorbehalte gegenüber den Medikamenten zusammengefasst. Solche Einstellungen konnten sich einerseits aus den individuellen Erfahrungen ergeben, andererseits aus der Persönlichkeit und dem Weltbild des Patienten. Wiederholt nannten Patienten eine oft nicht genauer erklärbare Aversion gegen die Medikamente, sie wurden als unnatürliche und potentiell schädliche, chemische Substanzen eingeschätzt, und es bestand eine Furcht vor möglichen Gesundheitsschäden, die durch eine dauerhafte Einnahme entstehen könnten.

[P:] Die andere Seite der Medaille ist die, dass mir von fachärztlicher Seite gesagt wurde, auf meine Frage hin über die Schädlichkeit oder Unschädlichkeit von Psychopharmaka, dass, ja äh, dass Psychopharmaka auch eine gewisse Schädlichkeit haben. [...] da bin ich sehr kritisch, äh hab eine sehr skeptische Einstellung bei der Dauerverabreichung von Psychopharmaka. (Q 1:85; 52 J., m)

[P:] Ich meine Medikamente sind Chemie und so weit ist die Menschheit noch nicht, dass sie das optimale Medikament für jede Krankheit erfunden hat und da man nicht weiß, wie sich das auswirkt, finde ich, sollte man Medikamenten nur nehmen, wenn es auch sein muss. (Q 19:33; 35 J., w)

Insgesamt wurden die Medikamente jedoch als das kleinere Übel in Abgrenzung zum akuten psychotischen Schub gesehen. Sie ermöglichten eine gewisse Normalität in der Gestaltung des Alltags. Dies wurde als positiv erlebt und als erstrebenswert angesehen.

[P:] Also, ich bin dadurch, dass ich eben einsehen musste, dass ich eben doch ein bisschen schwer erkrankt bin, ein bisschen - - ja, kleinlauter, demütiger geworden, wenn es um Medikamente geht. Also, früher war ich da wirklich kritischer. Bloß was soll man machen? Bei dieser Krankheit muss man mit Chemie ran, das andere geht nicht und -(Q 8:104; $38 \mathrm{~J} ., \mathrm{w})$ 


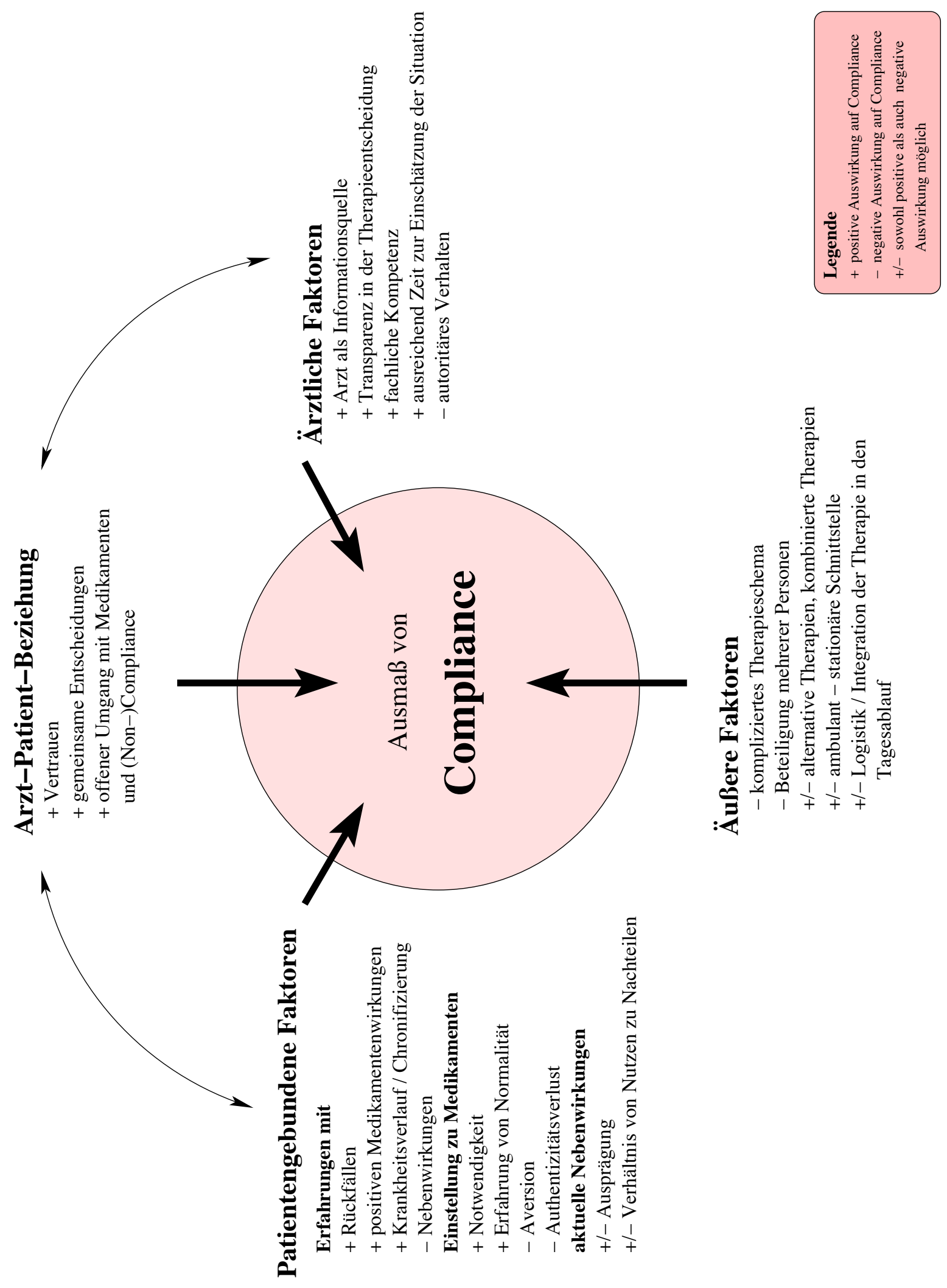

Abbildung 5.1: Einflussfaktoren auf Compliance 
P: [...] ich merk, dass ich das brauche, weil ich das sonst nicht aushalte. Ich halt es einfach nicht aus. [...] Im Grunde meines Herzens bin ich, glaube ich, stinknormal. (Q 17:27; 49 J., m)

[l:] ähm, wie stehen Sie selbst dazu? Also zu den Medikamenten?

$P$ : Ja, ich hab gemerkt, dass es mir gut tut. Ja, wenn ich die nehme, dann geht's mir, geht's mir gut, dann geht's mir ganz normal wie früher auch. Keine Beeinträchtigung oder so. (Q 5:51; $55 \mathrm{~J} ., \mathrm{w})$

Ebenfalls von Bedeutung war die Einstellung zu Medikamenten im Gesamtkontext der Therapie. Sie wurden von mehreren Patienten lediglich als ein Pfeiler einer erfolgreichen Therapie gesehen, in der jedoch auch andere Therapieformen, wie Psychotherapie, Gesprächstherapie, Gruppentherapie etc. einen wichtigen Platz einnahmen. Die Medikamente wurden dann insbesondere in der Akutsituation als notwendig eingeschätzt. Erst durch Sie wurden die anderen Therapieformen ermöglicht.

P: Dass halt zum Beispiel, äh, psychische Probleme halt doch fast immer mit Medikamenten behandelt werden und ansonsten nicht so sonderlich viel gemacht wird und halt häufig Therapien so als - - ja - - so als Schnick Schnack nebenbei angesehen werden. - - Medikamente berücksichtigen halt keine Lebensumstände und sonstige Sachen -also das ist - - das ist etwas schwierig. (Q 9:77; $26 \mathrm{~J} ., \mathrm{m})$

[P:] Es werden viel zu wenig Alternativmethoden zur Behandlung von Psychosen geboten. In erster Linie - - ist die Behandlung besteht aus Tablettenbasis - - um eine Nachbetreuung, eine ordentliche zu haben, müsste man eigentlich nach Psychologen oder, wie gesagt, gute Betreuer oder - - Anlaufstellen haben. (Q 19:17; 35 J., w)

[P:] Ich wende mal folgendes Beispiel an: Es kommt ein Patient in eine psychiatrische Klinik, der geistig sehr desorientiert ist und verwirrt ist, der tagelang nicht geschlafen hat und unter großer Angst leidet. In so einem Fall würde ich eine kurz- bis mittelfristige Verabreichung geeigneter Medikamente für ratsam halten, um das Leiden dieses Patienten zu beheben und die Bahn frei zu machen, den Weg frei zu machen, für eine psychotherapeutische Behandlung. Es gibt Medikamente, die Angst nehmen, wegnehmen, dass der Patient von der Angst befreit wird, dass er nachts wieder schläft und dass er wieder realitätsorientierter wird. Und da halte ich auch bei kurz; oder mittelfristiger Einnahme, halte ich den Schaden für äußerst geringfügig, aber den Nutzen für sehr groß. (Q 1:86; 52 J., m)

\subsubsection{Erfahrungen mit Medikamenten und Compliance}

Die eigenen Erfahrungen schienen im Bezug auf die Compliance des Patienten eines der wichtigsten Entscheidungskriterien zu sein. Generell konnten positive und negative Erfahrungen unterschieden werden, die sich auch entsprechend auf die Compliance auswirkten. Diese Erfahrungen machten Patienten in der Regel über einen längeren Zeitraum nach Beginn der Erkrankung, wobei sich ein Kreislauf von akutem Schub, Therapie, Noncompliance und erneutem akuten Schub in vielen Fällen mehrfach wiederholte. 
Machten die Patienten positive Erfahrungen mit der Medikamententherapie, so wirkte sich dies auch positiv auf ihre Schilderungen der weiteren Compliance aus. Hierzu zählte die Erfahrung, durch eine konsequente Einnahme der Medikamente kritische Situationen und Rückfälle verhindert zu haben. Hinzu kamen auch Erlebnisse, in denen der Patient nach dem Absetzen der Medikamente in eine kritische Situation geriet, die er aber selber durch erneutes Ansetzen abfangen konnte.

P: Also es ist jetzt nicht so, dass sie auf Medikamente drängt. Aber ich bin von mir aus, möchte ich auf kein_ auf die Medikamente nicht verzichten.

I: Warum?

P: Weil ich früher mal mitgemacht habe, da hatte mir der Psychiater gesagt, so jetzt nehmen sie keine mehr, Ihnen geht's gut. Vierteljahr später war ich wieder in der Klinik. Und das war nicht gut.

I: Hatten Sie die Medikamente auch schon mal selbst weggelassen?

P: Nein. (Q 5:52; 55 J., w)

P: Ja, ich hab das mal weggelassen. Ich hab gedacht, das Fluanxol lass ich mal weg und so.

I: Aber ganz komplett weggelassen?

P: Ganz komplett einfach weggelassen. Und jedenfalls und dann waren diese ganzen Eindrücke und Wirkungen und alles, das war auf einmal da, und da konnte ich überhaupt nichts mit anfangen und, wie gesagt, nee, da hab ich's auch schnell wieder genommen, ne. (Q 2: 66; $38 \mathrm{~J} ., \mathrm{m})$

[P:] Und das hab ich dann mal so 8 Tage lang durchgezogen, und da hab ich dann gemerkt, da fehlte was. Da fehlte tatsächlich die eine Tablette und ein Teil meines Gefühls der Einschätzung oder Wahrnehmung oder so, das war einfach -- schon anders und kaum auszuhalten. Das war natürlich eine ganz gute Erfahrung, dass ich schon gemerkt habe: 5 statt 6 oder 250 mg statt 300, das macht sich schon bemerkbar. (Q 17:28; 49 J., m)

Häufig wurde auch über Erfahrungen mit Rückfällen berichtet, die in der Regel unmittelbar mit dem eigenständigen Absetzen der Medikamente in Verbindung gebracht werden konnten. Diese negativen Erfahrungen wirkten sich dann positiv auf die weitere Compliance aus, indem sie im Anschluss eine konsequentere und zuverlässigere Einnahme der Medikamente zur Folge hatten.

[P:] Und darauf hin habe ich dann wieder diese Symptome bekommen und musste meine Ausbildung abbrechen. Da habe ich noch ein Jahr gar keine Medikamente mehr genommen - - wollte dann Fußball-Bundesliga spielen, bei der Eintracht Frankfurt, habe mich da angemeldet: Ich will bei euch mitspielen ((lachend)). Ja - - ich weiß jetzt halt, wie es wirkt, wenn ich die_ wenn ich mich dagegen wehre, gegen diese Tabletten. (Q 14:63; $36 \mathrm{~J} ., \mathrm{m})$

[P:] Und die erste Zeit der Erkrankung war das noch nicht so, dass man mir gesagt hat, es sollte mindestens 2 Jahre sein, und ich hab früher abgebrochen, und die Psychose 
ist immer wieder aufgetreten, und ich denke heute, es muss schon langfristig eingesetzt werden. (Q 18:26; 54 J., w)

Auch wurde von zwei Patientinnen der Verlauf der Erkrankung und Therapie direkt mit der Medikamenteneinnahme in Zusammenhang gebracht. So machte eine Patientin die Erfahrung, dass sich die Therapie für sich angenehmer und ärmer an Nebenwirkungen gestaltete, wenn sie kontinuierlich durchgeführt wurde. Eine weitere Patientin schrieb ihrer vorangegangenen Noncompliance die Chronifizierung der Erkrankung zu.

[P:] Also das muss man mindestens, bei mir zumindest, einrechnen, und wenn ich jetzt denke, ich bin wieder ohne Medikamente, die Psychose setzt wieder ein und ich muss zwei Jahre mich rumquälen, bis dass ich sage, ich bin einigermaßen nebenwirkungsfrei, dann bin ich bereit oder finde das in Ordnung, wenn ich jetzt eine ganz kleine Dosis kriege und mich wohl dabei fühle. (Q 18:31; 54 J., w)

[P:] Ach ja, ich habe mal in einem Bioladen gearbeitet und ich bin eigentlich so drauf, äh - - möglichst keine Chemie - - und deswegen habe ich anfangs auch die Medikamente weggelassen. Damit habe ich mir das vielleicht eingehandelt, dass sich die Krankheit chronifiziert hat. (Q 8:95; 38 J., w)

Negativ auf die Compliance wirkten sich vor allem Erfahrungen mit unangenehmen und beeinträchtigenden Nebenwirkungen aus. Diese hatten Angst vor weiteren medikamentösen Therapien zur Folge und konnte so zur Ablehnung oder Verweigerung der Einnahme eines Medikamentes führen. Diese Angst vor Nebenwirkungen, die auf persönlicher Erfahrung beruhte, ist von den aktuell während der Therapie auftretenden unerwünschten Wirkungen abzugrenzen Abschnitt 5.4.3.

[P:] Ich hab dann gesagt, ich probier das halt mal aus, und wenn die Nebenwirkungen zu krass sind, dann lass ich's halt einfach, egal was die Ärzte sagen (...). (Q 11:27; $23 \mathrm{~J} .$, w)

[P:] Da wurde ich eingeliefert und - - und der erste Psychiater, bei dem ich war, der fing dann auch mit Haldol an, oder was. Mag ja sein, dass das das Mittel ist, was man den Leuten halt gibt, halt irgendwie, wenn sie in den Phasen sind, aber ich hab das überhaupt nicht vertragen. Ich hab Krämpfe ohne Ende gekriegt, ja okay, gab's dieses Gegenmittel Akineton, aber das haben sie dann auch erst dann gespritzt, wo ich dann tagelang mit diesen Symptomen rumgelaufen bin und mich nicht bewegen konnte und so weiter. Und dann kamen die da oben im LKH dann auch auf einmal mit diesem Haldol an. Schwupp hier und Haldol, ne. Nee, nehm ich nicht. (Q 2:55; 38 J., m)

Ebenfalls negativ auf die Compliance wirkte sich der Eindruck aus, dass die Einnahme der Psychopharmaka keinen direkten Einfluss auf die Psychosymptomatik habe. In diesem Fall wurde kein Nutzen der Einnahme gesehen.

I: Und mit der Verwirtheit und mit den Stimmen, das ist dann nie wieder gekommen? P.: Ab und zu kommt es mal wieder, ne, aber denn geht's gleich wieder weg. Die Tabletten nehme [ich] denn nicht. Ich hab so Magentabletten, die nehme ich denne und dann geht's weg. (Q 15:11; 79 J., m) 
[P:] Aber - - ich hab nämlich bei mir selbst gemerkt, wenn ich gemerkt habe, ich bin nicht anständig betreut, es geht nicht aufwärts, und ich werde nur von den Medikamenten gehemmt, dann setze ich sie ab. Habe ich gemacht. (Q 19:21; $35 \mathrm{~J} ., \mathrm{w})$

\subsubsection{Auswirkungen von aktuell auftretenden Nebenwirkungen}

Neben den Erfahrungen mit unerwünschten Wirkungen der eingenommenen Medikamente beeinflussten auch die aktuell in der Therapie verspürten Nebenwirkungen die Compliance. Zusätzlich zu den körperlichen Nebenwirkungen wie Müdigkeit, Abgeschlagenheit, Gewichtszunahme und trockener Mund berichteten die Patienten über Wirkungen, die sie in ihrer Persönlichkeit beeinträchtigten. Sie fühlten sich abgeschirmt von sich selbst und ihrer Umwelt und erlebten einen Authentizitätsverlust. Auch ein vermindertes affektives Schwingungsvermögen wurde als belastend empfunden. Beide Formen der Nebenwirkungen hatten einen negativen Einfluss auf die Compliance.

\section{I: Was stört sie da?}

P: Ja, ich habe ja noch Angst_ich hab auch sehr zugenommen und so und manchmal fühle ich mich so müde und so zerschlagen, da habe ich auch immer das Gefühl, das kommt von den Medikamenten - - (Q 6:82; $36 \mathrm{~J} ., \mathrm{m})$

I: Es gab ja auch schon mal Zeiten, wo sie die Medikamente abgesetzt haben, selber. Können Sie sagen, warum das war?

$P$ : Ja, ich fand den Zustand einfach so schlimm. Weil eben überhaupt nichts war. Ich bin in so einer grauen Umgebung gewesen, und es kam überhaupt nichts an mich ran, es waren überhaupt keine Empfindungen mehr, --(Q 18:29; 54 J., w)

P: Ja, abgelehnt habe ich, weil ich damit einfach nicht klar kam, mit den Nebenwirkungen, mit dieser Unruhe innerlich, weil diese Spannungen, Muskelverspannungen. Oder, dass ich irgendwie keine Freude hatte - - [...] Weil ich durch diese Medikamente keine Glücksgefühle mehr hatte. (Q 14:62; $36 \mathrm{~J} ., \mathrm{m})$

Entscheidend für den Einfluss der Nebenwirkungen auf die Compliance war auch ihre Ausprägung. Es kam zu einer Abwägung des Verhältnisses von erwünschten zu unerwünschten Wirkungen. Hieraus ergab sich, dass wenig ausgeprägte oder beinträchtigende unerwünschte Wirkungen von den Befragten oftmals in Kauf genommen wurden in dem Wissen, damit den Alltag und weiteren Verlauf der Erkrankung positiv beeinflussen zu können.

P: Ich stehe dazu so, dass diese Medikamente mich von den Nebenwirkungen her kaum beeinträchtigen und dass sie mir einfach einen Schutz vor einer neuen Erkrankung geben.

I: Wie zufrieden sind Sie damit? Auch mit diesem Schutz? Also, das Medikament selbst, sind Sie damit recht zufrieden, auch zum Vergleich Nebenwirkung und Wirkung?

$P$ : Ja, bin ich sehr zufrieden. Meine Denkfähigkeit ist nicht eingeschränkt. Das sehe ich an der Arbeit, also, und ich fühle mich jetzt eigentlich so wie vorher, wie vor dem ersten 
Schub. Das ist schon was sehr Positives. Denn nach dem ersten Schub, als es mir noch nicht so gut ging, war ja noch gar nicht abzusehen, wie es weiterhin so, wie weit ich mich wieder rehabilitieren konnte. (Q 4:52; $30 \mathrm{~J} ., \mathrm{m})$

P: Ich hab gesagt, also dass ich zu viele Nebenwirkungen hab. Bei mir war es halt so, ich habe nicht mehr geschlafen. Ich hab schon mehrere Medikamente ausprobiert gehabt, zuletzt ein Forschungsmedikament, ein atypisches. Und bei mir haben die Schlaftabletten dann auch nicht mehr gewirkt. Und ich wollte halt noch arbeiten gehen und ich muss mich dann halt entscheiden. Rente einreichen, also quasi zu Hause bleiben mit den Medikamenten, oder Medikamente absetzten. Und dann habe ich mich halt für das Absetzen der Medikamente entschieden. (Q 19:29; 35 J., w)

\subsubsection{Informationen über Medikamente}

Das Ausmaß der dem Patienten zur Verfügung stehenden Informationen über ein Medikament hatte ebenfalls Einfluss auf die Compliance. Jedoch nicht nur der Inhalt, sondern auch Art und Umfang der Aufklärung schienen von Bedeutung zu sein, um die Verunsicherung möglichst gering zu halten. Als besonders wichtig beschrieben die Patienten den Informationsfluss in der therapeutischen Beziehung, da der Arzt für viele Patienten eine wichtige Informationsquelle bei gesundheitsrelevanten Fragen war. In der Medikamententherapie erwarteten sie von inm als Fachmann, dass er über das Medikament und seine Wirkungsweise aufklärte und auf etwaige Nebenwirkungen vorbereitete. Das Medikament verlor seine Bedrohlichkeit, wenn der Patient im Vorfeld wusste, was auf ihn zukommen konnte. Auch alternative und ergänzende Methoden sollten in der Beratung berücksichtigt werden. Der Aspekt des Informationsflusses spielte ebenfalls für die Ausbildung einer tragfähigen therapeutischen Beziehung eine relevante Rolle.

I: Und wenn Sie z. B. selbst Ärztin wären und würden einer Patientin z. B. das Leponex empfehlen, was wäre Ihnen dann wichtig zu sagen?

$P$ : Ja, die Nebenwirkungen eben. Was, was furchtbar ist Mund_ man hat immer Durst, man hat - - - na ja und es macht etwas müde. (Q 5:28; $55 \mathrm{~J} .$, w)

[P:] Dass man über Nebenwirkungen aufgeklärt wird, weil viele Hausärzte sagen, ja wenn ich meinen Patienten die ganzen Nebenwirkungen nun noch erkläre, dann sitzt er drei Tage später bei mir in der Praxis und hat von den Nebenwirkungen fünfzehn. Und ich denke, wenn man da--offen irgendwie dran herangehen würde, dann wäre das auch nicht der Fall. (Q 9:61; 26 J., m)

\subsubsection{Güte der Arzt-Patient-Beziehung}

Mehrere Patienten dieser Untersuchung schätzen eine gute, vertrauensvolle Beziehung zum Arzt als förderlich für die Therapie und die Compliance ein. 
$P$ : Ich überlege, ob zwischen dem zweiten und dritten Schub, - - da war auch noch so eine Phase, wo ich zeitweise etwas weniger genommen hatte als von ärztlicher Seite empfohlen. Also war es quasi so, dass sich das Vertrauensverhältnis wirklich so nach und nach erst - - verbessert hat. (Q 4:25; $30 \mathrm{~J} ., \mathrm{m})$

P: Ich konnte von ihm das auch annehmen, was ich vielleicht von wem anders nicht angenommen hätte. Weil wir diese gute Beziehung hatten_, oder haben, habe ich dann auch angenommen, wenn er sagt: Ja, aber Frau XXX, aber wir müssen das jetzt erst mal machen. Also der Ton macht ja oft die Musik, ne. Und wenn mir ein anderer Arzt die gleichen Worte gesagt hätte in einem anderen Tonfall, dann hätte ich sofort so eine - eine Abneigung entwickelt, jetzt dann auch wirklich dann die Sache zu nehmen. Aber bei meinem Arzt hatte ich das Gefühl, dass er einfach da auch appelliert hat an meine Einsicht, ne. [...] (Q 8:47; 38 J., w)

Im Gegensatz dazu, wurde ein wenig vertrauensvolles Verhältnis zum Arzt als eher hinderlich für die Compliance gesehen, wie ein Patient an einem konkreten Beispiel verdeutlichte:

P: Also ich bin da eher immer ein bisschen misstrauisch, wenn er mir dann irgendwelche Medikamente gibt so. Also ich war das letzte Mal wegen einer Erkältung da und da hatte ich dann irgendwie den Eindruck, das was er mir gegeben hatte, das war ein Placebo, und da hab ich mich dann so ein bisschen geärgert, dass er da überhaupt keine Skrupel hatte mit einem Placebo das Geld aus der Tasche zu ziehen. Und ich habe dann hinterher noch mal drüber nachgedacht und dann dachte ich, dass es wahrscheinlich doch kein Placebo war, aber so richtig vertrauen tue ich ihm eigentlich nicht. (Q 21:4; $22 \mathrm{~J} ., \mathrm{w})$

\subsubsection{Therapeutische Entscheidungen}

Ein wesentlicher Prozess in einer Arzt-Patient-Beziehung ist die Entscheidungsfindung bezüglich der Therapie und damit auch der Medikamente. Sie kann auf einem kontinuierlichen Spektrum von einer fast autonomen Gestaltung des Patienten bis hin zu einem ausgeprägt autoritären Führungsstil des Arztes unterschiedliche Ausprägungen haben.

So erlebten es die meisten Patienten als positiv, wenn Entscheidungen im Dialog gefällt wurden. Sie wollten die Möglichkeit haben, Einfluss zu nehmen, und es fiel innen leichter, die Therapie einzuhalten, wenn sie auch persönlich hinter der Entscheidung stehen konnten. Sie hielten es für wichtig, eigene Verantwortung zu übernehmen, zum Beispiel bei der Anpassung der Reservemedikation.

[P:] Wir arbeiten regelrecht zusammen. Sie fragt mich, wie es mir geht, und ich sag, mir geht es heute nicht so gut. Dann sagt sie zu mir, vielleicht werden wir die Medikamente ein bisschen erhöhen, vielleicht geht es ihnen dann besser. Oder wir lassen etwas weg, damit es ihnen besser geht. (Q 5:10; $55 \mathrm{~J} ., \mathrm{w})$

[P:] Also, weil er ja auch gespürt hat, wenn das jetzt absolut gegen meinen Willen geht, dass das vielleicht auch nichts bringt, ne. - - Also, ne, dadurch habe ich schon das_- ja, genau, vielleicht ist es das, was Sie gerade sagen, ich habe nicht das Gefühl gehabt, mir wird was übergestülpt, ne - - [...] (Q 8:47; $38 \mathrm{~J} .$, w) 
I: Und können Sie drauf Einfluss nehmen, was jetzt verschrieben wird oder welche Dosis? Können Sie das gleichberechtigt besprechen?

$P$ : Ja, also schon. Also, ich habe schon, schon etwas dafür gekämpft, dass es runtergesetzt wird und kann auch, kann auch, wenn es mir mal nicht so gut gehen sollte oder so, nehme ich auch zwischendurch mal eine oder am Tag oder so. Also das kann ich schon machen. (Q 23:25; 41 J., m)

Eine der extremen Ausprägungen des oben angeführten Kontinuums ist eine weitgehende Autonomie des Patienten, der sich die alleinige Entscheidung vorbehält. Diese Position ist allerdings zu relativieren, da im Prozess der Entscheidungsfindung der Arzt als Experte auf dem Gebiet durch Filterung der Information und lenkende Ratschläge Einfluss nimmt. Der tatsächliche Einfluss dürfte jedoch sehr variabel und schwer zu erfassen sein. Einige Patienten gaben ganz bewusst an, dass sie den Arzt bei der Medikamententherapie als Ratgeber sehen, der sie lenke, jedoch nicht bestimme. Vielmehr sprachen sie vor allem sich selber die Kompetenz zu, einzuschätzen, was gut für sie sei und behielten sich daher die letztendliche Entscheidung vor.

[P:] So ein wirklich richtiges Mitspracherecht gab es nicht. - - Ich habe natürlich selber zu entscheiden, ob ich das nehme oder nicht, die können mich ja nicht dazu zwingen, (...) Aber sie lassen auch nicht locker wenn die jetzt meinen, dass man das nehmen sollte, dann lassen die einen auch damit nicht in Ruhe. (Q 11:26; $23 \mathrm{J.}, \mathrm{w})$

P: Da war ich schon beim Arzt, das ist lange her, da sage ich, ich hätte gern die Spritzen, da sagt er, wieso denn. Da sage ich, ich brauch das, mir geht's nicht gut. Ich mein, der hat es mir dann nicht angesehen. Ich mein, es gibt vielleicht Leute, den sieht man das schon grad förmlich an. Aber ich meine, bei mir war es eben nicht so, dass es nicht ging. Also muss ich das doch wissen, und ich weiss das denn schon.

I: Also Sie merken selber, was Sie brauchen und Sie nehmen das auch ein?

$P$ : Ja, ich will das denn nehmen. Und dafür mach ich das denn auch. Das ist klar und da geh ich nicht zum Arzt und mach mal und frag den, ob das, also das ist ja Quatsch. (Q 17:29; 49 J., m)

Am anderen Ende des Spektrums ist es der Arzt, der die Entscheidungen bezüglich der Medikamente trifft und sie ggf. auch gegen den Patienten durchsetzt oder dies zumindest versucht. Dieses Extrem wurde von den Patienten als negativ wahrgenommen. Zudem empfanden sie den Arzt als Autorität, der man sich nur schwer entziehen oder widersetzen könne, was bis hin zu dem Gefühl des Ausgeliefertseins führen konnte. Ein mäßig ausgeprägter autoritärer Führungsstil des Arztes hingegen wurde durchaus begrüßt und in bestimmten Situationen als notwendig erachtet. So schien insbesondere in der akuten Erkrankungsphase eine „starke Hand“ maßgeblich zum Erfolg der Therapie beitragen zu können, da der Patient in dem Moment krankheitsbedingt auf klare Richtlinien angewiesen sei. Jedoch auch in der langfristigen Therapie war einigen Patienten ein eher patriarchalischer Stil des Arztes angenehm, solange dessen Entscheidungen transparent waren und dem Patienten nachvollziehbar blieben. 
[P:] Und auch während der stationären Behandlung ist man natürlich dann an die Weisungen der Ärzte dort gebunden, wenn man keine nachteiligen Folgen für sich in Kauf nehmen will. (Q 4:47; $30 \mathrm{~J} ., \mathrm{m})$

I: Also, wenn ich das richtig verstehe, würden Sie es so beschreiben, dass Sie in der akuten Phase - - eine klarere Führung brauchen?

P: Ja, denk ich schon, denk ich schon. Also Frau F. hat das sehr bestimmt gemacht, und aber immer noch freundlich und hat gesagt, wir machen das jetzt einfach mal. Wir machen ein Depot, das ist einfach besser und bei Depot kann man sich ja nicht rausschleichen und rausschummeln und die Depotspritze, die ist die Grundlage gewesen, dass ich auch Risperdal regelmäßig genommen habe und jetzt eben seit sechs Jahren gut, ich bin seit sechs Jahren in ambulanter Behandlung und nehme auch freiwillig meine Risperdal-Tabletten. (Q 18:15; 54 J., w)

[P:] Ja und ich meine am Anfang oder in der ersten Hälfte der Zeit war einfach ein bisschen mehr auch Druck von den Therapeuten da, dass sie halt gesagt hatten, wenn ich die nicht nehme, dann werde ich quasi zum Frührentner, verliere ich meine Arbeitsstelle. Ich kann jetzt nicht mehr sagen, ob das falsch oder richtig von ihnen war, denn es kam ja darauf an, auch mir das deutlich zu machen, und manchmal sind halt deutliche Worte auch mal notwendig. (Q 4:28; $30 \mathrm{~J} ., \mathrm{m})$

\subsubsection{Umgang mit Noncompliance in der therapeutischen Beziehung}

Die befragten Patienten schilderten zwei grundsätzliche Möglichkeiten, im Rahmen der therapeutischen Beziehung mit Noncompliance umzugehen: man könne sie thematisieren oder nicht. Wurde sie zum Thema, so berichteten die Patienten wiederum eine Bandbreite an möglichen Reaktionen auf Seiten des Arztes, die von Verständnis und Dialog bis hin zu Sanktionen reichten.

Sprachen Patienten in der Sprechstunde Noncompliance nicht an, so lag das zum Teil daran, dass sie sich nicht trauten, ihr Verhalten einzugestehen. Obwohl man bei Noncompliance nicht objektiv von Unrecht sprechen kann, war dennoch häufig ein unterschiedlich ausgeprägtes Unrechtsempfinden vorhanden. Oftmals jedoch fielen Noncompliance und der Abbruch des therapeutischen Kontakts zusammen, so dass ein Dialog nicht stattfinden konnte.

P: Ja, dazu fällt mir ein, dass nach dem ersten Schub und nach der Nachbehandlung dann eine Zeit war, wo ich die Medikation halt mal abgesetzt habe, ohne das dem Psychologen zu sagen, weil ich mich da einfach nicht getraut hatte, die Wahrheit zu sagen. Und ja, ich sehe das halt in der Nachschau halt als Fehler ein. Und das war halt schon ein Konfliktpotential, weil man nicht genau ehrlich sein konnte und hier und da lügen musste, was ich eigentlich nicht gerne mache. Aber ich hatte zu der Zeit mich nicht anders zu verhalten gewusst. (Q 4:35; $30 \mathrm{~J} ., \mathrm{m})$

I: Als Sie mal diese Medikamente abgesetzt haben, konnten Sie da mit Ihren Ärzten drüber sprechen? 
P: Nein.

I: Warum nicht?

P: Ja, das ist so dieser Zwiespalt gewesen. Einmal hab ich gedacht, ich kann das selbst steuern und auf der anderen Seite war mir schon bewusst, dass das nicht in Ordnung gewesen ist, was ich gemacht habe. Aber das Unrechtsbewusstsein ist nur ganz wenig ausgeprägt gewesen. Aber ich hab es, ich hätte vielleicht drüber sprechen können, aber ich wollte auch nicht drüber sprechen. Ich wollte auch nicht drüber sprechen und war eigentlich für mich und -- kein großartiges schönes Tun, was ich da gemacht habe. Also das Unrechtsbewusstsein war ziemlich gering. (Q 18:34; 54 J., w)

I: Haben Sie dann auch mal mit Ihrem Arzt darüber gesprochen, dass Sie die Medikamente abgesetzt haben?

P: Nee, ich hatte, als ich diese Tabletten abgesetzt habe, bei der Post, da habe ich es ihm gesagt, dem Herrn XXX: Ich nehme diese Spritze nicht mehr. Aber als ich die dann nicht mehr hatte, da war ich auch_ bin ich gar nicht mehr zum Nervenarzt gegangen. (Q 14:69; $36 \mathrm{~J} ., \mathrm{m})$

Wurde in der therapeutischen Beziehung Noncompliance thematisiert, so empfanden die interviewten Patienten es in erster Linie als positiv, wenn der Arzt im Gespräch versuchte, ihr Verhalten zu verstehen und die Therapie entsprechend ihrer Bedürfnisse zu modifizieren. Eine Patientin äußerte die Idee, dass der Arzt seine Patienten in der Erfahrung mit Noncompliance unterstützen solle, statt diese forciert verhindern zu wollen.

P: Ja, das war so. Mit dieser Spritze, das war so im August 1995, da war ich vorher ein halbes Jahr im Krankenhaus gewesen. Und ich hatte manchmal meine Medikamente nicht so regelmäßig genommen, wenn ich zu Hause war, oder so. Das ist richtig aufgefallen, dass es mir auch manchmal schlechter ging. - - Und da hatte der leitende Arzt gesagt, es wäre besser, also bei der Visite, mir das depotmäßig zu geben, halt mit einer Spritze, damit ich nicht mehr dran denken muss und es halt im Körper schon habe. War auch dann einsichtig und hab ich das auch verstanden, hab's dann auch mitgemacht, [...] (Q 6:44; $36 \mathrm{~J} ., \mathrm{m})$

[P: ...] also dass er das auch einsieht, dass man z. B. Nebenwirkungen zeigt und -auch den Versuch macht, mit Kontrolle die Tablette noch mal zu reduzieren. - - Dass also wichtig ist, da natürlich, dass da die Betreuung da ist, weil der Patient setzt die Tabletten so oder so ab, und dann sollte der Arzt wenigstens sagen: Okay, dann aber mit meiner Hilfe und mehreren Besuchen in kürzeren Abständen. Und dann geht das auch ohne Medikamente oder mit weniger. (Q 19:23; 35 J., w)

Als negativ hingegen wurde wahrgenommen, wenn der Arzt mit Tadel oder Sanktionen auf das Verhalten des Patienten reagierte oder sogar seinerseits die therapeutische Beziehung abbrach.

P: Ja. Ja, das kam auch, weil ich das, was er mir verschrieben hatte, dann nicht genommen habe. 
I: Haben sie ihm das gesagt?

P: Ja, hab ich ihm dann gesagt.

I: Was ist dann passiert?

$P$ : Ja, meinte er, warum ich dann zu ihm hinkomme. Bräuchte ich doch gar nicht mehr zu ihm hingehen und fertig aus. Ja und dann hat er dann noch gesagt zu der Sprechstundenhilfe, bräuchte ich ja da nicht mehr hinkommen. (Q 2:14; $38 \mathrm{~J} ., \mathrm{m})$

\subsection{8 Ärztliche Faktoren}

Neben den Einstellungen und Erfahrungen der Patienten sowie ihrer Beziehung zum Arzt waren für die Compliance auch Faktoren wichtig, die in erster Linie auf den Arzt und sein Verhalten zurückzuführen waren. So spielte etwa die ärztliche Kompetenz eine wichtige Rolle. Entscheidend schien hierbei auch zu sein, inwiefern die Erwartungen an den Arzt und sein tatsächliches Verhalten divergierten. (Es sei hier angemerkt, dass in dieser Teilstudie keine Daten zur tatsächlichen Kompetenz der Ärzte erfasst wurden. Es handelt sich also in den folgenden Aussagen um Einschätzungen der Patienten.)

[P:] Also wenn man dann nur einmal halt in irgendein psychiatrisches Lehrbuch reingeschaut hat und dann da gelesen hat, ja, wenn die Leute sich jetzt irgendwie für den Papst halten oder so was, dann - - muss man halt einen Krankenwagen holen, die Leute wegbringen lassen, -- und da habe ich schon manchmal den Eindruck, dass das Wissen vieler Hausärzte so ähnlich bestellt ist darum: Und das ist halt ein bisschen dürftig dann doch. - - (Q 9:95; 26 J., m)

P: [...] Der ist schon ganz nett, aber das jetzt mit der Psychiatrie hat er überhaupt nicht verstanden. Also da meinte er auch nachher, als er diese Zettel da halt bekommen hat, wo halt drauf stand irgendwie, welche Medikation ich habe und was das für eine Diagnose ist. [...] aber das vielleicht trotzdem zu locker nimmt alles. Er hat z. B. auch damals zu meinem Abitur eine Packung ziemlich starke Schlaftabletten gegeben, und ich denk halt im Nachhinein, dass es unvorsichtig von ihm war, weil ich da halt auch ein paar Monate später die ganze Packung genommen habe. (Q 21:3; 22 J., w)

P: Sie hat ja gar nichts gemacht! Sie hat mich angeguckt, hat mich gefragt, wie es mir ginge, und da hab ich ihm das gesagt, dem Psychiater nech, also, da sagt er: Nein, ich verschreibe keine Tabletten. Sie müssen da hier ins $\mathrm{Kr}_{-}$, ins_ ins Dings gehen, da, - ins Heim gehen.

I: Und die hat gar keine Tabletten verschrieben?

P: Nein, gar nicht! (Q 15:2; 79 J., m)

Ebenso wichtig für die Compliance war aber auch das Verhalten des Arztes in der Situation, in der er eine Therapie vorschlägt oder anordnet. Es war den befragten Patienten wichtig, dass er vor der Medikamentenanpassung ein ausführliches Gespräch führte und sich Zeit nahm, den aktuellen Zustand des Patienten möglichst vollständig zu erfassen. War dies nicht der Fall, sondern erfolgte eine von dem Patienten als voreilig eingeschätzte Anpassung, stellte er die ärztliche Entscheidung in Frage. 
P: Ja, mir kam das so vor, als wenn er über mich besser Bescheid wüsste, wie ich über mich hier, obwohl ich mit ihm gar nicht groß gesprochen habe. Ich mein, mag ja alles sein, aber das hilft mir auch nicht weiter, wenn er mich da weiter mit irgendwelchen Medikamenten wieder voll stopft da. (Q 2:49; 38 J., m)

[P:] Das war mir eine Zeit lang sehr wichtig bei meinem Hausarzt, dass er nicht sofort mit Medikamenten losschießt, sondern erstmal guckt, ist es wirklich eine gefährliche Situation, weil wenn man mit so einer Krankheit zu kämpfen hat, gerade, äh - - wenn man da noch dabei ist, zu lernen damit umzugehen. (Q 8:75; $38 \mathrm{~J} ., \mathrm{w})$

\subsection{9 Äußere Einflüsse}

Neben den personen- oder beziehungsgebundenen Einflussfaktoren auf Compliance spielten auch äußere Faktoren eine Rolle. Zu ihnen zählten zum Beispiel komplizierte Therapiepläne oder Alltagsabläufe, aufgrund derer der Patient die Einnahme vergaß. Außerdem war es im gesamten therapeutischen Gefüge, in dem mehrere Personen beteiligt und die Zuständigkeiten nicht klar abgegrenzt waren, zu Schwierigkeiten und Unsicherheiten gekommen. Diese äußeren Einflüsse schilderten die Patienten allerdings als eher zweitrangig für die Compliance.

[P:] So ein bisschen diszipliniert muss man ja da schon sein, immer zur gleichen Zeit die Getränke besorgen auch. --

I: Sind das eher so praktische Gründe, warum sie das

P: Ja. Also eigentlich schon, ja. Ich trau mir da auch nicht so über den Weg manchmal, denk dann auch manchmal: Ja da machst du es dann doch nicht, und dann geht's dir wieder schlecht, ne.

I: Weil sie es schlicht dann manch mal auch vergessen hatten im Alltag, oder?

P: Ja. Ja, das geht schnell mal. Wenn du mal unterwegs bist oder so, hast auch gar nicht die Gelegenheit, was zu trinken oder so irgendwie. Das ist alles ein bisschen komisch. $--(\mathrm{Q} 6: 87 ; 36 \mathrm{~J} ., \mathrm{m})$

[P:] Und da ging es irgendwie nicht und was ich da vom Krankenhaus gekriegt habe da, diese Tropfen. Und da sagt er, was haben Sie denn da? Alle haben was anderes gesagt, wenn was war. Sag ich, ja hier vom Krankenhaus. Um Gottes Willen. Schmeißen sie die weg, oder schmeißen sie die Dr. XXX vor die Füße. Es soll ja besser werden und nicht schlechter, ist aber schlimmer geworden. Und dann hat er ne Spritze gekriegt - - ein paar Minuten, da war es okay. Da war es vorbei. [...] (Q 15:9; 79 J., m)

\subsection{Einflussfaktoren einer guten Arzt-Patient-Beziehung}

Bereits in Abschnitt 5.4.5 wurde betont, welchen wesentlichen Einfluss eine gute Arzt-PatientBeziehung auf die Compliance hat. In diesem Zusammenhang werden auch die Faktoren interessant, die zur guten Qualität der Arzt-Patient-Beziehung beitragen. Genannt wurden 
in den Interviews vor allem (1) strukturelle Merkmale, (2) die Passung zwischen Arzt und Patient und (3) therapeutische Merkmale.

Strukturelle Merkmale einer Beziehung sind durch äußere Umstände vorgegeben und spielen sich nicht auf einer persönlichen bzw. zwischenmenschlichen Ebene ab. Hierzu gehören die Dauer der therapeutischen Beziehung, die Häufigkeit und Dauer der einzelnen Kontakte und die Infrastruktur der Praxis. Unter dem Aspekt der Passung zwischen Patient und Arzt lassen sich Aussagen von Patienten subsummieren, die sich auf einer zwischenmenschlichen Ebene zwischen den beiden Personen abspielen. Dazu zählen der persönliche, über das Professionelle hinausgehende Kontakt, weiterhin Faktoren auf emotionaler Ebene, wie Empathie, Sympathien und Antipathien (die „Chemie“), ebenso ideologische Übereinstimmungen und Beziehungsaspekte, wie zwischenmenschlicher Respekt und Autonomie. Neben den persönlichen Kompetenzen des Arztes ist auch seine fachliche und therapeutischen Kompetenz wichtig, um ein vertrauensvolles Verhältnis aufzubauen.

\subsubsection{Dauer und Kontakthäufigkeit der Beziehung}

Die Dauer der Beziehung trug bei den befragten Patienten insofern zur Güte der Beziehung bei, dass der Arzt den Patienten im Laufe der Zeit zunehmend kennenlernte und auch seine privaten Lebensumstände und die psychiatrische Erkrankung besser einzuschätzen vermochte. Ebenso beschrieben die Patienten auch einen Effekt des sich Aneinandergewöhnens über eine lange Beziehungsdauer, so dass man mit den Eigenarten des Gegenübers umzugehen lerne. Als positiv erlebt wurde es außerdem, wenn der Hausarzt schon vor Ausbruch der psychiatrischen Erkrankung bekannt war und bereits eine Vertrauensgrundlage bestand.

[1:] Was muss z. B. so ein Arzt an sich haben, damit Sie ihm vertrauen können? $P$ : Das Vertrauen kommt immer erst, wenn ich einen länger kenne und--auch wenn er nett ist und ein bisschen freundlich (...) (Q 16:7; $50 \mathrm{J.}$, w)

$P$ : Ja, also da ich da schon recht lange in Behandlung bin, kennt man sich eigentlich auch ganz gut. Also das ist - - ja, wie beschreibt man so was? - - - Das ist schon natürlich eine Arzt-Patient-Beziehung, aber der weiß halt natürlich über mein Leben und meine Lebenssituation Bescheid, also in den Maßen -- wie es auch so sein sollte, sage ich mal - - wie mir das auch angenehm ist. (Q 9:2; $26 \mathrm{J.}, \mathrm{m})$

Eine Besonderheit trat bei der Beziehung zum Facharzt auf. Zwar spielte auch hier die Dauer der Beziehung eine Rolle, wichtiger war jedoch die Art des Kontakts. Als „Seelenarzt“ mit der fachlichen Ausrichtung auf die Psyche und mit Zeit für Gespräche konnte bei ihm eine tiefer gehende Beziehungsebene erreicht werden.

P: Den Psychiater kenne ich besser, da kann man halt auch mehr erzählen. Weil man sich halt auch recht häufig gesehen hat und auch über einen längeren Zeitraum, und es ist ja auch so, dass halt die Gespräche mit dem Psychiater etwas länger sind, als die beim Hausarzt. (Q 9:90; 26 J., m) 
Als negativ wurden kurzdauernde therapeutische Beziehungen wahrgenommen. Hier war es oftmals (noch) nicht möglich, intime Themen anzusprechen, insbesondere auch Aspekte der psychischen Erkrankung.

P: - - Ja, wie gesagt, das ist jetzt schon wieder ein neuer Arzt, und das ist dann immer

- - kann man auch erst man schlecht einschätzen [...] (Q 2:51; $38 \mathrm{~J} ., \mathrm{m})$

P: --, ähm, bisher habe ich, also den Hausarzt kenn ich noch gar nicht so lange, erst ein bis zwei Jahre, und, äh, bisher habe ich über meine psychischen Probleme mit ihm eigentlich fast gar nicht gesprochen, ganz wenig nur. (Q 1:91; $52 \mathrm{~J} ., \mathrm{m})$

Für die Patienten gab es zudem einen Zusammenhang zwischen häufigem Kontakt und der Ausbildung einer vertrauten Beziehung. Im Gegensatz dazu wurde das Verhältnis als weniger vertrauensvoll eingeschätzt, wenn lediglich ein unregelmäßiger, ausschließlich am aktuellen Bedarf orientierter Kontakt bestand.

P: [...] Aber im Rückblick muss ich sagen, war es auch - ja, was Sie sagten schon, so eine Regelmäßigkeit, ne. Also, zu wissen, ich bin nicht allein mit meinem_- - mit dieser Krankheitsgeschichte und äh - - - sondern das trägt irgend jemand so ein bisschen mit, ne - - Und das kann ich wirklich sagen, so habe ich das empfunden bei ihm, ne. (Q 8:60; $38 \mathrm{~J} ., \mathrm{w})$

[P:] Also, man könnte ihm auch alles erzählen, im Grunde genommen, dem Hausarzt. Aber das passiert halt eben nicht, da ich eben zu diesem Psychologen gehe halt, ne. Und doch selten dem Hausarzt. (Q 23:3; 41 J., m)

Neben der Häufigkeit des Kontakts spielte aber auch die Dauer des einzelnen Kontakts eine Rolle bei der Ausbildung einer guten Arzt-Patient-Beziehung. Eine große Anzahl von Interviewpartnern betonte, wie wichtig es ihnen sei, dass sich der Arzt Zeit für Gespräche nehme. Dies galt speziell bei besonderem Bedarf, wie etwa kritischen Situationen im Krankheitsverlauf oder außergewöhnlichen persönlichen Belastungen.

P: Ja, dass sie richtig über meinen Vater mit mir gesprochen hat. Das hat mir am allermeisten gefallen. Dass sie, das sie auch noch Zeit hatte in dem Moment, obwohl es schon nach Feierabend war. Ja. (Q 5:54; 55 J., w)

P: Sie ist präsent und die Probleme sind präsent und wenn ich ein größeres Problem hätte, dann könnte ich das durchaus mit ihr ansprechen und dann hat sie sofort Zeit und macht sich einfach frei von irgendwelchen Verpflichtungen und denn sagt sie, jetzt ist einfach Zeit dafür. Und das finde ich sehr positiv. (Q 18:39; 54 J., w)

Dem gegenüber erlebten es die Patienten sowohl auf der persönlichen als auch der fachlichen Ebene als negativ, wenn der Arzt spürbar unter Zeitdruck stand. So herrschte die Meinung vor, dass insbesondere der Hausarzt zu wenig Zeit für die Betreuung psychischer Erkrankungen habe. 
$P:$ Ja, das ist immer sehr kurz. Ich trau mich auch schon gar nicht so viel zu fragen, weil ich denk, der hat ja jetzt auch genug zu tun und draußen warten schon wieder fünf, sechs Leute, und - - ich meine, - - ich hab schon manchmal das Bedürfnis, mit ihm bisschen mehr noch zu reden. Ich trau mich dann aber auch nicht so - - (Q 6:9; $36 \mathrm{~J} ., \mathrm{m})$

P: Weil, ich hab es nämlich auch schon mal erlebt, dass man hingegangen ist und bei manchen Ärzten zu Hause schon formulieren musste, was man wollte. Da bin ich mit einem Zettel hingegangen und hab mir alles aufgeschrieben, damit ich alles sagen konnte, bevor der nächste kam. Das muss ja nicht sein. (Q 19:37; 35 J., w)

\subsubsection{Infrastruktur}

Auch infrastrukturelle Aspekte wurden in den Interviews immer wieder erwähnt. Hierzu zählten die Erreichbarkeit des Arztes, sowohl bezüglich der Lage der Praxis als auch der Sprechzeiten, außerdem die Organisation von Notdiensten und Urlaubsvertretungen. Sie standen zwar nicht unbedingt primär mit der Güte der Arzt-Patient-Beziehung im Zusammenhang, spielten jedoch eine Rolle bei der Wahl des Arztes bzw. der Praxis. So hielt eine Reihe von Patienten die geographische Nähe und verkehrsgünstige Lage der Praxis für wichtig.

P: Es ist schon mal wichtig, dass es nicht sehr weit weg ist von zu Hause. Das ist schon mal sehr wichtig. Ja wenn ich jetzt woanders hinziehen würde, dann müsste ich mir ja einen suchen. Da wüsste ich nicht, wie - - Ich würde immer in der Nähe jemanden suchen. (Q 5:49; 55 J., w)

P: Die hab ich dann gewechselt. Ich hab dann halt überall geguckt, wer hat die besten Öffnungszeiten, und damals hatte ich eine gefragt, die ich noch relativ gut erreichen kann wenn ich von Frankfurt heim komme. Und das war die einzigste, die in der Nähe vom Bahnhof war und die abends noch geöffnet hat. Das war eigentlich die Entscheidung. (Q 19:7; 35 J., w)

Außerdem wurde es positiv bewertet, wenn der Arzt von den Sprechzeiten her kurzfristig und unkompliziert zur Verfügung stehen konnte, insbesondere auch in Notsituationen. $\mathrm{Er}$ sollte Hausbesuche machen und eine gut informierte und erreichbare Urlaubsvertretung organisieren.

[P:] ich habe die Möglichkeit, wenn einer eben im Urlaub ist, dann kann ich in dieselbe Praxis gehen, weil die einen Assistenten haben. Die haben auch samstags und so und bis Mittag_irgendwie ist das ganz gut. (Q 17:10; 49 J., m)

[P:] es sollten auch von den Hausärzten mehr stützende Gespräche, - - äh, die auch bei Hausbesuchen stattfinden können, es sollte auch mal ein Hausarzt bereit sein, bei einer psychischen Erkrankung, wie ich es jetzt erlebe bei einer Mitbewohnerin in der WG, der Hausarzt auch mal einen Hausbesuch machen. (Q 1:96; 52 J., m)

[P:] Ich müsste irgend jemanden haben, zu dem ich ganz schnell kann, weil solche Phasen können ja ganz plötzlich kommen, und dann kann ich auch nicht noch drei Stunden im Wartezimmer sitzen oder so lange auf einen Termin warten (Q 8:5; 38 J., w) 


\subsubsection{Persönlichkeit und Kompetenz des Arztes}

Unter dem Aspekt „Persönlichkeit des Arztes“ werden Äußerungen der Patienten über die Arzt-Patient-Beziehung zusammengefasst, die eine persönliche Beziehung ermöglichten und über einen rein professionellen, auf die Medizin beschränkten Kontakt hinaus gingen. So erlebten es die Patienten als positiv, wenn der Arzt innen gegenüber aufgeschlossen war, sich für ihre private Situation interessierte und darauf einging. Es sollte eine Atmosphäre von Gleichberechtigung und Normalität herrschen. Für einige Patienten wurde der Arzt so auch Ansprechpartner und Ratgeber bei Problemen im Alltag.

$P:$ Ja, ich hab das zwar nie gemacht, mit ihm über persönliche Probleme gesprochen oder so, aber ich finde, er könnte einfach mal fragen, wie es sonst alles so vorginge in Ihrem Umfeld, oder haben Sie irgendwelche größeren Probleme, das könnte er ja auch mal fragen. (Q 11:44; $23 \mathrm{~J} ., \mathrm{w})$

[P:] Klar muss ich mit so einem Arzt auch reden können und natürlich auch [...] versuchen, über Probleme zu sprechen und anzuschneiden oder, Möglichkeiten, Rat und Hilfe zu erfahren, was kann ich denn jetzt eventuell woanders für mich denn besser lösen, wenn es schon in dieser Praxis nicht geht. Aus was für Gründen auch immer. Aber muss da wenigstens drüber sprechen können. Also so ein Arzt, der muss doch darüber hinaus selbstverständlich auch mal zuhören können. (Q 17:18; 49 J., m)

P: Ja. - - Ich brauche das auch manchmal, dass man mir so Tipps gibt, ne. Also, -so Schema, wonach man handeln kann. - - Ich weiß nicht, wenn man im Sport weit kommen will, braucht man auch einen Trainer, der einem sagt: Mach mal das, mach mal das. (Q 14:46; 36 J., m)

Für die Patienten war es nicht nur wichtig, dass sie dem Arzt vertrauen konnten, sondern ebenso, dass er innen Vertrauen entgegen brachte. Am Anfang der Beziehung war es daher nötig, einen Vertrauensvorschuss zu geben. Außerdem förderte es die Bildung einer persönlichen Beziehung, wenn man Gemeinsamkeiten entdeckte.

[P:] Oder sie haben mich zum Beispiel gefragt, ob ich irgendwelche Drogen genommen hätte oder Haschisch geraucht hätte. Bloß wenn ich dann sage „Nein.", dass es dann auch gut ist, und es wird ja sowieso kontrolliert, und dass sie das dann noch dreimal nachfragt oder so ungefähr. (...) dass die mir halt glauben können, (...) mir das Vertrauen halt auch geben können. (Q 11:9; $23 \mathrm{~J} .$, w)

[P:] Das ist ein anderer und da bin ich dann hin, weil ich bin Raucher und (...) Rauchen, so ganz problemlos ist das natürlich nicht, das ist klar. Ich würde auch lieber nicht rauchen, aber bei so viel Zeit und so weiter und er ist Raucher, da geh ich doch lieber erst mal zu $X X X$, wenn der raucht. (Q 17:32; $49 \mathrm{~J} ., \mathrm{m})$

Zudem erwarteten die befragten Patienten vom Arzt ein gewisses Ausmaß an Empathie. Sie wollten den Eindruck haben, dass der Arzt mit innen mitzufühlen vermochte, sie unterstützte, Hoffnung vermittelte und sie auf einer emotionalen Ebene ernst nahm. So konnte 
Stigmatisierung vermieden werden, und der Patient entwickelte das Gefühl, gut aufgehoben zu sein. Basis für eine solche Atmosphäre war ein Mindestmaß an freundlichem Umgang.

[...]- - Er hat auch sehr viel Mitgefühl, so empfinde ich das, ne - - also wenn ich mal ganz traurig bin oder so, sagt er dann: Ja, Sie haben auch mit ganz schön viel zu kämpfen. Und er erkennt es auch einfach an. - - Er hat mich auch mal gelobt, dass [ich] für meine Erkrankung eigentlich sehr gut mitarbeite. (Q 8:6; $38 \mathrm{~J} .$, w)

P: Da haben die soviel Empathie, ja. So grade fällt mir da ein, so in dem hohem Maße, also, - - dass er sich Zeit nimmt, um mehr mit dem Patienten und er hat mich auch damals nicht rausgeschmissen oder so was, sondern hat mich durchgetragen, bis ich dann aus dieser tiefen Depression wieder auftauchte und dann langsam wieder sprechen lernte und lesen lernte und essen lernte. [...] (Q 10:2; 62 J., m)

Fehlte jedoch dieses Mitgefühl und Einfühlungsvermögen in die Situation des Patienten, so hatte das deutlich negative Auswirkung auf die Ausbildung einer guten, tragfähigen Beziehung.

[P:] Also, wenn man jemandem sagt, also man ist grade mal frisch erkrankt an so einer Sache und man kriegt so die Prognose einem: Ja gehen Sie davon aus, dass Sie ihr Leben lang Medikamente nehmen müssen. - - Da habe ich einfach das Gefühl, da bin ich nicht gut aufgehoben, weil der will ja gar nicht mit Dir daran arbeiten, dass es vielleicht anders auch sein könnte, ne. [...] und wenn man nur die Statistiken liest, dann glaubt man eben auch nicht mehr so sehr - - - an die Möglichkeit zur Gesundung. (Q 8:90; 38 J., w)

[P:] Und da ist halt das Problem da gewesen, dass ich halt gemerkt hab, ich bin nicht mehr ernst genommen worden. Da wollte ich nicht der ldiot sein und bin halt gegangen, hab gesagt, gut, dann können wir nicht mehr zusammenarbeiten. (Q 19:5; 35 J., w)

Ein eindrückliches Negativbeispiel schilderte ein Patient, bei dem die sehr unpersönliche Art des Arztes ein ausgeprägt schlechtes Gefühl hervorgerufen hatte.

P: Ich habe Psychiater kennen gelernt, die sich sehr - - äh - - so distanziert sich zu Patienten verhalten haben, dass man sich nicht mehr als Mensch wähnte, sondern als - - irgendwie da herumsitzend - - als Objekt. Einfach, dass man halt nicht persönlich angesprochen wird, nicht begrüßt wird beim Reinkommen. Man kriegt irgendwie ein_, ein_, ein - - ja, so ein Fragebogen dann irgendwie - - äh-- sagt. Und, äh, ich hatte da mal einen Psychiater, der nebenbei dann immer in sein Diktiergerät sprach, und dann von Patient in der dritten Person halt sprach. Super unangenehm, also -- schlechter kann man es eigentlich nicht machen. (Q 9:68; $26 \mathrm{~J} ., \mathrm{m})$

Neben den Persönlichkeitsaspekten des Arztes spielte auch die Wahrnehmung über seine fachliche Kompetenz eine wichtige Rolle, um eine vertrauensvolle therapeutische Beziehung aufzubauen. Die Patienten wollten sich auf die fachliche Kompetenz des Arztes verlassen können. Diese Studie beleuchtet nicht die objektive Kompetenz, sondern die subjektive Einschätzung der ärztlichen Kompetenz durch die befragten Patienten. Für sie war es wichtig, 
dass der Arzt eine zuverlässige Diagnose stellen konnte und im Rahmen der Schizophreniebehandlung in der Lage war, Frühzeichen der Psychose zu erkennen und rechtzeitig zu intervenieren. Ebenso war es wichtig, dass der Arzt sich mit einem breiten Spektrum von therapeutischen Möglichkeiten auskannte und auch ergänzende Behandlungsangebote und Alternativen zu den schulmedizinischen Ansätzen aufzeigen konnte.

P: Naja, also, zur Ärztin gehe ich ja nur, wenn ich krank bin. Und dann schildere ich ihr meine Symptome und dann muss sie eben sagen: So, das und das haben sie jetzt. Muss sie wissen, nicht ich. (Q 5:50; $55 \mathrm{~J} ., \mathrm{w})$

[P:] nach diesem Arztbesuch bei ihm, habe ich so gedacht: Oh, das ist ja eigentlich toll. Da könnte es ja auch weitergehen. Also, wie ich mich in Griff kriege, wenn der andere bereit ist, äh -- auch da--da also zu beobachten, und daran mit einem zu arbeiten, also sich zu beobachten, ne. Weil ich muss ja immer mehr lernen, Frühzeichen zu erkennen. Also von daher war das letzte_, der letzte Termin da auch ganz gut. (Q 8:109; $38 \mathrm{~J} ., \mathrm{w})$

Die Einschätzung der Kompetenz war ein Kriterium, das bei der Auswahl des Arztes einfloss, obwohl fachliche Kompetenz alleine nicht ausreichte, jemanden als guten Arzt zu empfinden. Als Maßstab für die Kompetenz setzten die Patienten einerseits den Behandlungserfolg an und andererseits schätzen sie den Arzt kompetenter ein, wenn er sich gemäß ihrer Erwartungen verhielt.

I: Woran merken sie das, dass er diagnostisch eine hohe Kompetenz besitzt? $P:$ Äh, ja, ich selber habe eine langjährige psychiatrische Erfahrung. Daneben eine langjährige psychotherapeutische Erfahrung, psychologische und tiefenpsychologische Erfahrung, und äh, habe auch Literatur studiert. Also sowohl in theoretischer als auch in praktischer Hinsicht, äh, -- ja äh, könnte man sagen, dass ich ein bisschen Zugang zur Psychologie und Psychopathologie gefunden habe. Und ich habe meine eigene Diagnose gestellt und meine eigene Erfahrung, und der Hausarzt hat die gleiche Diagnose gestellt wie ich, --ja. (Q 1:89; 52 J., m)

I: Und Sie haben gesagt, der Dr. XXX, der behandelt Sie anständig! Woran merkt man das genau?

P: [...] Da glaub ich jetzt immer dran, dass er mir richtig alles verschreibt wie es sich gehört. (Q 15:5; 79 J., m)

Als negativ beschrieben die Patienten ihren Eindruck, wenn Hausärzte eher ungenügende Kenntnis und Erfahrung in der Psychopathologie hatten.

P: Also viele Hausärzte haben mit psychischen Problemen halt nicht so die Ahnung, die sind dann sehr neugierig und fangen da an, die Leute auszufragen, und das ist halt manchmal nicht so angenehm, wenn es einem sowieso schon irgendwie schlecht geht. - - Wenn man so dieses Interesse, Neugier und das bringt nichts, weil man da teilweise nichts drüber erzählen will, [...] weil man sich in einer Stresssituation wähnt und dann noch ausgefragt wird, -- das bringt nichts. (Q 9:67; $26 \mathrm{~J} ., \mathrm{m})$ 
[P:] Da hat sie reingeschrieben Psychose und paranoid oder so -- paranoider Typ, sogar - - auch wieder so ein bisschen gegen meinen Strich. Ja - - und da habe ich das Gefühl gehabt, der kennt das nicht und will sich auch nicht damit befassen. (Q 14:49; $36 \mathrm{~J} ., \mathrm{m})$

\subsubsection{Autonomie}

Als ein wichtiges Merkmal einer guten Arzt-Patient-Beziehung wurde immer wieder die Autonomie des Patienten genannt. Die Patienten beanspruchten für sich, dass ihre Meinung ernst genommen und respektiert wird. Sie wollten in der Gestaltung der Therapie und ihrer Arbeitssituation selbst entscheiden und übernahmen eigene Verantwortung für ihre Entscheidungen und ihr Handeln.

[P:] Und dass eigentlich so ein gutes Verhältnis da ist, dass immer ich mich etwa gleichwertig fühle. Das ist auch wichtig, dass nicht nur er derjenige ist, der über mich bestimmt oder seine Ansichten durchsetzt, sondern dass er mir halt auch die Autonomie zugesteht, auch selber zu handeln. Und dabei eben nicht ins andere Extrem übergeht, nach dem Motto „es ist mir egal, was sie machen", sondern er sich auch die Mühe macht und die Geduld hat, eben abweichende Faktoren oder Dinge zu thematisieren. (Q 4:44; $30 \mathrm{~J} ., \mathrm{m})$

Als negativ erlebt wurde es hingegen, wenn der Arzt die Eigenständigkeit des Patienten nicht anerkannte, sehr autoritär auftrat, im schlimmsten Falle sogar mit Sanktionen drohte, wenn der Patient sich nicht an seine Anordnungen hielt.

[P:] Hatte ich schon doch mal die Erfahrung gemacht, dass der Hausarzt dann versucht hat, zu drohen mit Einweisung und Psychiater benachrichtigen und so was, und das ist eigentlich sehr unschön, - - also, das kann man sich auch sparen, denke ich. - (Q 9:66; $26 \mathrm{J.}, \mathrm{m})$

\subsubsection{Arzt-Patient-Passung und Kommunikation}

Unter dem Begriff der Arzt-Patient-Passung wurden die „Chemie“ oder „Wellenlänge“ zwischen zwei Personen beschrieben, also Persönlichkeits- oder Charakterzüge, die zur Ausbildung einer guten Arzt-Patient-Beziehung beitrugen, jedoch nur schwer objektiv zu fassen waren. Darunter fallen zum Beispiel auch spontane Sympathien oder Antipathien und eine gut funktionierende, reibungslose Kommunikation.

[P:] Und so machen das ja auch dann, glaube ich, die meisten Menschen, dass man, wenn das eben_, wenn man spürt, dass es halt nicht so da, der Draht, dass man dann vielleicht eben woanders hingeht, ne. (Q 8:31; $38 \mathrm{~J} ., \mathrm{w})$

[P:] Aber Frau F. hat das so gesagt, die Chemie muss stimmen, und da, das denk ich auch, das ist schon wichtig, und das sind so Sachen, z. B. wo ich mir auch eine Freundin oder ein Freund aussuchen müsste. Ich würde, das sind so Sachen, die kann man 
schlecht beschreiben, die laufen oft so auf zwischenmenschlicher Ebene. Da kann ich nicht sagen, der und der müsste so und so aussehen, sondern das ist immer auch so ein bisschen Glück, wenn man zu jemanden hinfindet, mit dem man sich gut unterhalten kann und mit dem man auch offen sein kann. (Q 18:23; 54 J., w)

Wenn diese "Chemie" allerdings nicht stimmte, so kam es bei einigen Patienten vor, dass der Kontakt zum Arzt nicht nur nicht hilfreich war, sondern sich sogar negativ auf das Wohlbefinden des Patienten auswirkte.

P: Ja, es kommt dann auch eben wieder auf die Person an. Wenn ich jetzt vielleicht mit der - - ist das so ein Psychiater, mit dem ich oft rede, mit dem ich vielleicht nicht so zurecht komme, ist das noch beklemmender sogar, ne - - (Q 6:70; $36 \mathrm{~J} ., \mathrm{m})$

[P:] Ich war kurz vorher bei einer Fachärztin hier in der Stadt und die hat eigentlich einen sehr guten Ruf. Und bei der hatte ich aber das Gefühl, -- die konnte sich gar nicht für mich sensibilisieren, und da war ich hinterher also auch ein bisschen traurig, ne. - äh - und da habe ich dann jetzt gedacht, das liegt vielleicht daran, dass sie vielleicht völlig überfüllt ist oder dass der Draht nicht gestimmt hat. (Q 8:55; $38 \mathrm{J.}$., w)

Auch Übereinstimmungen im Welt- und Menschenbild waren Elemente, die sich positiv auf die Beziehungsbildung auswirkten.

[P:] Im Gegenteil, ich bin für Naturwissenschaft. Meine aber, dass auch die geistige Seite der Medaille berücksichtigt werden muss. Da versuche ich meinen Psychiater so in den letzten Jahren bisschen aufgeschlossener für zu machen. Durch Gespräche und durch Literatur, und er scheint doch auch aufgeschlossener zu sein, etwas. Ja, und das finde ich sehr positiv. (Q 1:64; $52 \mathrm{~J} ., \mathrm{m})$

[P:] Man kann sicherlich bei xyz-Arzt - - auch so was finden, aber bei mir ist eben so - also, die Anthroposophie, die beschäftigt sich ja auch ein bisschen anders noch mit, [...] Also vom Menschenbild und von der - - von den Sachen, die Menschen durchmachen. [...] Auf jeden Fall ist es bei mir so, weil ich auch nach solchen Dingen immer suche -also - - äh - - Das mag jetzt ein Zufall sein, dass er sich damit beschäftigt. (Q 8:10; $38 \mathrm{~J} ., \mathrm{w})$

Die Übereinstimmung im Menschen- und Weltbild machte sich auch unter therapeutischen Aspekten bemerkbar, da sie direkt mit den Einstellungen zur Schulmedizin und therapeutischen Alternativen dazu in Zusammenhang gebracht werden konnte. Ähnlichkeiten auf diesem Gebiet wurden von den Patienten als positiv erlebt, Diskrepanzen hingegen als negativ.

\section{I: Und vieles ist Ihnen in den Gesprächen mit Herrn XXX klargeworden?}

$P$ : Ja, Ja, da hab ich gelernt, dass meistens also, fast alles kann man sagen, so zu deuten, wie das psychoanalytisch eben grade möglich ist! Ich meine auch schon, dass das zutreffend ist, wir sind schon Anhänger der Psychoanalyse, der klassischen. (Q 10:12; 62 J., $\mathrm{m}$ ) 
[P:] Nur halt wie ich am Anfang schon ansprach, dass der Psychologe bisschen andere Ansichten manchmal hat wie ich von Psychotherapie, und ich wünsch mir halt jemand, bei dem ich noch ein bisschen besser mich vielleicht aufgehoben fühle. (Q 4:8; $30 \mathrm{~J} ., \mathrm{m})$

Die gleiche „Wellenlänge“ und ideologische Übereinstimmungen machten jedoch allein noch keine gute Beziehung aus. Der Arzt musste auch in der Lage sein, angemessen mit dem Patienten zu kommunizieren. Viele der befragten Patienten wünschten von ihrem Arzt Informationen über die Erkrankung und Therapie (einschließlich der Medikamente), da er oftmals die primäre Informationsquelle auf diesem Gebiet war. Außerdem sollte er in der Lage sein, sein Vorgehen und seine therapeutischen Vorschläge in einer dem Patienten verständlichen Weise darzulegen.

I: Also, Informationen fehlen Ihnen da eigentlich auch?!

$P$ : Ja. Also mittlerweile geht's, weil ich dann auch selber nachgefragt hab und so, aber - - ich hätte mir schon ein paar mehr Erklärungen gewünscht! (Q 11:5; 23 J., w)

P: Also ich finde, sie sollten da auch schon immer informieren, weil sie auch wissen, so - - und den nächsten Schritt vorhaben, wie es so weitergeht, welche Perspektiven es da gibt. Ob da vielleicht auch ein anderer Arzt wieder in Frage kommt, eine neue Therapieform oder vielleicht so eine Art Behandlungsplan einfach. [...] (Q 6:73; $36 \mathrm{J.}, \mathrm{m}$ )

Zusätzlich zu den Inhalten der Information ist es für eine gute Beziehung aber auch wichtig, wie sie kommuniziert werden. Zum einen sollte es in einer verständnisvollen Art geschehen und zum anderen in einem Ausmaß, das dem Patienten angemessen ist. Die Patienten erwarteten jedoch nicht nur eine funktionierende Kommunikation innerhalb der Beziehung, sondern sahen den Hausarzt auch als Schnittstelle zu den anderen behandelden Personen (vgl. auch Abschnitt 5.2.1, inbesondere Q 19:16).

[P:] Dieser Arzt sagte dann zu mir: Gehen Sie davon aus, Sie müssen Ihr Leben lang Medikamente nehmen. Und die Krankheit war frisch ausgebrochen und die Prognose äh - - also, da habe ich mich so geärgert drüber, weil ich gesagt habe, - - - also es war ja gar nicht klar, ob die Krankheit - - (...) Zeiten wiederkommt und so. Und damals hat mich dieser Ausspruch, obwohl er ja im nachhinein Recht hat damit, war der Ausspruch mir aber zu hart von ihm. Und dann dachte ich äh -- also, da habe ich mich natürlich überhaupt nicht äh - - aufgehoben gefühlt dann. (Q 8:81; 38 J., w)

[P:] Weil viele Hausärzte sagen, ja wenn ich meinen Patienten die ganzen Nebenwirkungen nun noch erkläre, dann sitzt er drei Tage später bei mir in der Praxis und hat von den Nebenwirkungen fünfzehn. Und ich denke, wenn man da-- offen irgendwie dran herangehen würde, dann wäre das auch nicht der Fall. (Q 9:61; 26 J., m)

\subsubsection{Soziales Umfeld}

Auch das soziale Umfeld hatte Auswirkungen auf die Arzt-Patient-Beziehung. So empfanden es einige Patienten zum Beispiel als Barriere, wenn ihr Arzt aus einer anderen sozialen Schicht kam und beide diese Differenz nicht zu überbrücken vermochten. Andererseits 
konnte es verbindend wirken, wenn man auf diesem Gebiet Gemeinsamkeiten entdeckte, wie zum Beispiel die Herkunft aus dem gleichen Ort oder Stadtviertel.

[P:] Und - - - ja - - da man als Arzt aus einer anderen Gesellschaftsschicht kommt, ist das einem manchmal so ein bisschen fremd, wie es den etwas ärmeren Leuten irgendwie geht. Das ist heute noch so ein Problem, aber hat sich auch schon gebessert. Auch wenn er mir manchmal etwas weltfremd vorkommt, der gute Mann. [...] (Q 9:54; 26 J., m)

[l:] Würden Sie sagen, das sind bestimmte Eigenschaften vielleicht, wo Sie sagen, das macht es freundschaftlich, das macht den Umgang gut?

$P$ : Na ja, ich wohne auch im vorderen Westen. [...] ich denke das hat so ein bestimmtes Flair. Ich wohn da jetzt selbst, wo ich wohne seit 15 Jahren. (Q 17:46; 49 J., m)

Ebenso hatte das soziale Umfeld Einfluss auf die Auswahl des Arztes, indem zum Beispiel innerhalb der Familie oder im Freundes- und Bekanntenkreis Empfehlungen für oder gegen einen bestimmten Arzt ausgesprochen wurden. Es bestand dann schon vor dem eigentlichen Kennenlernen ein Bild vom Arzt, das sich positiv oder negativ auf die Beziehungsbildung auswirken konnte.

[P:] Also da kommt ja noch auf den Weg, dass man von Freunden Empfehlungen hat auch noch, dass die sagen, ja, der soll gut sein, da kann man hingehen. (Q 11:46; 23 J., w)

Neben den Auswirkungen, die das soziale Umfeld auf der persönlichen Ebene der ArztPatient-Beziehung hatte, wurden auch immer wieder dessen Bedeutung für die Therapie beschrieben. Zum einen erlebten es die Patienten als gut, wenn der Arzt sie als Teil ihres sozialen und familiären Umfelds sah und diese Aspekte auch in die Behandlung einfließen ließ. Dies geschah insbesondere dann, wenn der Hausarzt in der Funktion des Familienarztes auch Angehörige des Patienten betreute. Im Rahmen der Therapie konnte er so auch vermittelnd zwischen Umfeld und Patienten tätig werden.

[I:] Und ähm, das ist spannend, dass es so ein richtiger Familienarzt ist. [...] Könnten Sie da mir noch mehr drüber sagen? Gefällt Ihnen das?

P: Mir gefällt das sehr gut. Weil man, weil die einen ja richtig kennen schon von Anfang an und sie haben bis jetzt immer geholfen, wenn man etwas hatte. Es hat immer einen Sinn gehabt. Deshalb sind wir, unsere ganze Familie ist mit dem Arzt einverstanden. [...] (Q 5:39; $55 \mathrm{~J} ., \mathrm{w})$

P: Na ja, der Hausarzt sollte halt auch darum bemüht sein, das Leben für den Kranken halt auch so angenehm wie möglich zu machen und auch für die Umwelt halt, dass dann ein Gleichgewicht hergestellt wird, weil viele psychisch Kranke sind oft auch eine Last für ihre Umwelt, so will ich mal sagen. Da muss eben die Balance auch gefunden werden. (Q 23:21; 41 J., m)

Im Gegensatz dazu gab es aber auch Patienten, die bewusst eine Trennung zwischen ihrem sozialen Umfeld und ihrer ärztlichen Behandlung herbeiführten. 
[P:] Also ich selber hab auch den Hausarzt gewechselt, weil ich nicht mehr bei demselben Hausarzt sein wollte wie meine Eltern. (...) (Q 11:37; 23 J., w) 


\section{Diskussion}

In der vorliegenden Arbeit wurde untersucht, welche Rollen Hausarzt und Facharzt aus Sicht der Patienten in der ambulanten Schizophreniebehandlung einnehmen. Merkmale der hausärztlichen Behandlung schizophrener Patienten (wahrgenommene Stärken und Schwächen) wurden in den Kontext der ambulanten Behandlungsangebote gestellt. Besonderes Augenmerk galt den Faktoren, die zur Ausbildung einer guten Arzt-Patient-Beziehung und Förderung von Medikamentencompliance schizophrener Patienten beitragen. Im Folgenden werden die wesentlichen Ergebnisse der Untersuchung diskutiert und zu aktuellen Forschungsergebnissen auf diesem Gebiet in Beziehung gesetzt. Jeweils am Ende dieser einzelnen Punkte werden Schlussfolgerungen für eine Optimierung der Schizophreniebehandlung in der Allgemeinarztpraxis abgeleitet, die insbesondere die Verbesserungsvorschläge der $\mathrm{Pa}$ tienten einbeziehen. Abschließend wird die Methodik der Arbeit diskutiert.

\subsection{Die Rolle des Hausarztes in der ambulanten Schizophreniebehandlung}

Bereits während der frühen Phase der Datenerhebung zeigte sich, dass in dieser Untersuchung eine Versorgungssituation vorlag, die deutlich von der durch HAASEN und STARK (1997) skizzierten abwich. So wurde weit weniger als ein Drittel der Patienten auch im Rahmen der schizophrenen Erkrankung ausschließlich vom Hausarzt betreut. Zumeist fand eine Doppelbetreuung statt, in der die Patienten dem jeweiligen Arzt recht eindeutige Zuständigkeitsbereiche zuordnen konnten (vgl. Abschnitt 5.2 und 5.3. Aus diesen Zuständigkeitsbereichen lässt sich die Rolle skizzieren, die der Hausarzt aus Sicht der schizophrenen Patienten einnahm oder einnehmen sollte, was bisher kaum untersucht wurde.

\section{Der Hausarzt als Körperarzt}

Für viele Patienten war der Hausarzt in erster Linie „Körperarzt“. Sie gingen mit somatischen Beschwerden zu ihm und erwarteten eine suffiziente Behandlung und die Lösung des Problems. Der Hausarzt wurde von den von uns befragten Patienten im Vergleich zum Facharzt meist unregelmäßiger und mit konkretem Anlass konsultiert. Hier deckte sich das Aufgabenfeld des Hausarztes weitestgehend mit den Vorstellungen und Ansprüchen an die hausärztliche Betreuung, die auch nicht psychisch kranke Patienten in anderen Befragungen (KLINGENBERG et al. 1996, GROL et al. 1999) hatten (vgl. auch Abschnitt 2.2.3). Die 
Differenzierung zwischen „Körperarzt“ und „Seelenarzt“ betonten und schätzten Patienten insbesondere dann, wenn ein funktionierender interdisziplinärer Austausch bestand. Da die mittlere Krankheitsdauer der befragten Patienten über 20 Jahre betrug (vgl. Abschnitt 5.1 , konnten sie während dieser langen Erkrankungsdauer vermutlich bereits ein funktionierendes Netz ambulanter psychiatrischer Betreuung etablieren.

Als „Körperarzt“ ist dem Hausarzt schon deshalb eine wichtige Bedeutung beizumessen, weil schizophrene Patienten eine höhere Morbidität und Mortalität auch für somatische Erkrankungen aufweisen als Personen ohne eine schwere psychische Störung (BROWN 1997). Diese Patienten würden also auch in Bezug auf somatische Erkrankungen eine erhöhte Aufmerksamkeit der Hausärzte erfordern, was aber gemäß einer aktuellen englischen Studie nicht der Versorgungsrealität entspricht (ROBERTS et al. 2006). Trotz des erhöhten Risikos wurden bei schizophrenen Patienten grundlegende Gesundheits-Checks (z. B. Kontrolle des Blutdrucks oder Serumcholesterins) mit geringerer Wahrscheinlichkeit durchgeführt als bei Patienten einer Kontrollgruppe mit Asthma und einer allgemeinen Kontrollgruppe. Die Situation in Deutschland dürfte ähnlich sein. Wahrscheinlich würde ein gesteigertes Bewusstsein für die besondere Situation schizophrener Patienten im Bezug auf somatische Erkrankungen ausreichen, damit Hausärzte der ihnen zugeordneten Aufgabe als „Körperarzt“ ohne erheblichen Mehraufwand besser gerecht werden könnten.

\section{Erkrankungsbeginn und Früherkennung}

Anders hingegen sahen die Erwartungen der Patienten bei Erkrankungsbeginn aus. Hier kam dem Hausarzt auch im Bezug auf die psychischen Erkrankung eine besondere Rolle zu, da er den meisten Patienten bereits vorher bekannt gewesen war und dadurch einen „Vertrauenskredit“ besaß. Oftmals war der Hausarzt der erste Arzt, der vom Patienten oder seinen Angehörigen Hinweise auf die Symptome erhielt. Die befragten Patienten wünschten sich einen Hausarzt, der die psychische Störung erkennen und den Betroffenen in die fachärztliche Betreuung weiterleiten kann. Hier scheint eine Diskrepanz zwischen den geäußerten Erwartungen und der Versorgungsrealität zu bestehen. Das groß angelegte Swiss Early Psychosis Project (SWEPP) befragte 1089 Hausärzte in der Schweiz (Rücklaufrate 28\%) über die Behandlung von Schizophrenie in ihrer Praxis (ANDOR et al. 2005). Die Autoren konnten zeigen, dass wichtige Frühzeichen einer Psychose nur von 33\% der teilnehmenden praktischen Ärzte genannt wurden, was Defizite im Bereich der Diagnostik vermuten lässt. Wenn man jedoch von einem Lebenszeitrisiko einer Schizophrenierkrankung von $1 \%$ der Bevölkerung und einer entsprechend geringeren Zahl an jährlichen Neuerkrankungen ausgeht, dürfte der einzelne Hausarzt nur äußerst selten eine Erstmanifestation von Schizophrenie in seiner Praxis sehen. 


\section{Der Hausarzt als psychiatrischer (Mit-)Behandler}

Neben seiner Funktion als „Körperarzt“ hatte der Hausarzt auch eine Funktion bei der Behandlung der psychischen Erkrankung, es entstand ein Überschneidungsbereich mit der fachärztlichen Behandlung. Hier beschrieben die interviewten Patienten, dass der Hausarzt in der Erhaltungsphase oftmals die vom Facharzt verordnete Medikamententherapie fortführte sowie die somatischen Parameter (z. B. Blutwerte) und mögliche unerwünschte Wirkungen überwachte. Im Rahmen des bereits o. g. SWEPP konnten ANDOR et al. (2005) bezüglich der Einschätzung zur Notwendigkeit, Art und Ausmaß einer Erhaltungstherapie zur Rückfallsprophylaxe zeigen, dass nur etwa die Hälfte der Ärzte eine Therapieempfehlung gemäß internationaler Richtlinien gab. Besser schnitten hierbei diejenigen Hausärzte $a b$, die ein psychiatrisches Weiterbildungsjahr absolviert oder in kürzerer Vergangenheit eine themenbezogene Fortbildung besucht hatten. Hier wurde also ein Fortbildungsbedarf aufgezeigt, der auch von den befragten Patienten wahrgenommen wird, die mehrfach den Wunsch nach besserer Kenntnis des Hausarzts im Bereich der Schizophrenie äußerten.

\section{Der Hausarzt als Ansprechpartner und Familienarzt}

Im Bezug auf ihre psychiatrische Erkrankung wünschten sich einige der befragten Patienten von ihrem Hausarzt zudem Gesprächsbereitschaft und Aufgeschlossenheit für ihre psychische Situation (Patienten 4,6,8). Hier überschnitten sich medizinische und soziale Betreuung, insbesondere dann, wenn der Hausarzt als Familienarzt auch Kontakt zu den Angehörigen der Patienten hatte oder von den Patienten als Ansprechpartner für alltägliche Probleme zu Rate gezogen wurde. Der Hausarzt war zudem eine wichtige Informationsquelle für die Patienten und oft auch ihre Angehörigen. Er informierte nicht nur über die Erkrankung selbst, sondern auch über die Therapie (Medikamente und begleitende therapeutische Ansätze). Da PIETSCHEL-WALZ et al. (2006) zeigen konnten, dass durch Psychoedukation von Patienten und nahen Angehörigen, respektive Bezugspersonen, die Compliance erhöht und die Rehospitalisationsrate gesenkt werden kann, kann der Hausarzt hier eine wichtige Funktion übernehmen.

\section{Der Hausarzt als Gatekeeper}

Soweit Hausärzte die o. g. Aufgaben tatsächlich übernahmen, erfüllten sie für die Patienten oft eine Gatekeeper-Funktion im Zentrum der Gesundheitsversorgung. Beim Hausarzt „liefen die Fäden zusammen“, er übernahm eine koordinierende Rolle (vgl. Abbildung 6.1). Hierzu benötigte er den Überblick über die Behandlung des Patienten und entsprechendes Fachwissen, um zum richtigen Zeitpunkt an einen Facharzt zu überweisen und die fachärztlich eingeleitete Behandlung korrekt fortzuführen. In diesem Bereich wurden von den Patienten der vorliegenden Untersuchung Defizite beim Hausarzt wahrgenommen, die in Abschnitt 6.2 eingehender diskutiert werden. Schwächen sahen Patienten ebenso in der Kommunikation 


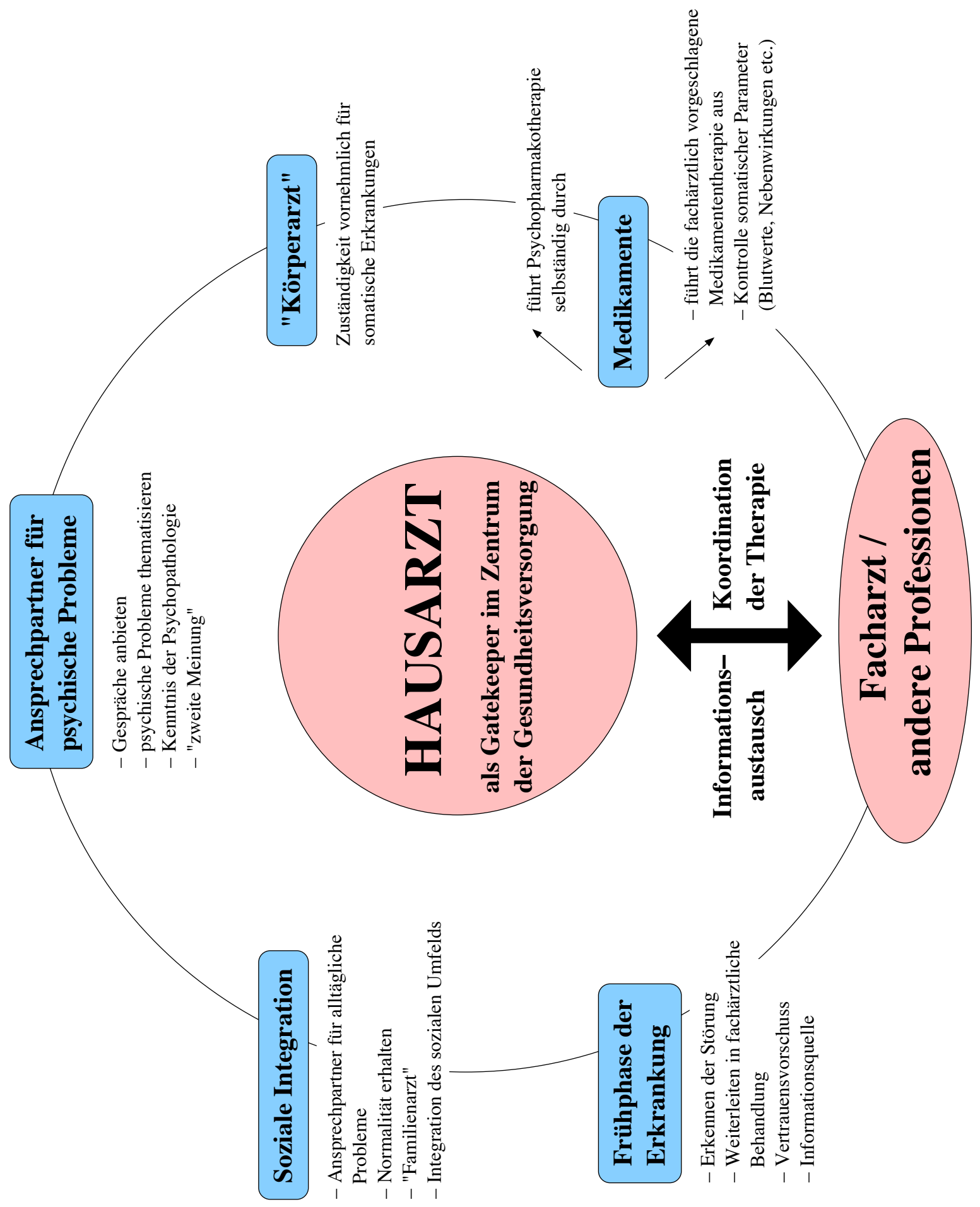

Abbildung 6.1: Die Rolle des Hausarztes in der ambulanten Schizophreniebehandlung 
zwischen den behandeInden Ärzten. In vorausgegangenen Untersuchungen äußerten auch Hausärzte aus ihrer Sicht Verbesserungsbedarf in der interdisziplinären Kommunikation und Koordination (FALLOON et al. 1996, SPIESSL und CORDING 1999, DE CRUPPE et al. 2006).

Dem gegenüber steht eine aktuelle deutsche Untersuchung, in der der wahrgenommene Nutzen einer Überweisung aus Sicht von Hausarzt, Facharzt und Patient prospektiv untersucht wurde (ROSEMANN et al. 2006). Hier konnte bei Überweisungen zu Fachärzten somatischer Disziplinen gezeigt werden, dass alle drei beteiligten Parteien in den meisten Fällen mit dem Resultat der Überweisung zufrieden waren (83\% der Patienten, $77 \%$ der Hausärzte) respektive sie für angemessen hielten (91\% der Fachärzte). Unerwartet war hier vor allem, dass die Zufriedenheit der Patienten größer war, wenn die Überweisung vom Hausarzt (nicht von ihnen selbst) initiiert wurde. Die Gründe hierfür wurden nicht weiter untersucht. Es wäre sicher interessant zu erfahren, welchen Einfluss etwa eine gute Arzt-Patient-Beziehung, in der der Patient den fachlichen Kompentenzen seines Hausarztes vertraut, in diesem Punkt hat. Auch fehlen Untersuchungen, die diese Fragestellung nach dem subjektiven Nutzen einer Überweisung zum psychiatrischen Facharzt gezielt für ein Kollektiv schizophrener Patienten in hausärztlicher Behandlung bearbeiten.

Trotz einiger Überschneidungsbereiche, die von Patient zu Patient individuell unterschiedlich gewünscht und zugeordnet wurden (Medikamententherapie, Gespräche) grenzte sich die Rolle des Hausarztes klar von der des Facharztes ab, den die Patienten dieser Untersuchung fast immer exklusiv in der Betreuung der psychischen Erkrankung sahen (,Seelenarzt").

\subsection{Ressourcen und Defizite von Hausarzt und Facharzt}

Aus den Aufgaben und Erlebnissen, wie sie die Patienten wünschten bzw. erzählten ergeben sich die besonderen Stärken und Schwächen der hausärztlichen Versorgung. Der in den Interviews wesentlich hervorgehobene Vorzug der Behandlung durch den Hausarzt ist, dass ein gewisses Maß an Normalität erhalten bleibt („man sich als normaler Mensch fühlt“), was den Aufbau oder die Aufrechterhaltung der Arzt-Patient-Beziehung erleichtern kann. Einige Patienten hatten seltener beim Hausarzt als beim Facharzt die Entwicklung eines „Tunnelblicks" erlebt, bei dem nur noch die Psychopathologie des Patienten und nicht mehr das Normale des einzelnen Menschen gesehen wurde. So fühlten sich einige Patienten gegenüber dem Hausarzt „normaler“ und weniger krank, was sie positiv werteten. Sehr eindrücklich schilderte eine Patientin, dass sie die Notwendigkeit der fachärztlichen Behandlung als Indikator für die Schwere ihrer Erkrankung erlebte. Nachdem der Hausarzt sie lange Zeit auch im Bezug auf die Schizophrenie ausschließlich selbst behandelt hatte, musste er beim Auftreten von Schwierigkeiten schließlich einen Psychiater hinzuziehen (Patientin 8). Bereits aus diesem Grund war sie der Behandlung durch einen Facharzt gegenüber eher negativ eingestellt; die Schwelle, den Hausarzt aufzusuchen, schien dagegen geringer zu sein. Mit 
diesen Erfahrungen korrespondiert, dass psychiatrische Erkrankungen und insbesondere die Schizophrenie mit erheblichem Stigma belegt sind (WHO 2001) und die Angst vor Stigmatisierung mit einer suffizienten Betreuung interferieren kann (CORRIGAN 2004). Gemäß SARTORIUS (2002) wird Stigma häufig auch iatrogen begünstigt. Er empfiehlt daher Ärzten, vorsichtig mit der medizinische Diagnose umzugehen. Sie sei nützlich in der Kommunikation mit anderen Fachkollegen, jedoch oft schädlich, wenn sie an Laien weitergegeben werde, die die fachliche Definition des Begriffs nicht oder nicht korrekt kennen. So legten BAG et al. (2005) zufällig ausgewählten Personen Fallvignetten vor und konnten zeigen, dass die beschriebenen Personen insbesondere dann als gefährlich und unberechenbar eingeschätzt wurden, wenn die Probanden eine psychotische Erkrankung zu erkennen glaubten. Sie gaben zudem an, eine erhöhte soziale Distanz zu diesen Patienten einhalten zu wollen. Angst vor Stigmatisierung könnte also dazu beitragen, dass die Schwelle, ärztliche Behandlung in Anspruch zu nehmen, beim Hausarzt geringer ist als beim Facharzt. Diese Scheu vor der Stigmatisierung zeigte sich möglicherweise schon bei der Antwort auf die Frage nach der eigenen Krankheit (vgl. Tabelle 5.1): Hier verwenden nur acht der 20 Patienten den Begriff „Schizophrenie“, während er ansonsten gemieden und umschrieben wird.

Als positiv erlebten es viele Patienten, bereits eine lange Beziehung zum Hausarzt zu haben. Es bestand eine Vertrauensbasis, die insbesondere beim Ausbruch der psychischen Erkrankung eine wesentlich tragende Rolle für den Patienten spielen konnte. Diese Kontinuität der Betreuung durch einen Arzt ging außerdem mit einer signifikant höheren Zufriedenheit der Patienten bezüglich des Arztbesuchs einher, wie eine Befragung von 3.918 norwegischen Patienten in Allgemeinarztpraxen zeigen konnte (HJORTDAHL Und LFRUM 1992).

Weiterhin erlebten es mehrere Patienten als vorteilhaft, wenn ihr Arzt einen Einblick in ihre private und soziale Situation hatte. Dies war zum einen so, wenn der Arzt schon lange bekannt war, zum anderen aber auch, wenn er im Sinne eines Familienarztes mehrere Mitglieder der Familie betreute. Der Einblick in das persönliche Umfeld des Patienten ermöglichte eine individuelle, mehrdimensionale Behandlung (z. B. unter Einbeziehung Angehöriger und des sozialen Umfelds) und eine tiefergehende Beziehung. Dies wurde noch unterstützt, wenn der Hausarzt für die Patienten die zentrale Figur der Gesundheitsversorgung darstellte, wenn bei inm alle Informationen zusammenflossen und er eine koordinierende Rolle übernahm. Diese Ergebnisse entsprechen den durch die EUROPEP-Studie (KLINGENBERG et al. 1996) erhobenen Anforderungen an den Hausarzt. Auch hier galt als besonders wichtig, dass Hausärzte schnell verfügbar sind, Zeit zum Zuhören, Reden und Erklären haben und krankheitsrelevante Information zur Verfügung stellen.

Die Ergebnisse dieser Untersuchung verweisen jedoch auch auf typische Defizite der hausärztlichen Betreuung. Hierzu zählt vor allem die von manchen Patienten als ungenügend wahrgenommene fachliche Kompetenz auf dem Gebiet der Psychiatrie und Psychopathologie. Dies war oftmals der Grund, weshalb sie die Behandlung der Psychose durch ihren Hausarzt ablehnten oder aber eine bessere Ausbildung der Mediziner auf diesem Gebiet forderten. Diese offenbar nicht ausreichende Kenntnis konnte dann sogar dazu führen, dass 
ein Patient das Gefühl hatte, der Hausarzt begegne ihm mit Vorurteilen (Patient 9). Weiterhin wurde bemängelt, dass Hausärzte viel zu wenig Zeit hätten, um psychische Erkrankungen zu behandeln; aufgrund der hohen Anzahl der zu betreuenden Patienten sei die Zeit für den einzelnen zu kurz. Diese Einschätzung deckt sich recht gut mit den Ergebnissen einer Befragung von FALLOON et al. (1996), in der $80 \%$ der Hausärzte meinten, zu wenig Zeit für die Betreuung psychisch Kranker zu haben; $90 \%$ wünschten sich eine fachliche Unterstützung durch entsprechend weitergebildete Kollegen. Die Ergebnisse beider Studien unterstreichen somit noch einmal den Handlungsbedarf auf diesem Gebiet. Es stellt sich allerdings die Frage, zu welchem Zeitpunkt eine entsprechende Intervention stattfinden sollte. Zum einen könnte der psychiatrischen Ausbildung im Rahmen des Medizinstudiums eine höhere Bedeutung beigemessen werden, was aufgrund der hohen Inzidenz psychischer Erkrankungen durchaus gerechtfertigt erscheint. Zum anderen könnte man einen gewissen Umfang psychiatrischer Ausbildung obligatorisch in die Weiterbildung zum Facharzt für Allgemeinmedizin integrieren oder zur Voraussetzung für hausärztliche Tätigkeit machen - insbesondere vor dem Hintergrund der hohen Prävalenz psychiatrischer Störungen in Allgemeinarztpraxen (ORLEANS et al. 1985, PHILBRICK et al. 1996, OLFSEN et al. 2002).

Aus den geschilderten Erfahrungen in den Interviews lassen sich auch die Ressourcen und Defizite der fachärztlichen Betreuung herausstellen. So wurde vor allem positiv angesehen, dass Fachärzte mehr Zeit für Gespräche haben, welche in der Betreuung von Psychose-Kranken als äußerst wichtig angesehen wurden. Diese längere Gesprächszeit wurde als Grundlage genannt, eine persönliche Beziehung eingehen zu können und sich zu öffnen. Hieraus ergab sich, dass beim Psychiater auch bereits nach kürzerer Zeit eine tiefere Beziehungsebene erreicht werden konnte, als dies beim Hausarzt möglich war. Außerdem wurde als eine weitere Stärke des Facharztes seine spezifische Kompetenz im Bereich der psychischen Erkrankungen eingeschätzt. Die Patienten erhofften sich davon einen besseren Behandlungserfolg. Jedoch schien die Schwelle, sich in fachärztliche Behandlung zu begeben, gegenüber der Behandlung beim Hausarzt erhöht zu sein. Ebenso wünschten die Patienten - in Übereinstimmung mit Arbeiten von BINDMAN et al. (1997) und SPIESSL und CORDING (2000) - eine bessere Kooperation und Koordination zwischen diesen Arztgruppen.

\subsection{Begünstigende Faktoren für eine tragfähige therapeutische Beziehung aus Sicht der Patienten}

Da einige Studien zeigen konnten, dass die Arzt-Patient-Beziehung erheblichen Einfluss auf die Compliance hat, galt in dieser Untersuchung den Gütekriterien einer solchen Beziehung aus Sicht schizophrener Patienten besondere Aufmerksamkeit. Eine weitere Fragestellung beschäftigte sich damit, wie eine gute Beziehung erreicht werden kann. FRANK und GUNDERSON (1990) konnten zeigen, dass lediglich bei einer Minderheit schizophrener Patienten 
die Beziehungen zu ihrem Arzt gut sind. Die Autoren hatten jedoch nicht die Faktoren, die für die Güte bezeichnend waren, herausgearbeitet. Eine aktuelle Studie befasste sich mit der Ausbildung der therapeutischen Beziehung von Schizophreniepatienten zu ihren Psychotherapeuten zu Behandlungsbeginn (COUTURE et al. 2006). Die Autoren untersuchten den möglichen Zusammenhang zwischen Patientencharakteristka, gemessen mit der Positive and Negative Syndrome Scale (PANSS) nach KAY et al. (1987) und der Social Functioning Scale (SFS) nach BIRCHWOOD et al. (1990), und der Beurteilung der Beziehung, gemessen mit dem Working Alliance Inventory (HORVATH und GREENSBERG 1989). Eine wichtige Erkenntnis der Untersuchung war, dass es keine signifikanten Übereinstimmungen in der Bewertung der Beziehung durch die jeweiligen Therapeuten und Patienten gab. Diese offensichtlichen Diskrepanzen in der Wahrnehmung nahmen die Autoren zum Anlass, die Notwendigkeit der Erfassung der Patientenperspektive hervorzuheben (,greater attention needs to be placed on the clients' perceptions of therapist characteristics"). Hier bietet die vorliegende Arbeit Ansätze.

Die aus den Interviews erarbeiteten Gütekriterien der Beziehung konnten in drei übergeordnete Kategorien eingeteilt werden (vgl. Abschnitt 5.5): strukturelle Merkmale (z. B. Dauer und Kontakthäufigkeit der Beziehung, Infrastruktur), Passung zwischen Arzt und Patient (z. B. Sympathie / Empathie, Persönlichkeit des Arztes, Autonomie) und fachlich therapeutische Aspekte der Beziehung (z. B. fachliche Kompetenz des Arztes, Umgang mit therapeutischen Entscheidungen). Innerhalb dieser Gliederung ließ sich eine Gewichtung der Faktoren erkennen. Besonders wichtig war den Patienten die notwendige „Passung“ zwischen Arzt und Patient. Eine Patientin stellte sogar einen Vergleich zu einer Liebesbeziehung her, in der man ja auch einfach das Glück brauche, „den Richtigen zu finden“ (Patientin 8). Es schien sich hier zunächst um abstrakte Begriffe wie etwa Sympathie und Antipathie zu handeln, deren Ursprung nur schwer zu erfassen ist. Allerdings spielten auch Aspekte, die sich besser konkretisieren lassen und direkte Verbesserungsansätze möglich machen, eine Rolle. Hierzu zählt die gewünschte Empathie, die Tiefe der persönlichen Beziehung und Respekt vor der Autonomie des Patienten. Die in dieser Untersuchung befragten Patienten wollten in der langfristigen Therapie in Entscheidungen eingebunden sein und diese auch gemäß ihrer individuell empfundenen Bedürfnisse beeinflussen können. Weiter unten (in Abschnitt 6.4 wird daher das Shared Decision Making-Modell (CHARLES et al. 1997) als eine mögliche Vorgehensweise auch für die Betreuung und Behandlung schizophrener Patienten diskutiert.

Ein wesentliches Bedürfnis der befragten Patienten war, dass der Arzt mit innen mitfühlen, Hoffnung vermitteln und sie auf emotionaler Ebene ernst nehmen kann und will - Eigenschaften, die man (neben weiteren) unter dem Begriff Empathie zusammenfassen kann und die sowohl verbal als auch non-verbal kommuniziert werden. Einige Patienten dieser Untersuchung wünschten sich mehr Interesse an ihrer psychischen Erkrankung und die Bereitschaft von Seiten der Hausärzte, diese auch zu thematisieren (Patienten 4, 6, 8). Sie wollten ernst genommen werden und Menschlichkeit und Freundlichkeit erfahren (Patienten 
17, 22, 19). Demgegenüber stehen die Ergebnisse von MCCABE et al. (2002). Durch Konversationsanalyse von Videos der Sitzungen von Psychiatern mit schizophrenen Patienten konnten sie zeigen, dass die Ärzte selbst dann Schwierigkeiten hatten, auf die Sorgen der Patienten zu ihrer Erkrankung einzugehen, wenn diese Themen direkt von den Patienten angesprochen wurden. Dies führte zu Spannungen zwischen den Gesprächspartnern. CARR (1997) hebt hervor, dass die von den Patienten gewünschten Eigenschaften vorwiegend bei Hausärzten gefunden werden, die eine gut ausgebildete Gesprächskompetenz haben, über die Fähigkeit verfügen, Interesse und Betroffenheit zu zeigen, und bereits ein Interesse für psychosoziale Probleme haben. Dies unterstreicht nochmals die Bedeutung und Notwendigkeit dieser hausärztlichen Basisqualifikationen. Eine Stärkung dieser Fähigkeiten speziell für die Versorgung psychisch Kranker könnte eventuell in Form eines Kooperationsmodells von psychiatrischen Kliniken und Ambulanzen mit interessierten Hausärzten geschehen. So könnten zum einen die Ärzte die gewünschte fachliche Unterstützung erhalten (FALLOON et al. 1996), zum anderen könnte man die Patienten gezielt an diese Ärzte verweisen. Ein solcher Rahmen könnte wahrscheinlich auch dazu beitragen, die Kommunikation zwischen Hausarzt und Psychiater zu verbessern (BINDMAN et al. 1997, SPIESSL und CORDING 2000).

Veränderungen der Kommunikation innerhalb der therapeutischen Beziehung sind allerdings mit deutlich geringerem Aufwand möglich. Eindrücklich zeigten VAN Os et al. (2004) dies in einer Erhebung über den Einsatz der Two-Way Communication Checklist. Durch die Anwendung dieses Instruments, mit dem sich die Patienten unmittelbar vor dem Arztbesuch auf das Gespräch vorbereiteten, konnte die Zufriedenheit der schizophrenen Patienten bezüglich der Kommunikation mit ihrem Arzt signifikant gesteigert werden. In dieser Gruppe wurde - im Vergleich zur Kontrollgruppe - die Therapie deutlich häufiger an die Bedürfnisse der Patienten angepasst; auch das Verhalten des Arztes veränderte sich durch den Einsatz der Checkliste. Es scheint sich hier also um ein verhältnismäßig einfaches und doch wirkungsvolles Instrument zu handeln, dessen Einsatz im klinischen Altag durchaus zu diskutieren ist.

\subsection{Auswirkung der Arzt-Patient-Beziehung auf die Compliance}

Die von uns befragten Patienten schätzen eine gute Arzt-Patient-Beziehung als relevant für die Compliance ein, worauf auch vorangegangene Studien hinweisen (FLEISCHACKER et al. 2003, AQUILA et al. 1999). So kamen KAMPMAN und LEHTINEN (1999) in einem Review von 67 Artikeln über Compliance bei psychotischen Erkrankungen zu dem Schluss, dass die therapeutische Beziehung signifikanten Einfluss auf die Compliance hatte. Die Studien ließen aber keinen Rückschluss darauf zu, welche Qualitäten einer Beziehung sich auf die Compliance auswirken und in welcher Form dies geschieht. Hier können Aspekte, die von unseren Interviewpartnern berichtet wurden, weiterführend sein. Demnach spielte vor allem die Entscheidungsfindung in der therapeutischen Beziehung eine besondere Rolle. 
Die Patienten legten großen Wert darauf, bei therapeutischen Entscheidungen einbezogen zu werden und ihre Bedürfnisse berücksichtigt zu sehen; viele behielten sich die letztendliche Entscheidung vor. Daraus ergab sich auch die Erwartung, dass Ärzte und Therapeuten ihre Therapievorschläge transparent machen, d. h. in einer für die Patienten gut verständlichen und zu verarbeitenden Weise Information und Argumente zur Verfügung stellen. Die befragten Patienten differenzierten hier allerdings durchaus zwischen Dauerbehandlung und Maßnahmen im akuten Schub. In der Situation der akuten Psychose oder einer drohenden Psychose hielten einige Patienten (Patienten 17,18) ein autoritäres Vorgehen des Arztes für durchaus gerechtfertigt und zum Teil auch notwendig.

Aus den Interviews kann - Krankheitseinsicht vorausgesetzt - eine Hypothese über den Zusammenhang zwischen der Beteiligung des Patienten an der Entscheidungsfindung und der Compliance generiert werden Abbildung 6.2. So scheint die Compliance insbesondere dann hoch, wenn beide Personen etwa zu gleichen Teilen an der Entscheidung beteiligt sind und somit die Entscheidung gemeinsam tragen. Gewinnt hingegen eine Person ein Übergewicht, scheint dies eher hinderlich. In den Extrempositionen kann dies bedeuten, dass der Patient völlig selbständig entscheidet und auch die Ratschläge des Arztes nicht mehr annimmt, oder, auf der entgegen gesetzten Seite, dass er sich einem autoritären Stil des Arztes widersetzt, indem er die Medikamenteneinnahme verweigert. Sicherlich ist dieser Ansatz zunächst nur ein Modellentwurf für eine weitere, systematische Überprüfung.
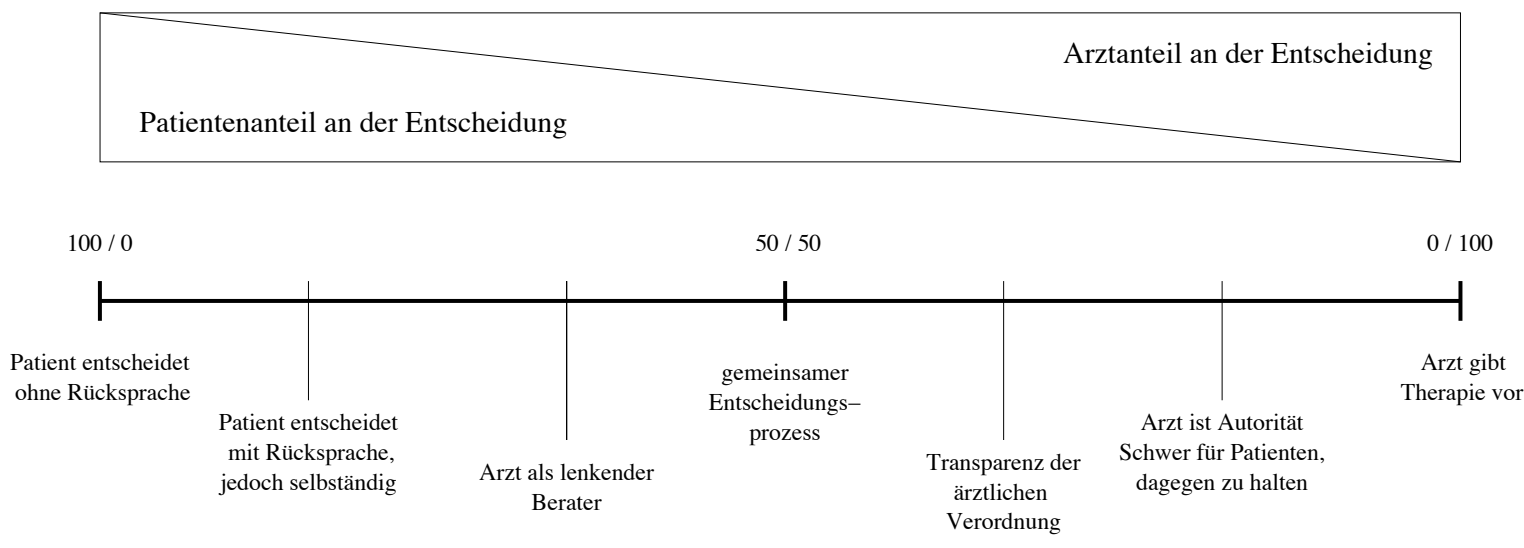

Abbildung 6.2: Beteiligung am therapeutischen Entscheidungsprozess

In der Fachliteratur stößt man hier auf das Shared Decision Making-Modell (engl. für: geteilte Entscheidungsfindung), welches sich in der somatischen Medizin bereits etabliert hat und sogar in entsprechende Lehrbücher aufgenommen wurde (HIMMEL et al. 2004). Es beinhaltet eine aktive Beteilung von Arzt und Patient am Entscheidungsprozess (CHARLES et al. 1997): zum einen stellt der Arzt eine Vielfalt an Informationen über Therapiemöglichkeiten sowie seine persönlichen Ratschläge zur Verfügung, zum anderen stellt der Patient seine Vorstellungen und Präferenzen dar. Die Entscheidung soll dann im Einvernehmen gefällt werden.

In einem Review-Artikel über die Anwendung des Shared Decision Making in der Psych- 
iatrie arbeiteten HAMANN et al. (2003) heraus, dass dieses Modell in der psychiatrischen Praxis keine routinemäßige Anwendung finde. Sie zeigten jedoch Beispiele auf, wie in der somatischen Medizin hierdurch Verbesserungen in der Compliance, dem Behandlungserfolg und in der Zufriedenheit der Patienten erzielt werden konnten. Hinzu kam, dass ein großer Anteil vor allem jüngerer Patienten in die Entscheidungsfindung eingebunden sein wollte, auch wenn insgesamt die Wünsche der Patienten bezüglich ihrer Einbindung in die Entscheidung stark variierten. Dies zeigte eine Befragung mittels strukturiertem Interview von 410 Patienten in schottischen Allgemeinarztpraxen (McKINSTRY 2000). In der Studie von HAMANN et al. (2005) äußerten schizophrene Patienten das Bedürfnis, am Entscheidungsprozess teilzunehmen. Im Gegensatz zu der vorliegenden Studie handelte es sich jedoch um Patienten in stationärer Behandlung während eines akuten Schubs. Von ihnen wünschten sich vor allem diejenigen Patienten Beteiligung am Entscheidungsprozess, die bei mangelnder Krankheitseinsicht gegen ihren Willen hospitalisiert waren.

Auch bei den von uns befragten schizophrenen Patienten in ambulanter Behandlung war der Wunsch nach Beteiligung am Entscheidungsprozess wiederholt Thema in den Interviews, zum einen direkt beim Umgang mit Medikamenten in der therapeutischen Beziehung, zum anderen bei der Kommunikation und Therapie im Allgemeinen. Jenes Interesse an Beteiligung an der therapeutischen Entscheidung, wie sie in der somatischen Medizin gewünscht wird und mit positiven Veränderungen einhergeht, wurde also auch von einigen Personen dieser Gruppe psychisch schwer Kranker als erstrebenswert angesehen. Daraus ergibt sich, dass von Seiten der Betreuenden dieser Patienten eine größere Bereitschaft gefordert ist, auf die individuellen Bedürfnisse des einzelnen einzugehen und dem Patienten angemessen Informationen und Beratung zur Verfügung zu stellen. Der Respekt vor der Autonomie des Patienten ist Vorraussetzung für die Anwendung des Shared Decision Making-Modells, und der mehrfach geäußerte Wunsch danach in den vorliegenden Interviews, legt den Einsatz dieses Konzepts auch bei schizophrenen Patienten nahe. Ausreichendes Datenmaterial zu der Frage, ob durch die Anwendung auch eine objektivierbare Verbesserung der Therapie erreicht werden kann, liegt - wie FENTON (2003) betont - noch nicht vor. Hier könnten weiterführende Untersuchungen ansetzen.

Die Bereitstellung von Informationen - essentieller Bestandteil einer gemeinsamen Entscheidungsfindung - könnten eventuell auch psychosoziale Beratungsstellen, fachlich geschultes Pflegepersonal in den Ambulanzen psychiatrischer Kliniken oder Informationsbroschüren zum Teil übernehmen, so dass der Patient bereits vorbereitet in das Gespräch mit dem behandelnden Arzt geht. Auf diese Weise können Hausärzte zeitlich entlastet werden. In einer Studie in englischen Allgemeinarztpraxen konnten LITTLE et al. (2004) zeigen, dass insbesondere bei kurzen Konsultationszeiten die Zufriedenheit der Patienten im Bezug auf die Kommunikation signifikant gesteigert werden konnte, wenn sie sich mit Hilfe von Broschüren auf den Arztbesuch vorbereiteten. Aus der genannten Studie waren jedoch explizit Patienten mit schweren psychiatrischen Erkrankungen wie Schizophrenie ausgeschlossen. Es wäre also interessant herauszufinden, ob auch schizophrene Patienten Informationsma- 
terial als positive Kompensation für kurze Konsultationszeiten empfinden.

\subsection{Persönliche Erfahrungen und Einstellungen als weitere Einflussfaktoren von Compliance}

In den Interviews schilderten die Patienten, welche Faktoren sie persönlich bei der Entscheidung beeinflussen, ob sie ihre Medikamente einnehmen oder nicht. Eine Rolle spielten für sie dabei unter anderem die unerwünschten Wirkungen des Medikaments. Diese verloren allerdings bei großem erlebten Nutzen im Sinne einer „Kosten“-Nutzen-Abwägung an Relevanz.

Die befragten Patienten sprachen aber vor allem den weit schwerer messbaren Faktoren, wie ihren eigenen Erfahrungen mit und Einstellungen gegenüber Psychopharmaka, eine besondere Bedeutung zu. Viele betonten, dass in erster Linie ihre eigenen Erfahrungen mit Medikamententherapie und (initialer) Noncompliance bei der Entscheidung für die Einhaltung der aktuellen Therapie ausschlaggebend gewesen seien. Dabei handelte es sich zum einen um positive Erfahrungen, etwa der Möglichkeit, durch Psychopharmaka ein weitgehend normales Leben führen zu können, andererseits trugen Erlebnisse mit Rückfällen, die als Folge von Noncompliance auftraten zur Therapietreue (und auf diese Art zur Vermeidung von Rückfällen) bei. Mehrfach ging der Erkenntnis jedoch ein wiederholter Kreislauf aus Therapie, Noncompliance und Rückfall voraus (Patienten 2, 8).

In einer Studie zu den subjektiven Gründen für Compliance und Noncompliance (LÖFFLER et al. 2003) befragten die Autoren 307 Patienten mit der Diagnose Schizophrenie. Mittels der ROMI-Skala, die auf Einstellungen und des Verhalten fokussiert, wurden die von den Patienten angegebenen Gründe für Compliance und Noncompliance standardisiert ausgewertet. Die häufigsten Gründe für Compliance waren demnach der Wunsch, dem Auftreten von psychotischen Symptomen und einem Rückfall vorzubeugen (genannt von $88 \%$ der Patienten) sowie die wahrgenommene Verbesserung des allgemeinen Wohlbefindens und Gesundheitszustands (79\%). Die Autoren ließen jedoch die Frage offen, wie sich positive und negative Einstellungen bilden. Hier kann unsere Studie einen Ansatz bieten. So werden wiederholt die eigenen Erfahrungen direkt in Zusammenhang mit der persönlichen Einstellung gegenüber Medikamenten gebracht. Mehrere Patienten beschrieben, wie sich als Folge erlebter Rückfälle oder negativer Erlebnisse, die durch die Krankheit ausgelöst wurden, wie etwa Verlust der Arbeitsstelle (Patient 14) oder familiäre Zerwürfnisse (Patient 17), ihre Einstellungen gegenüber Medikamenten verändert hatten. Der Ablehnung der Medikamente wich einer gewissen Akzeptanz, die Patienten nahmen sie als das „kleinere Übel“ an.

Eine Patientin (35 Jahre) machte einen bemerkenswerten Vorschlag, wie es trotz Ablehnung des ärztlich verordneten Therapieplans nicht zum Abbruch der therapeutischen Beziehung kommen muss, sondern - im Gegenteil - die Beziehung gefestigt werden kann. Sie betonte die Wichtigkeit, „dass die Betreuung da ist, weil der Patient setzt die Tabletten so 
oder so ab, und dann sollte der Arzt wenigstens sagen: Okay, dann aber mit meiner Hilfe und mehreren Besuchen in kürzeren Abständen" (vgl. Abschnitt 5.4.7, Q 19:23). Auf diese Art könnte es den Patienten eventuell leichter fallen, in einem geschützten Umfeld wichtige Erfahrungen zu sammeln und den oben genannten Kreislauf abzukürzen. Sicherlich begibt man sich hier auf eine Gratwanderung, da die Empfehlung eines von international akzeptierten Richtlinien (etwa zur antipsychotischen Erhaltungstherapie) abweichenden Therapiekonzepts ethisch bedenklich ist.

\subsection{Kritische Bilanz der methodischen Umsetzung}

Um das bisher wenig untersuchte Themengebiet der hausärztlichen Versorgung aus Sicht schizophrener Patienten zu beleuchten, wurde ein qualitatives Studiendesign gewählt (semistrukturierte Interviews). Der offene und zurückhaltende Kommunikationsstil von Seiten der Interviewer stellte die Patienten in den Mittelpunkt und ermöglichte innen somit, Akzente zu setzen. Die erhobenen Daten zeigen ein facettenreiches Bild der Situation schizophrener Patienten in ambulanter Behandlung und erfüllen somit den Anspruch der Heterogenität des Datenmaterials. Andererseits stand diese Offenheit aber manchmal im Kontrast zur gezielten thematischen Datenerhebung. Die Patienten schweiften in Aspekte ihrer Krankheitsgeschichte ab, für die ein Mitteilungsbedürfnis zu bestehen schien. Es war dann trotz gezielter Nachfragen manchmal schwierig, wieder zum Interessengebiet der Untersuchung zurückzufinden (z. B. Patienten 1, 17). Die Interviews variierten daher in ihrer Verwertbarkeit für die Fragestellung der Arbeit.

Die vorliegende Arbeit wurde unter Berücksichtigung der Gütekriterien qualitativer Forschung (STEINKE 2000, S. 324 f.) geplant und durchgeführt. Das Ziel einer intersubjektiven Nachvollziehbarkeit von Prozess und Interpretation wurde durch die kontinuierliche Dokumentation mittels spezieller Software unterstützt. Zudem erfolgten die Anpassung der Methode an die Fragestellung und die Auswertung im ständigen Dialog innerhalb eines multiprofessionellen Teams (eine Medizinerin, eine Dipl.-Psychologin, eine Anthropologin).

Der empirischen Verankerung der generierten Hypothesen diente die Anwendung einer regelgeleiteten Methode, orientiert an SCHMIDT C (2000), sowie die analytische Induktion (STEINKE 2000, S. 325) und Verifikation anhand von Textbelegen im Datenmaterial. Dies spiegelt sich vor allem auch in der angewandten zirkulären Strategie wider (vgl. Abschnitt 4.2). Es sei an diesem Punkt betont, dass die empirische Verankerung der Hypothesen unbedingt von einer empirischen Validierung (im Sinne quantitativer Methoden) unterschieden werden muss. Eine Verifizierung der Aussagen mittels einer quantitativ standardisierten Methode wäre ein nächster Schritt.

Als weiteres Gütekriterium qualitativer Forschung nennt STEINKE (2000, S. 326 f.) die angemessene Indikation des Forschungsprozesses. Inwieweit die Untersuchung hinsichtlich der Methoden- und Strategienwahl dieses Kriterium erfüllt, wurde bereits in Kapitel 4 darge- 
stellt: Die deskriptive Zielsetzung der Studie sowie die sozialen, interaktiven und weitgehend unbekannten Elemente des Forschungsgegenstandes sind gültige Indikationen für ein qualitatives Forschungsdesign (BERKWITS und ARONOWITZ 1995, WRIGHT et al. 1998, FLICK 2000).

Nicht zuletzt erfüllt die Untersuchung auch das Kriterium der Relevanz. Anknüpfend an zum Teil schon untersuchte Phänomene (wie etwa dem Zusammenhang von Arzt-PatientBeziehung und Compliance) eröffnet sie neue Perpektiven, wie etwa die bis heute weitgehend unbekannte Sichtweise der Patienten.

Eine Schwierigkeit war die Rekrutierung der schizophrenen Patienten. Auch durch das Ausschöpfen unterschiedlichster Methoden war die Resonanz auf die Befragung sehr zurückhaltend, was direkten Einfluss auf die methodische Umsetzung hatte. Das vorgesehene Theoretical Sampling nach STRAUSS und CORBIN (1990) war unter diesen Umständen nicht möglich. Bei diesem Vorgehen wird gezielt nach Datenmaterial gesucht, das sich von bereits vorliegendem unterscheidet. Unter diesen Gesichtpunkten wird im Wechsel mit der Auswertung der Daten der jeweils nächste Interviewpartner ausgesucht, bis sich bei der Analyse keine neuen Aspekte mehr finden lassen. Erst dann ist die Datenerhebung abgeschlossen. In dieser Untersuchung wurde eine „Sättigung“ des erhobenen Datenmaterials dadurch erreicht, dass sich Phasen der Datenerhebung und -erfassung bewusst abwechselten. So konnten immer wieder die relevanten Themen erfasst und in die Gestaltung der folgenden Interviews integriert werden. Auf diesem Weg konnte die Bandbreite der angesprochenen Themen dargestellt werden, so dass der Anspruch der Sättigung, nicht aber der Repräsentativität erhoben werden kann.

Weiterhin Schwierigkeiten bereitete die Umsetzung der Critical Incident Technique (FLANAGAN 1954). Diese Methode sollte zum Einsatz kommen, indem die Patienten zum Abschluss des Interviws aufgefordert wurden, sich an den letzten Besuch jeweils bei ihrem Hausarzt und bei ihrem Facharzt zu erinnern. Von Interesse für die Untersuchung waren positive oder negative Erlebnisse, wie etwa bestimmte Verhaltensweisen des jeweiligen Arztes. Hierdurch sollte an realen Beispielen nach Verbesserungsmöglichkeiten gesucht werden. Den befragten Patienten fiel es jedoch sichtlich schwer, sich diese konkreten Situationen ins Gedächtnis zu rufen, vielmehr folgten auf diese Frage eher allgemein gehaltene Antworten (z. B. „So wie immer“) oder aber die Aussage, sich nicht erinnern zu können. Die Critical Incident Technique wurde daher im Verlauf der Erhebung verlassen.

\subsection{Schlussfolgerung}

Die Patientenperspektive darf man als wesentliche Stärke dieser Arbeit betrachten. In den meisten vorangegangenen Untersuchungen, insbesondere zur Verbesserung von Compliance, wurde das Thema vornehmlich aus der Sicht des Arztes beleuchtet. Neben zahlreichen älteren Artikeln und Metaanalysen (HAEBERLIN THOMAS et al. 1992, FENTON et al. 1997, 
HORNUNG et al. 1998 zeigt dies nochmals ein aktueller Übersichtsartikel, der ärztliche Maßnahmen zur Verbesserung der Compliance aufführt (RABOVSKY und STOPPE 2006). Auch in Bezug auf den zweiten wichtigen Untersuchungsbereich der vorliegenden Arbeit, der Ausbildung einer tragfähigen therapeutischen Beziehung, gab es nur wenige Erkenntnisse über die Sichtweise der Patienten (FRANK und GUNDERSON 1990). An diesem Punkt setzte die vorliegende Arbeit an.

Der Hausarzt nimmt in der ambulanten Behandlung schizophrener Patienten eine wichtige Rolle ein. Dies bezieht sich zunächst auf die Aufgaben als „Körperarzt“, die weitgehend mit einem nicht psychisch kranken Patientenkollektiv deckungsgleich sind. Daneben übernimmt er auch Funktionen bei Behandlung und „Management“ der psychischen Erkrankung. Er führt die vom Facharzt verordnete Medikamententherapie fort, koordiniert in einer Gatekeeper-Funktion die Behandlung und die sozialen Ressourcen des Patienten (insbesondere bei Erkrankungsbeginn), ist wichtige Informationsquelle für den Patienten und seine Angehörigen und ist zudem oft auch Ansprechpartner für alltägliche und psychische Probleme. Die Stärken der hausärztlichen Versorgung liegen hier vor allem in der von den Patienten wahrgenommenen „Normalität“ und, insbesondere zu Beginn der Erkrankung, im Vertrauensvorschuss. Im Vergleich zum Facharzt ist die Furcht vor Stigmatisierung beim Hausarzt geringer und die Schwelle, inn aufzusuchen, scheint niedriger zu sein. Die Studie unterstreicht die Notwendigkeit hausärztlicher Kompetenz in der Erkennung von Frühzeichen einer Psychose sowie von Fachkenntnissen im Bereich von Psychopathologie, Psychopharmakologie und ergänzenden Therapiemöglichkeiten zur Förderung dieser hausärztlichen Stärken. Eine Verbesserung der Kooperation mit psychiatrischen Fachkollegen und ggf. eine Stärkung dieser Bereiche in der Weiterbildung zum Allgemeinarzt scheinen vor diesem Hintergrund erstrebenswert.

Die Patienten dieser Studie äußerten wiederholt den Wunsch nach vermehrter Beteiligung am therapeutischen Entscheidungsprozess im Sinne des Shared Decision Making, das im Bereich der Psychiatrie noch keinen routinemäßigen Einsatz findet. Grundlage hierfür wäre eine tragfähige therapeutische Beziehung, die auch einen nicht unerheblichen Einfluss auf die Compliance hat. Die aus den Interviews erarbeiteten Gütekriterien der Arzt-PatientBeziehung gliedern sich in strukturelle Merkmale (z. B. Dauer und Kontakthäufigkeit der Beziehung, Infrastruktur), Passung zwischen Arzt und Patient (z. B. Sympathie / Empathie, Persönlichkeit des Arztes, Autonomie) und fachlich therapeutische Aspekte der Beziehung (z. B. fachliche Kompetenz des Arztes, Umgang mit therapeutischen Entscheidungen). Kann auf diesen Ebenen eine tragfähige therapeutische Beziehung etabliert werden, in der die Autonomie des (krankheitseinsichtigen) Patienten in Langzeitbehandlung gewahrt bleibt, wäre dies auch zur Förderung der Compliance wünschenswert. Hieraus ergeben sich Fragestellungen für weitere Untersuchungen zu Art und Ausmaß der Anwendbarkeit dieses Modells sowie zur Frage des tatsächlichen Nutzens für die Patienten. 


\section{Zusammenfassung}

\section{Ausgangspunkt}

Schizophrene Patienten werden ambulant durch Psychiater und in den meisten Fällen auch durch Hausärzte betreut. Daher sollten Merkmale und Aufgabenschwerpunkte der hausärztlichen Betreuung untersucht werden, mit besonderer Beachtung der Arzt-Patient-Beziehung und ihrer Auswirkung auf die Medikamenten-Compliance.

\section{Methode}

In Göttingen, Hildesheim, Kassel und Gießen wurden Interviews mit schizophrenen Patienten geführt. Die Patienten wurden gebeten, die Bedeutung der verschiedenen Ärzte für ihre Behandlung, die Merkmale einer guten therapeutischen Beziehung und die Einflussfaktoren auf Compliance aus ihrer Perspektive zu schildern. Die Interviews wurden mit qualitativen Methoden in Anlehnung an den Editing Analysis Style nach Crabtree und Miller (CRABTREE und MILLER 1992) analysiert.

\section{Ergebnisse}

Es wurden 20 Interviews geführt und ausgewertet. Da Hausärzte und Ansprechpartner in Selbsthilfegruppen die Patienten für uns rekrutierten, ist die Zahl der Verweigerer nicht verlässlich zu schätzen. Die Patienten äußerten genaue Vorstellungen von den Zuständigkeitsbereichen des jeweiligen Arztes: der Hausarzt überwiegend für die körperliche und der Facharzt für die seelische Gesundheit. Dennoch nimmt der Hausarzt auch in der psychiatrischen Behandlung eine definierte Rolle ein, etwa indem er bei Erkrankungsbeginn als Gatekeeper fungiert oder aber im Verlauf die vom Facharzt empfohlene Therapie fortführt. Patientenbezogene Faktoren (z. B. persönliche Einstellungen und Erfahrungen), Beziehungsaspekte (z. B. Entscheidungsprozesse in der therapeutischen Beziehung) und medikamentengebundene Faktoren (z. B. unerwünschte Medikamentenwirkungen) beeinflussten nach Meinung der Befragten die Compliance. Eigene Erfahrungen mit positiven Medikamentenwirkungen (z. B. die Vermeidung eines akuten Schubs oder das Erleben von Normalität) wurden am häufigsten als stabilisierender Einfluss auf die Compliance genannt. Aber auch negative Folgen von Noncompliance (z. B. Hospitalisation oder Verlust des Arbeitsplatzes) waren manchmal ein wichtiger Beweggrund für die aktuelle Therapietreue. Die Ausbildung einer 
guten therapeutischen Beziehung war nach Darstellung der Patienten abhängig von: strukturellen Aspekten (z. B. Dauer der Beziehung, Häufigkeit des Kontakts), kommunikativen Aspekten (z. B. Art und Umfang des Informationsaustausches), fachlichen Aspekten (z. B. wahrgenommener Fachkompetenz des Arztes), sozialen Aspekten (z. B. Einbindung von Angehörigen in das Therapiekonzept) und persönlichkeitsbezogenen Aspekten (z. B. Passung von Arzt und Patient). Vom Hausarzt wünschten die Patienten eine gute psychiatrische Fachkenntnis, Empathie sowie soziale und kommunikative Qualitäten. Außerdem wollten sie bei therapeutischen Entscheidungen beteiligt werden.

\section{Diskussion}

Hausärzte nehmen in der Behandlung schizophrener Patienten in erster Linie die Rolle eines „Körperarztes“ ein. Bei Erkrankungsbeginn, im Bereich der Früherkennung, der Verordnung (oder Weiterververordnung) antipsychotischer Medikamente und der begleitenden psychosozialen Patienten- und Familienbetreuung sind sie aber für viele Patienten auch medizinischer Fachmann und kompetenter Ansprechpartner. Daraus ergibt sich für Hausärzte die Notwendigkeit profunder Kenntnisse von Früherkennungszeichen, Psychopathologie und Psychopharmakologie sowie einer funktionierenden Kooperation mit psychiatrischen Fachkollegen. Von den die Compliance beeinflussenden Faktoren maßen die Patienten ihrer persönlichen Erfahrung die größte Bedeutung zu. Auch wenn manchmal gerade einschneidende negative Folgen von Noncompliance Therapietreue nach sich zogen, wäre es ethisch bedenklich, Patienten im Rahmen der Behandlung aus strategischen Gründen zu Noncompliance zu ermutigen und damit von internationalen Richtlinien der Pharmakotherapie abzuweichen. Der wiederholt von den Patienten geäußerte Wunsch nach stärkerer Einbindung in den therapeutischen Entscheidungsprozess legt die (ggf. modifizierte) Anwendung des Shared Decision Models auch bei der ambulanten Behandlung schizophrener Patienten nahe. 


\section{Literaturverzeichnis}

AвHolz HH, Kochen MM: Definition der Allgemeinmedizin. In: Kochen MM (Hrsg.): Allgemeinmedizin und Familienmedizin. Thieme, Stuttgart 2006, 502-506.

Albus M (1995): Zum Problem der Medikamenten-Compliance bei Schizophrenen Patienten. Psychiatr Prax 22, 221-222.

American PSychiatric Association: DSM-IV. Diagnostic and Statistical Manual of Mental Disorders (4th ed.). American Psychiatric Association, Washington D. C. 1994.

Andor ES, Lauber C, Ludewig K, Braun-Scharm H, Umbricht DS (2005): General practitioners and schizophrenia: Results from a Swiss Survey. $\operatorname{Br} J$ Psychiatry 187, 274-81.

Aquila R, Weiden PJ, Emanuel M (1999): Compliance and the rehabilitation alliance. $J$ Clin Psychiatry 60 (Suppl. 19), 23-27.

AufEnANGer S: Qualitative Analyse semi-strukturierter Interviews - Ein Werkstattbericht. In: Garz D, Kraimer K (Hrsg.): Qualitativ-empirische Sozialforschung: Konzepte, Methoden, Analysen. Westdeutscher Verlag, Opladen 1991, 35-59.

BAG B, YILMAZ S, KIRPINAR I (2005): Factors influencing social distance from people with schizophrenia. Int J Clin Pract 60, 289-294.

BebiIngton PE (1995): The content and context of compliance. Int Clin Psychopharmacol 9 (Suppl. 5), 41-50.

BECKER MH, MAINMAN LA (1975): Sociobehavioral determinants of compliance with health and medical care recommendations. Med Care 13, 10-24.

BERKWITS M, ARonowitz R (1995): Different questions beg different methods. J Gen Intern Med 10, 409-410.

Bindman J, Johnson S, Wright S, Szmukler G, Bebbington P, Kuipers E, ThorniCROFT G (1997): Integration between primary care and secondary services in the care of the severely mentally ill: patient's and general practitioner's views. Br J Psychiatry $171,169-174$.

Birchwood M, Smith J, Cochrane R, Wetton S, Copestake S (1990): The Social Fuctioning Scale: The development and validation of a new scale for social adjustment for use in family intervention programs withs schizophrenic patients. $B r J$ Psychiatry 157, 853-59. 
BRADLEY CP (1992): Uncomfortable prescribing decisions: a critical incident study. BMJ 304, 294-296.

BRown S (1997): Excess mortality of schizophrenia. A meta-analysis. Br J Psychiatry 171, 502-508.

BUCHANAN A (1992): A two-year prospective study of treatment compliance of patients with schizophrenia. Psychol Med 22, 787-797.

BÖKER W (1988): Der Schizophrene Patient in hausärztlicher Betreuung. Hexagon 16, $13-18$.

CARR VJ (1997): The role of the general practitioner in the treatment of schizophrenia: general principles. Med J Aust $\underline{166}, 91-94$.

CASPAR ES, REGAN JR (1993): Reasons for admission amongst six profile subgroups of recidivists of inpatient services. Can J Psychiatry $\underline{38}, 657-661$.

Charles C, Gafini A, Whelan T (1997): Shared decision-making in the medical encounter: what does it mean? (or it takes at least two to tango). Soc Sci Med 44, 681-692.

Christensen B, Sorensen HT, MABeck CE (1989): Differences in referral rate from general practice. Fam Pract $\underline{6}, 19-22$.

CoRRIGAN P (2004): How does stigma interfere with mental health care. Am Psychol 59 , 614-25.

Couture SM, Roberts Dl, Penn Dl, Cather C, Otto MW, Goff D (2006): Do baseline client characteristics predict the therapeutic alliance in the treatment of schizophrenia. J Nerv Ment Dis 194, 10-14.

Crabtree F, Miller WL: Doing Qualitative Research Vol. 3, Research Methods for Primary Care. Sage Publications, Newbury Park 1992.

de Cruppe W, Müller A, Herzog W, W Eıch (2006): Psychosomatische Versorgungspraxis und Kooperationsbedarf aus Sicht niedergelassener Ärzte und Psychologen. Psychol Psychother Med 트, 299-306.

DGPPN (2005): Leitlinien der Deutschen Gesellschaft für Psychiatrie, Psychotherapie und Nervenheilkunde: Schizophrenie. AWMF online: AWMF Leitlinienregister Nr. 038/009. verfügbar unter: http://www.uni-duesseldorf.de/AWMF/11/038-009.htm. letzter Zugriff 24.4.2007.

DGPPN (Deutsche Gesellschaft für Psychiatrie, Psychotherapie und NerVENHEILKUNDE): Die Behandlung psychischer Erkrankungen in Deutschland. Springer, Berlin 1997.

Dilling H, MOMbOUR W, Schmidt MH (HRSG.): Internationale Klassifikation psychischer Störungen. ICD-10 Kapitel V . (Klinisch-diagnostische Leitlinien) (5. rev. ed.). Hans Huber, Bern 2004. 
DIMATTEO MR (2004): Variations in patients' adherence to medical recommondations - a quantitative review of 50 years of research. Med Care 42 (3), 200-209.

EICH PE (1990): Der praktische Arzt in sozialpsychiatrischen Konzepten der Schizophreniebehandlung. Ther Umschau $\underline{3}, 225-232$.

Emmus (1999): Critical Incident Technique. verfügbar unter: http://www.ucc.ie/hfrg/ emmus/methods/cit.html. letzter Zugriff 24.4.2007.

EPSTEIN LH (1984): The direct effects of compliance on health outcome. Health Psychol 3, 385-393.

FALLOON IR, NG B, BENSEMANN C, KYDD RR (1996): The role of the general practitioner in mental health care: a survey of needs and problems. NZ Med J 109, 34-36.

FENTON WS (2003): Shared decision making: a model for the physician-patient relationship in the 21st century? (Editorial). Acta Psychiatr Scand 107, 401-402.

FEnton WS, BLyLER CR, HeINSSEN RK (1997): Determinants of medication compliance in schizophrenia: empirical and clinical findings. Schizophr Bull 23, 637-651.

Fernando ML, Cooper AJ, Cernovsky Z, Bailey C, Velamoor VR (1990): The impact of the amended Ontario Mental Health Act (Bill 7) on patients discharging themselves from hospital against medical advice (AMA): a preliminary study. Can J Psychiatry 35, 77-91.

FIELDING NC, LEE RL: Using computers in qualitative research. Sage Publications, London 1991.

Flanagan JC (1954): The Critical Incident Technique. Psychol Bull 51, 327-358.

Fleischacker WW, OeHL MA, Hummer M (2003): Factors influencing compliance in schizophrenia patients. J Clin Psychiatry 64 (Suppl. 10), 10-13.

FLICK U: Design und Prozess qualitativer Forschung. In: Flick U, von Kardorff E, Steinke I (Hrsg.): Qualitative Forschung. Ein Handbuch. Rowohlt, Hamburg 2000, 252-265.

FRANK AF, GUNDERSON JG (1990): The role of the therapeutic alliance in the treatment of schizophrenia. Relationship to course and outcome. Arch Gen Psychiatry 47, 228-236.

GaEbel W (1998): Critical issues in the treatment of schizophrenia. Int Clin Psychopharmacol 13 (Suppl. 3), 1-6.

Grol R, Wensing M, Mainz J, Ferreira P, Hearnshaw h, Huortdahl P, Olesen F, Ribacke M, Spenser T, SzecsenyI J (1999): Patients' priorities with respect to general practice care: an international comparison. Fam Pract 16, 4-11.

HAASEN C, STARK FM (1997): Subjektive Kriterien Schizophrener bei der Arztwahl in der ambulanten Betreuung. Psychiatr Prax 24, 74-76.

HAASEN C, Bergländer T, StARK FM (1993): Epidemiologische Aspekte bei der Betreuung Schizophrener durch Allgemeinärzte. Psycho 19, 12-21. 
HAEBerlin Thomas B, ERnSt C, ERnSt K (1992): Wie erreicht man Compliance? Zur Nachbehandlung Psychischkranker nach dem Klinikaustritt. Nervenarzt 63, 442-443.

HAMANN J, LEUCHT S, KISSLING W (2003): Shared decision making in psychiatry. Acta Psychiatr Scand 107, 403-409.

Hamann J, Cohen R, Leucht S, Busch R, Kissling W (2005): Do patients with schizophrenia wish to be involved in decisions about their medical treatment? Am J Psychiatry 162, 2382-85.

HaRding G, GantLey M (1998): Qualitative methods: Beyond the cookbook. Fam Pract $15,76-79$.

Haynes RB, Taylor DW, Sackett DL: Compliance Handbuch. Oldenbourg, München 1982.

Hermanns H: Interviewen als Tätigkeit. In: Flick U, von Kardorff E, Steinke I (Hrsg): Qualitative Forschung. Ein Handbuch. Rowohlt, Hamburg 2000, 360-368.

Heszen-Klemens I (1987): Patients non-compliance and how doctors manage this. Soc Sci Med 24, 409-416.

Himmel W, Rogausch A, Kochen MM: Principles of patient management. in Jones $R$, Britten N, Culpepper L et al. (Hrsg.): Primary Medical Care, Vol. 1 Principles and Concepts. Oxford University Press, New York 2004, 228-230.

HJORTDAHL P, LÆERUM E (1992): Continuity of care in general practice: effect on patient satisfaction. BMJ 304, 1287-1290.

HOFFMANN-RIEM C (1980): Die Sozialforschung einer interpretativen Soziologie - Der Datengewinn. Köln Soziol Sozialpsychol 32, 339-372.

HOHL J (2000): Das qualitative Interview. Z Gesundheitswiss $\underline{8}, 142-148$.

Hopf C: Qualitative Interviews ein Überblick. In: Flick U, von Kardorff E, Steinke I: Qualitative Forschung. Ein Handbuch. Rowohlt, Hamburg 2000, 349-360.

Hornung WP, Klingenberg S, Feldmann R, Schonauer K, Schulze Mönking H (1998): Collaboration with drug treatment by schizophrenic patients with and without psychoeducational training: results of a 1-year follow-up. Acta Psychiatr Scand 97, 213-219.

Horvath AO, Greensberg LS (1989): Development and validation of the Working Alliance Inventory. J Couns Psychol 36, 223-33.

Huber GL: Qualitative Analyse. Computereinsatz in der Sozialforschung. Oldenbourg, München 1992.

Kampman O, Lehtinen K (1999): Compliance in psychosis. Acta Psychiatr Scand 100, 167-175. 
Kashner TM, Rader Le, Rodell DE, Beck CM, Rodell LR, Muller K (1991): Family characteristics, substance abuse, and hospitalization patterns of patients with schizophrenia. Hosp Commun Psychiatry 42, 195-197.

KAY SR, FISZBein A, OpPLER LA (1987): The positive and negative syndrome scale (PANSS) for schizophrenia. Schizophr Bull 13, 261-67.

Klingenberg A, Bahrs O, Szecseny J (1996): Was wünschen Patienten vom Hausarzt? Erste Ergebnisse aus einer europäischen Gemeinschaftsstudie. Z Allgemeinmed 72, $180-186$.

Klingenberg A, Bahrs O, Szecseny J (1999): Wie beurteilen Patienten Hausärzte und ihre Praxen? Deutsche Ergebnisse aus einer europäischen Studie zur Bewertung hausärztlicher Versorgung durch Patienten. Z Ärztl Fortbild Qualitätssich 93, 437-445.

KoolWIJK JV: Die Befragungsmethode. In: Koolwijk JV, Wieken-Maiser M (Hrsg.): Techniken der empirischen Sozialforschung. Band 4: Erhebungsmethoden: Die Befragung. Oldenbourg, München / Wien 1974, 9-23.

Kowal S, O'Connell D: Zur Transkription von Gesprächen. In: Flick U, von Kardorff E, Steinke I (Hrsg.): Qualitative Forschung. Ein Handbuch. Rowohlt, Hamburg 2000, 437447.

LACRO JP, DunN LB, DOLdER CR, LeckBANd SG, Jeste DV (2002): Prevalence of and risk factors for medication nonadherence in patients with schizophrenia: a comprehensive review of recent literature. J Clin Psychiatry $\underline{63}, 892-909$.

LAMNEK S: Qualitative Sozialforschung. Band 2: Methoden und Techniken, 3. korrigierte Auflage. Beltz, Weinheim 1995.

Little P, Dorward M, Warner G, Moore M, Stephens K, Senior J, KendRICK T (2004): Randomised controlled trial of effect of leaflets to empower patients in consultations in primary care. BMJ online, published 13 February 2004, doi:10.1136/bmj.37999.716157.44.

LÖFfLeR W, KILIAN R, TOUmı M, ANGeRmeyer MC (2003): Schizophrenic patients' subjective reasons for compliance and noncompliance with neuroleptic treatment. Pharmacopsychiatry $\underline{36}, 105-112$.

MACHLEIDT W: Enstehungsgeschichte, Symptomatik und Verlauf der Schizophrenie. In: Machleidt W, Bauer M, Lamprecht F, Rose HK, Rohde-Dachser C (Hrsg.): Psychiatrie, Psychosomatik und Psychotherapie (6. rev. ed.). Georg Thieme, Stuttgart 1999, 309319.

MARDER SR (2003): Overview of partial compliance. J Clin Psychiatry 64 (Suppl. 16), 3-9.

MAYRING P: Qualitative Inhaltsanalyse. In: Flick U, von Kardorff E, Keupp H, von Rosenstiel L, Wolff S (Hrsg.): Handbuch Qualitative Sozialforschung Grundlagen Konzepte, Methoden und Anwendungen (2. ed.). Psychologie Verlag Union, Weinheim 1995, 209-213. 
McCabe R, Heath C, Burns T, Priebe S (2002): Engagement of patients with psychosis in the consultation: Conversation analytic study. BMJ $\underline{325}, 1148-51$.

MCKINSTRY B (2000): Do patients wish to be involved in decision making in the consultation? A cross sectional study with video vignettes. BMJ $\underline{321}, 867-871$.

MeLLER I, FichtNeR MM, WITZKE W (1989): The role of the general practitioner in the care of mentally disturbed subjects in the general population. Results of the Upper Bavarian Study. Int J Soc Psychiatry 35, 293-302.

Merton RK, Kendall PL : Das fokussierte Interview. In: Hopf C, Weingarten E (Hrsg.): Qualitative Sozialforschung (3. ed.). Klett-Cotta, Stuttgart 1993, 171-201.

Miller WL, Crabtree F (1994): Qualitative analysis: How to begin making sense. Fam Pract Res J 14, 289-297.

Möller HJ, Laux G, A Deister: Psychiatrie (Duale Reihe). Hippokrates, Stuttgart 1996.

Monalto M, HaRRIS P, RosengaRTEN P (1994): Impact of general practitioners referral letters to an emergency department. Aust Fam Physician 46, 69-71.

MunR T: ATLAS.ti 4.2. The Knowledge Workbench. Visual Qualitative Data Analysis of Text, Images, Audio \& Video Materials. Scientific Software Development, Berlin 1997.

Nelson AA, Gold BH, Huchinson RA, BenezRa E (1975): Drug default among schizophrenic patients. Am J Hosp Pharm 32, 1237-1242.

Ngaire K, Buetow S, Mainous AG, Young G, Coster G, Arroll B (2004): Physicianpatient relationship and medication compliance: A primary investigation. Ann Fam Med 2, 455-461.

Ogden J, Andrade J, Eisner M, Ironmonger M, Maxwell J, Muir E, Siriwardena R, THWAITES S (1997): To treat? To befriend? To prevent? Patients' and GPs' views of the doctors role. Scand J Prim Health Care 15, 114-117.

Olfsen M, Lewis-Fernandez R, Weissmann MW, Feder A, Gameroff MJ, PilowSKY D, FUENTES M (2002): Psychotic symptoms in an urban general medicine practice. Am J Psychiatry 159, 1412-1419.

Orleans CT, George LK, Houpt JL, Brodie HK (1985): How primary care physicians treat psychiatric disorders: a national survey of family practitioners. Am J Psychiatry 142, 52-57.

Petermann F: Compliance und Selbstmanagement. Hogrefe, Göttingen 1998.

Philbrick JT, CONELly JE, Wofford AB (1996): The prevalence of mental disorders in rural office practice. J Gen Intern Med 11, 9-15.

Pietschel-Walz G, Bäuml J, Bender W, Engel RR, Wagner M, Kissling W (2006): Psychoeducation and compliance in the treatment of schizophrenia: Results of the Munich Psychosis Information Study. J Clin Psychiatry 67, 443-452. 
RABovsky K, Stoppe G (2006): Prävention und Behandlung von Non-Compliance und Gesamtbehandlungsabbruch. psychoneuro 32, 313-18.

Roberts L, Roalfe A, WiLson S, Lester H (2006): Physical health care of patients with schizophrenia in primary care: a comparative study. Fam Pract, published 24 October 2006, doi:10.1093/fampra/cml054.

Rogers A, Popay J, Williams G, Latham M: Inequalities in Health and Health Promotion: An Insight from the Literature. Health Education Authority, London 1997.

Rosemann T, Wensing M, Rueter G, Szecseny J (2006): Referrals from general practice to consultants in Germany: If the GP is the initiator, patients' experiences are more positive. BMC Health Services Research 6, published 19 January 2006, doi:10.1186/1472-6963-6-5.

SACKETT DL, SNOW JC: The magnitude of compliance and non-compliance. In: Haynes RB, Taylor DW, Sackett DL (Hrsg.): Compliance in Health Care. Johns Hopkins University Press, Baltimore 1979, 11-22.

SARTORIUS N (2002): latrogenic stigma of mental illness. BMJ 324, 1470-71.

SCHMIDT C: Analyse von Leitfadeninterviews. In: Flick U, von Kardorff E, Steinke I (Hrsg.): Qualitative Forschung. Ein Handbuch. Rowohlt, Hamburg 2000, 447-456.

SCHMIDT JU (2004): Schizophrenie. verfügbar unter: http://www.psychiatrie-aktuell. de/disease/detail.jhtml?itemname=schizophrenia. letzter Zugriff 24.4.2007.

SCHMIDT-GRUNERT M (HRSG.): Sozialforschung konkret. Problemzentrierte Interviews als qualitative Erhebungsmethode. Lambertus, Freiburg 1990.

Schmittdiel J, Selby JV, Grumbach K, QuesenberRy CP (1997): Choice of a personal physician and patient satisfaction in a health maintenance organization. JAMA 278, 1596-1599.

SCHOOLER NR (2003): Relapse and rehospitalisation: Comparing oral and depot antipsychotics. J Clin Psychiatry 64 (Suppl. 16), 14-17.

SHERBouRne CD, StuRM R, WeLLS KB (1999): What outcome matters to patients? J Gen Intern Med 14, 357-363.

SPIESSL H, CORDING C (1999): Zusammenarbeit von Hausärzten mit psychiatrischen Einrichtungen aus Sicht der Qualitätssicherung. Z Ärztl Fortbild Qualitätssich 93, 129_ 133.

SPIESSL H, CoRding C (2000): Zusammenarbeit niedergelassener Allgemeinärzte und Nervenärzte mit der psychiatrischen Klinik. Eine Literaturübersicht. Fortschr Neurol Psychiatr 68, 206-215.

STEINKE I: Gütekriterien qualitativer Forschung. In: Flick U, von Kardorff E, Steinke I: Qualitative Forschung. Ein Handbuch. Rowohlt, Hamburg 2000, 319-331. 
StIMSON GV (1974): Obeying doctor s orders: A view from the other side. Soc Sci Med $\underline{8}$, 97-104.

Strauss AL, Corbin J: Grounded Theory. Grundlagen qualitativer Sozialforschung. Beltz-PVU, Weinheim 1996 (engl. Original 1990).

TYLOR S, Bogdan R: Introduction to Qualitative Research Methods. Wiley and Sons, New York 1998.

van Os S, de Abreu R, Hopman J, Wethly K, Liem D, van de Bor M (2004): Evaluation of the Two-Way Communication Checklist as a clinical intervention. Results of a multinational, randomised controlled trial. Br J Psychiatry 184, 79-83.

WILliAmS B (1994): Patient satisfaction: A valid concept? Soc Sci Med 38, 509-516.

World Health Organization: Mental Health: New Understanding, New Hope. WHO Publications, Genf 2001.

World Health Organization: Adherence to Long-term Therapies: Evidence for Action. WHO Publications, Genf 2003.

WRIGHT J, WILLIAMS R, WILKINSON JR (1998): Development and importance of health needs assessment. BMJ $\underline{316}, 1310-1313$. 


\section{Anhänge}

Auf den folgenden Seiten finden sich zum einen die Formulare, die zur Datenerhebung verwendet wurden, zum anderen sind verschiedene Entwicklungsstufen des Leitfadens für die Patienteninterviews, sowie die endgültige Version des Kodierleitfadens und ein Diagramm, mit dessen Hilfe die Codes sortiert und zueinander in Beziehung gesetzt wurden. Zudem sind noch die Definitionen der einzelnen Codes angegeben. 


\section{A.1 Informed Consent}
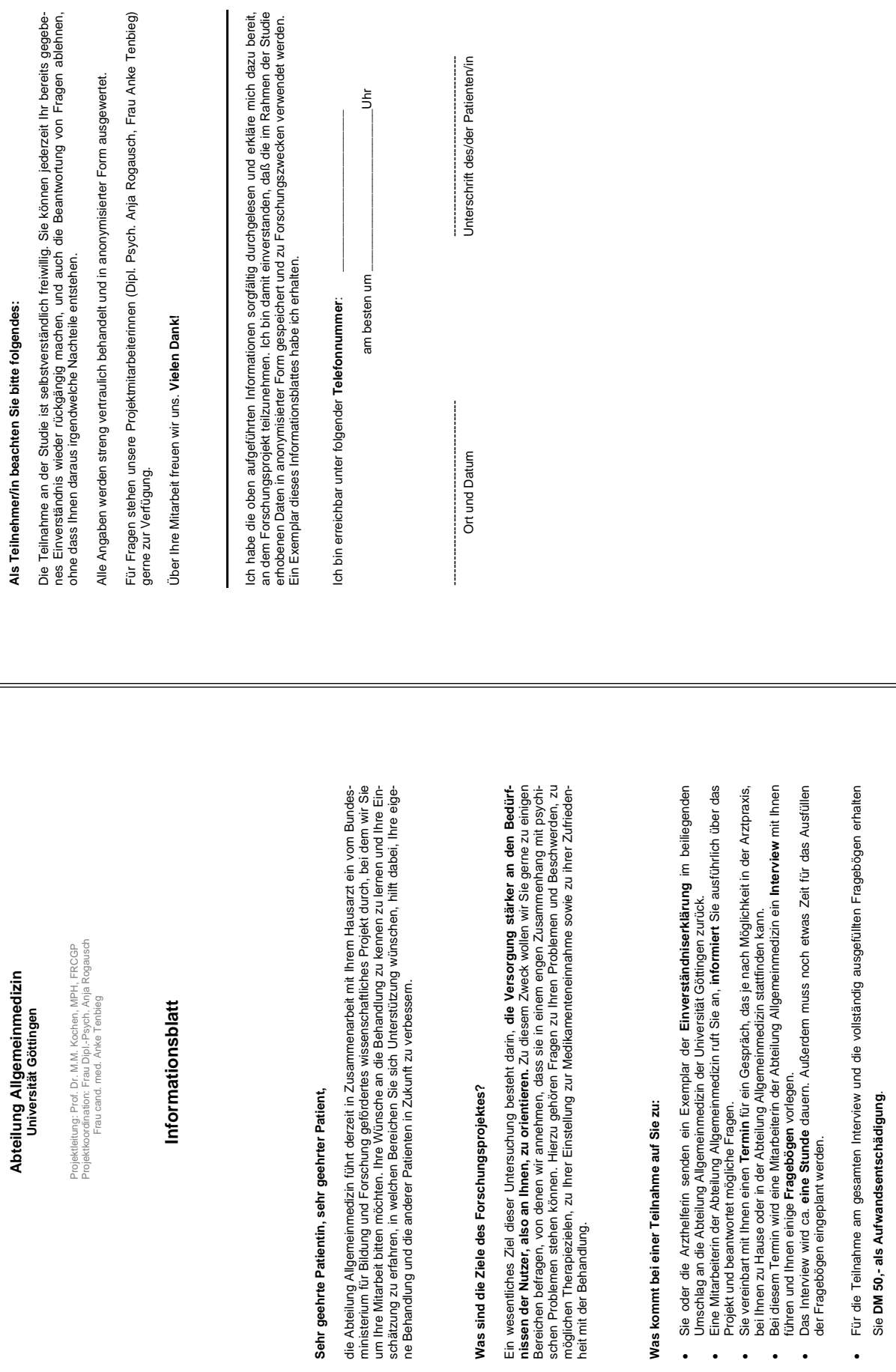


\section{A.2 Patientendaten}
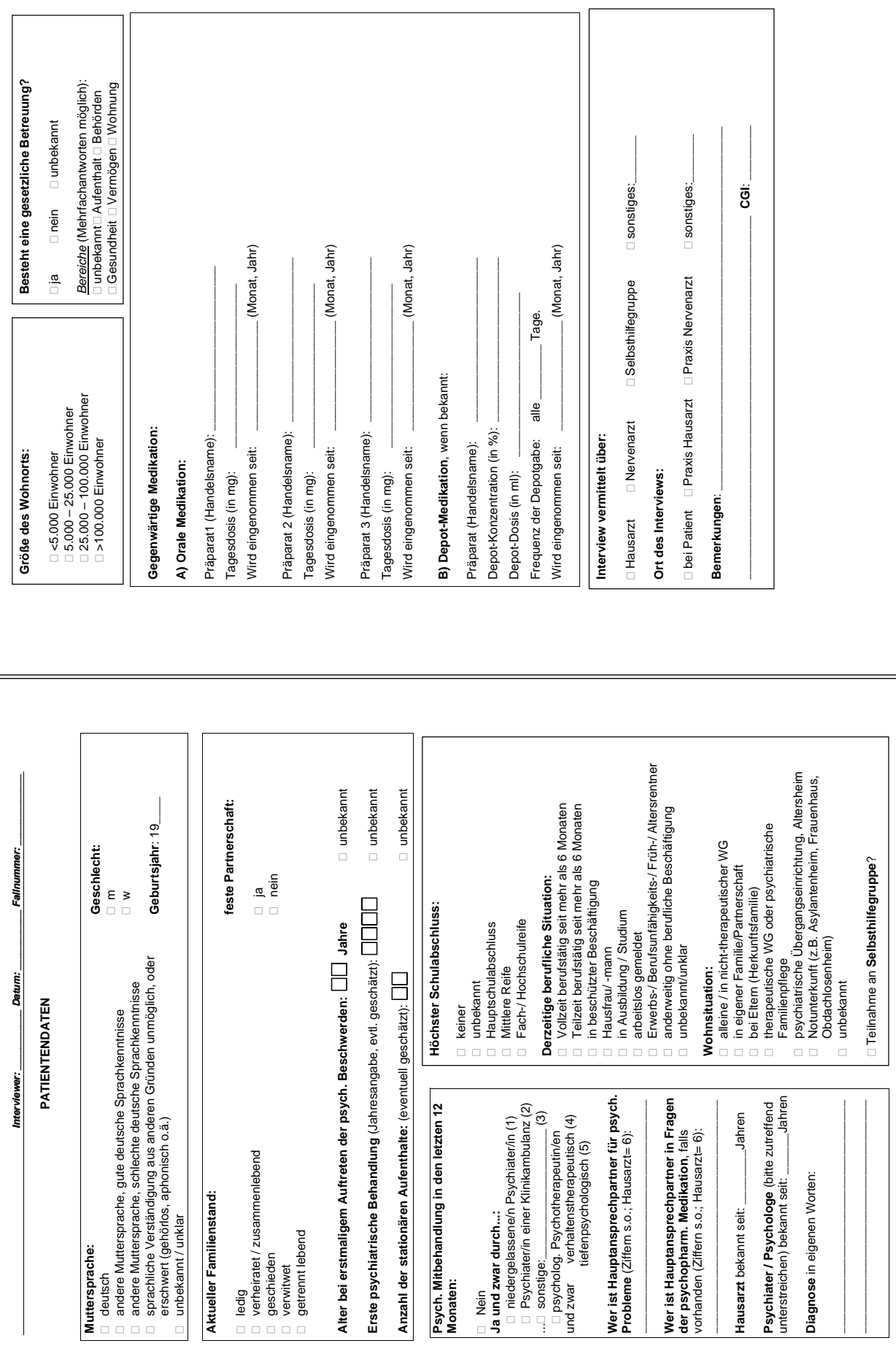


\section{A.3 Leitfaden Patienteninterview I}

\section{Ablauf der Interviews und Leitfaden}

Einleitung: Kurze Vorstellung, Erklären des Fragebogens, Danken für die Teilnahme

\section{Offener Beginn:}

Denken Sie bitte an das Verhalten Ihres Hausarztes, das Ihnen besonders positiv (negativ) aufgefallen ist. Beschreiben Sie die Situation und das Verhalten möglichst konkret.

- Beschreiben Sie das konkrete Verhalten des Arztes. Was fiel Ihnen daran besonders positiv (negativ) auf? Warum?

- Was waren die Konsequenzen dieses Verhaltens (z.B. auf ihr Befinden, ihre Zufriedenheit mit dem Arztbesuch)

\section{Betreuungssituation:}

Wer betreut Sie zur Zeit?

Wie lange schon?

Falls ein Arztwechsel stattgefunden hat: Warum?

Wie ist Patient genau an diesen Arzt gekommen?

Wie oft gehen sie zum jeweiligen Arzt?

\section{Arzt-Patient-Beziehung:}

Werden während des Besuchs auch persönliche Themen angesprochen? Können die eigenen Bedürfnisse geäußert werden?

Motivation: Zu welchem Arzt geht der Patient mit welchem Anliegen?

\section{Erwartungen:}

Was erwartet Patienten von einem Hausarzt?

Was erwartet Patienten von einem Psychiater?

\section{Zufriedenheit:}

Erfüllt der Hausarzt diese Erwartungen?

Erfüllt der Psychiater diese Erwartungen?

Was gefällt am Hausarzt besonders gut / besonders schlecht?

Was gefällt am Psychiater besonders gut / besonders schlecht? 


\section{A.4 Leitfaden Patienteninterview II}

\section{INTERVIEWLEITFADEN}

Stand 23.05.02

\section{Einstieg:}

o Ich möchte Interview führen und einige Daten notieren (Bogen „Patientendaten“). Schweigepflicht, Anonymität, Interview kann zugeschickt werden,

o Antworten werden nicht „interpretiert“, keine richtigen und falschen Antworten.

o Überweisung dauert ca. 8 Wochen.

In unserer Studie geht es ja um die Behandlung durch Hausärzte (HA) und andere Ärzte / Fachärzte (FA), z.B. Psychiater.

\section{Situation/ Krankheitsverlauf:}

o Könnten Sie mir einfach mal erzählen, um welche Störung es geht und welche Ärzte da eine Rolle spielten.

o Wie ist das bei Ihnen, gehen Sie in erster Linie zum Hausarzt oder gibt es auch einen Psychiater?

\section{Themenkomplex: Therapeutische Beziehung:}

o Wie würden Sie die Beziehung zu HA /FA beschreiben?

o Was macht für Sie eine gute Beziehung aus? Was muss ein Arzt (HA/FA) „mitbringen“ oder tun, damit Sie ihm vertrauen können?

o Fallen Ihnen auf Anhieb bestimmte Unterschiede zwischen dem Hausarzt und dem Psychiater ein? (z.B. Wenn Sie einem HA /FA gegenüber sitzen, wie fühlen Sie sich da, gibt es da gefühlsmäßig Unterschiede in Ihrem Erleben?)

o Mit welchem Anliegen gehen Sie zu welchem Arzt (HA/FA)? Worüber sprechen sie mit ihrem Arzt?

o Was sollte der Arzt (HA / FA) auf keinen Fall tun, wie sollte er sich auf keinen Fall verhalten? (Haben Sie schon einmal schlechte Erfahrungen gemacht?)

o Was würden Sie als Aufgaben des Arztes (HA/FA) im Umgang mit psychischen Störungen betrachten?

o Wer hat psychische Beschwerden erstmals erkannt und ernstgenommen? (Wer nicht, wie sind andere Ärzte damit umgegangen) 


\section{A.5 Codebook}

\section{Codes}

(überarbeitete Fassung vom 2.Feb.2003)

Determinanten der A-P-Beziehung

Länge der Beziehung

Kontakthäufigkeit

Kontaktzeit

Ideologische Übereinstimmung

Persönliche Beziehung

Autonomie

Informationsfluss

Kompetenzen und Zuständigkeiten

"Chemie"

Empathie

Erreichbarkeit

Soziales Umfeld

Fachliche Kompetenz

Gründe für (Non-)Compliance

$\mathrm{NC}$ Information

NC Erfahrungen

NC Nebenwirkungen

NC A-P-Beziehung

NC Einstellung

NC Logistik

Umgang mit Medikation innerhalb der therapeutischen Beziehung Ther. Bez. Medis

Umgang mit Non-Compliance innerhalb der therapeutischen Beziehung Ther. Bez. NC 


\section{A.6 Definitonen der Codes}
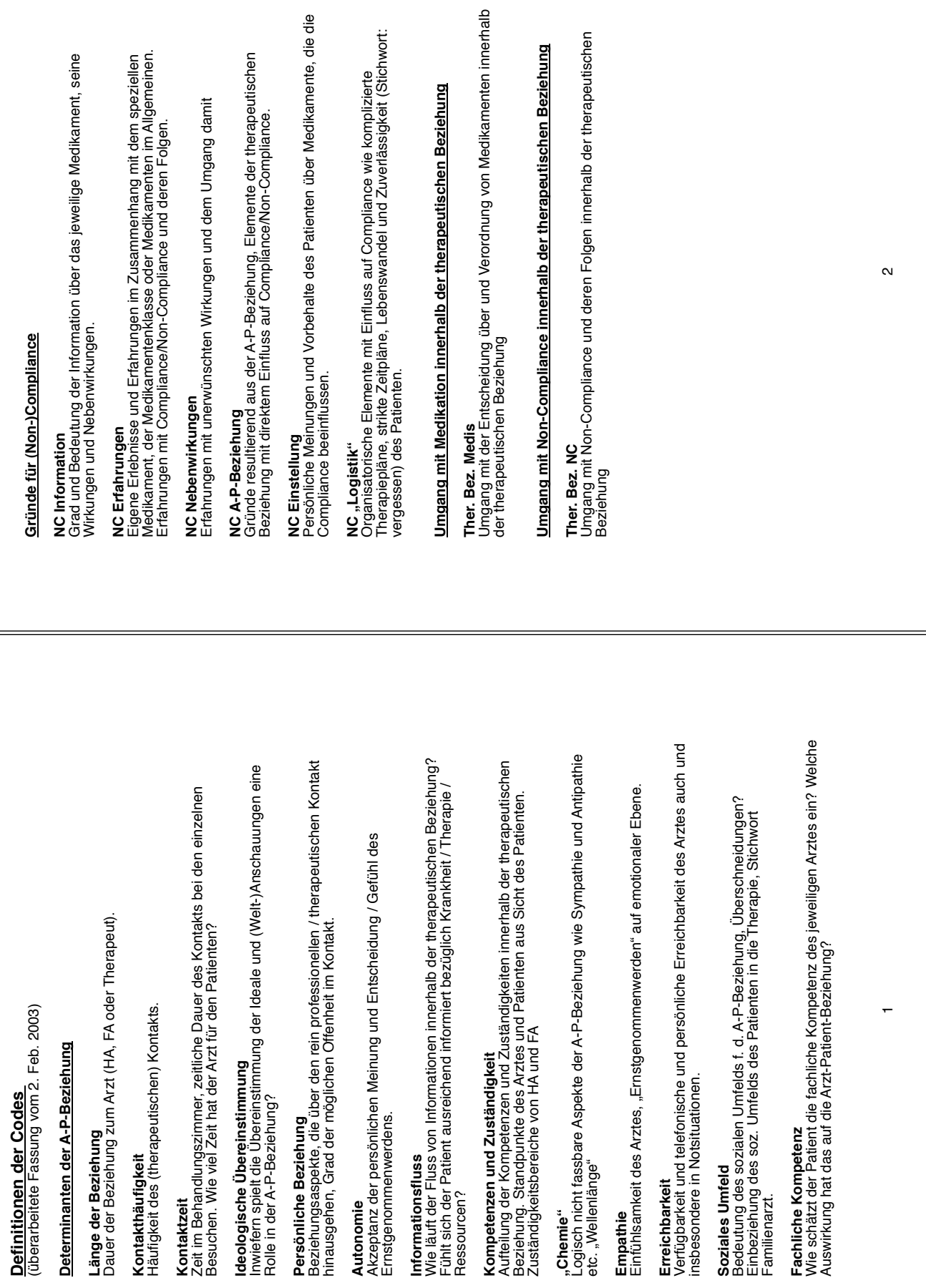


\section{A.7 Diagramm Codegruppen}

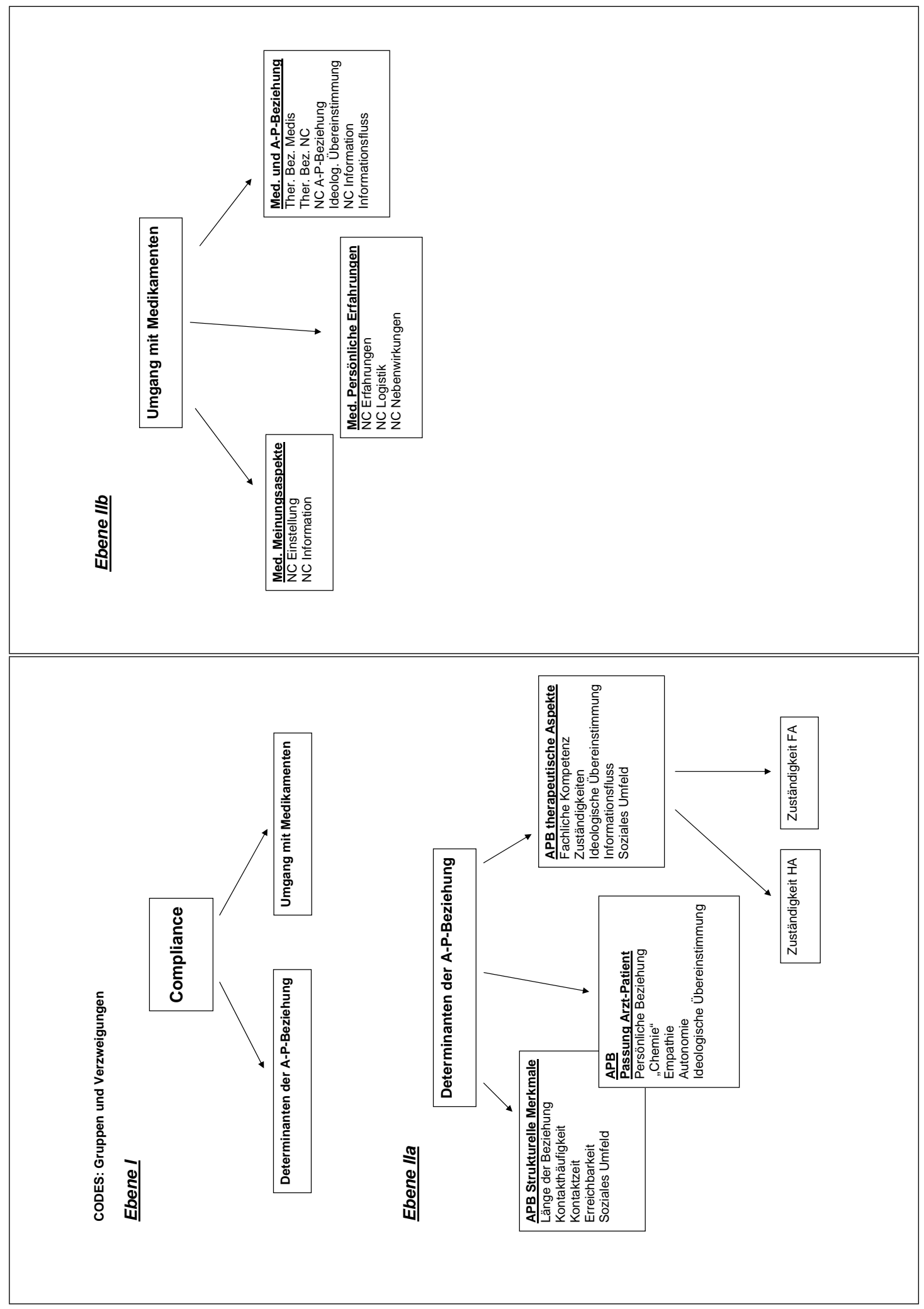




\section{Danksagung}

Ich möchte mich an dieser Stelle bei Prof. Dr. Wolfgang Himmel und Prof. Dr. Michael M. Kochem bedanken, die mir diese Dissertation im Rahmen des Projekts „Optimierung der Schizophreniebehandlung in der Allgemeinarztpraxis“ ermöglichten. Mein Dank gilt auch Anja Kapmeyer, MA und ganz besonders Dipl.-Psych. Anja Rogausch, die mich während der Arbeit inhaltlich, konzeptionell, praktisch und menschlich tatkräftig unterstützten.

Meinen herzlichen Dank möchte ich den Menschen ausdrücken, die sich zur Teilnahme an dem Projekt bereit erklärt haben, die ihre Türen geöffnet und ihre persönlichen Erfahrungen und Erlebnisse mitgeteilt haben. Ich habe diese Offenheit als Privileg empfunden.

Ich danke auch meiner Familie, die mich im Verlauf der Arbeit sowohl moralisch als auch finanziell unterstützt hat. Herzlichen Dank an Frank, der mir insbesondere in der Endphase im Kampf gegen die EDV unter die Arme gegriffen hat und meiner Frustration immer wieder Motivation entgegen setzen konnte. 


\section{Lebenslauf}

Ich wurde am 3.6.1976 als älteste Tochter von Hermann und Edith Tenbieg (geb. Schmidtke) in Hamburg geboren. Die Primarschulzeit absolvierte ich an der Grundschule Ellerau, der Overbergschule Oesede und der Orientierungsstufe Oesede. Es folgte der Wechsel an das Gymnasium Ursulaschule in Osnabrück. Im Rahmen eines Auslandsaufenthaltes in Vermilion, Ohio (USA) erwarb ich 1993 das amerikanische High School Diploma. Die deutsche Hochschulreife erlangte ich 1995 mit dem Abitur an der Ursulaschule. Nach einem initialen Studienbeginn der Fächer Life Science und Environmental Studies am Otterbein College in Westerville, Ohio (USA) wechselte ich 1997 in das Fach Humanmedizin. Hierfür kehrte ich nach Deutschland zurück und nahm im Sommersemester 1997 das Medizinstudium an der Georg-August-Universität Göttingen auf. Nach dem zweiten Teil der Ärztlichen Prüfung wechselte ich 2002 an die Eberhard-Karls-Universität Tübingen, wo ich im November 2003 das Medizinstudium mit dem dritten Teil der Ärztlichen Prüfung erfolgreich abschloss. Ab April 2004 war ich im Rahmen meiner Weiterbildung als Assistenzärztin in der Klinik für Chirurgie und Orthopädie des Kantonalen Spitals Grabs (Schweiz) tätig, von März 2007 bis Juni 2007 im selben Haus in der Klinik für Innere Medizin. Ab August 2007 werde ich meine Weiterbildung in der Klinik für Orthopädische Chirurgie des Kantonsspital St. Gallen (Schweiz) fortsetzen. 